Supporting Information

\title{
Visible-Light-Promoted Cross-Coupling of $N$-Alkylpyridinium Salts and Nitrostyrenes
}

Branislav Ferko, Michaela Marčeková, Katarína Ráchel Detková, Jana Doháňošová, Dušan Berkeš and Pavol Jakubec*

Faculty of Chemical and Food Technology, Slovak University of Technology, Radlinského 9, 81237 Bratislava, Slovakia

*e-mail: pavol.jakubec@stuba.sk 


\section{Content}

1. General experimental $\quad 4$

1.1. Reagents and solvents 4

1.2. Chromatography 4

1.3. Spectroscopy and instruments 4

1.4. Photoreactor set-up $\quad 4$

1.5. Starting materials 5

$\begin{array}{ll}\text { 2. Practical experimental } & 7\end{array}$

2.1. Synthesis and characterization of nitrostyrenes $\quad 7$

2.1.1. (E)-1-iodo-4-(2-nitrovinyl)benzene (1g) 7

2.1.2. (E)-2-bromo-5-(2-nitrovinyl)thiophene (1h) 7

2.1.3. (E)-4,4,5,5-tetramethyl-2-(4-(2-nitrovinyl)phenyl)-1,3,2-dioxaborolane (1i) 8

2.2. Synthesis and characterization of $N$-alkylpyridinium salts 9

2.2.1. Synthesis and characterization of $\mathbf{6 c} \quad 9$

2.2.2. Synthesis and characterization of $\mathbf{6 s} \quad 9$

2.3. Cross-coupling of $N$-alkylpyridinium salts and $\beta$-nitrostyrenes 10

2.3.1. Optimisation of the cross-coupling of $N$-alkylpyridinium salts and $B-\quad 10$ nitrostyrenes

2.3.2. Mechanistic study 12

2.3.3 General procedure for the cross-coupling of $N$-alkylpyridinium salts and $B-\quad 13$

nitrostyrenes

2.4. Synthesis and characterization of cross-coupling products 13

2.4.1. Synthesis and characterization of $\mathbf{3 f} \quad 13$

2.4.1.1. Synthesis of $\mathbf{3 f}$ on $1 \mathrm{mmol}$ scale 13

2.4.2. Synthesis and characterization of $\mathbf{3 g} \quad 14$

2.4.3. Synthesis and characterization of $\mathbf{3 h} \quad 14$

2.4.4. Synthesis and characterization of $\mathbf{3 i} \quad 14$

2.4.5. Synthesis and characterization of $\mathbf{3 j} \quad 15$

2.4.6. Synthesis and characterization of $\mathbf{3 k} \quad 15$

2.4.7. Synthesis and characterization of $\mathbf{3 l} \quad 15$

2.4.8. Synthesis and characterization of $\mathbf{3 m} \quad 16$

2.4.9. Synthesis and characterization of $\mathbf{3 n} \quad 16$

2.4.10. Synthesis and characterization of $\mathbf{3 o} \quad 16$

2.4.11. Synthesis and characterization of $\mathbf{3 p} \quad 17$

2.4.12. Synthesis and characterization of $\mathbf{3 q} \quad 17$

2.4.13. Synthesis and characterization of $\mathbf{3 r} \quad 17$

2.4.14. Synthesis and characterization of $3 \mathrm{~s} \quad 18$

2.4.15. Synthesis and characterization of $\mathbf{3 t} \quad 18$

2.4.16. Synthesis and characterization of $3 \mathbf{u} \quad 18$

2.4.17. Synthesis and characterization of $\mathbf{3 v} \quad 19$

2.4.18. Synthesis and characterization of $\mathbf{3 w} \quad 19$

2.4.19. Synthesis of $\mathbf{3 x} \quad 19$

2.4.20. Synthesis and characterization of $\mathbf{3 y} \quad 20$

2.4.21. Synthesis and characterization of $\mathbf{3 z} \quad 20$

2.4.22. Synthesis and characterization of 3aa 20

2.4.23. Synthesis and characterization of $\mathbf{3 a b} \quad 21$ 
2.4.24. Synthesis and characterization of 3ac

2.4.25. Synthesis and characterization of $\mathbf{3 a d}$

2.4.26. Synthesis and characterization of 3ae

2.4.27. Synthesis and characterization of $\mathbf{3 a f}$

2.4.28. Synthesis and characterization of $\mathbf{3 a g}$

2.4.29. Synthesis and characterization of 3ah

2.4.30. Synthesis and characterization of 3ai

2.4.31. Synthesis and characterization of 3aj 24

2.4.32. Synthesis and characterization of 3an 25

2.5. Limitations - Unsuccessful examples

4. ${ }^{1} \mathrm{H},{ }^{13} \mathrm{C}$, and ${ }^{19} \mathrm{~F}$ NMR spectra

4.1. ${ }^{1} \mathrm{H}$ and ${ }^{13} \mathrm{C}$ NMR spectra of $\mathbf{1 g} \quad 27$

4.2. ${ }^{1} \mathrm{H}$ and ${ }^{13} \mathrm{C}$ NMR spectra of $\mathbf{1 h}$

4.3. ${ }^{1} \mathrm{H}$ and ${ }^{13} \mathrm{C}$ NMR spectra of $\mathbf{1 i} \quad 29$

4.4. ${ }^{1} \mathrm{H},{ }^{13} \mathrm{C}$, and ${ }^{19} \mathrm{~F}$ NMR spectra of $\mathbf{6 c} \quad 30$

4.5. ${ }^{1} \mathrm{H},{ }^{13} \mathrm{C}$, and ${ }^{19} \mathrm{~F}$ NMR spectra of $6 \mathbf{s}$

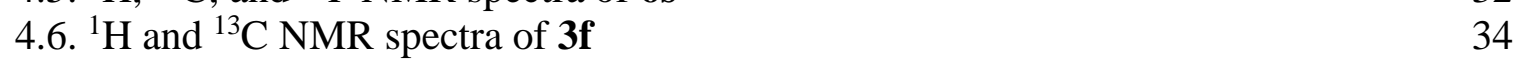

4.7. ${ }^{1} \mathrm{H}$ and ${ }^{13} \mathrm{C}$ NMR spectra of $\mathbf{3 g}$

4.8. ${ }^{1} \mathrm{H}$ and ${ }^{13} \mathrm{C}$ NMR spectra of $\mathbf{3 h}$

4.9. ${ }^{1} \mathrm{H}$ and ${ }^{13} \mathrm{C}$ NMR spectra of $\mathbf{3 i}$

4.10. ${ }^{1} \mathrm{H},{ }^{13} \mathrm{C}$, and ${ }^{19} \mathrm{~F}$ NMR spectra of $\mathbf{3 j} \quad 38$

4.11. ${ }^{1} \mathrm{H},{ }^{13} \mathrm{C}$, and ${ }^{19} \mathrm{~F}$ NMR spectra of $\mathbf{3 k} \quad 40$

4.12. ${ }^{1} \mathrm{H},{ }^{13} \mathrm{C}$, and ${ }^{19} \mathrm{~F}$ NMR spectra of $3 \mathrm{I}$

4.13. ${ }^{1} \mathrm{H}$ and ${ }^{13} \mathrm{C}$ NMR spectra of $3 \mathbf{m}$

4.14. ${ }^{1} \mathrm{H}$ and ${ }^{13} \mathrm{C}$ NMR spectra of $\mathbf{3 n} \quad 45$

4.15. ${ }^{1} \mathrm{H}$ and ${ }^{13} \mathrm{C}$ NMR spectra of 3o 46

4.16. ${ }^{1} \mathrm{H}$ and ${ }^{13} \mathrm{C}$ NMR spectra of $\mathbf{3 p} \quad 47$

4.17. ${ }^{1} \mathrm{H}$ and ${ }^{13} \mathrm{C}$ NMR spectra of $\mathbf{3 q} \quad 48$

4.18. ${ }^{1} \mathrm{H}$ and ${ }^{13} \mathrm{C}$ NMR spectra of $\mathbf{3 r}$

4.19. ${ }^{1} \mathrm{H}$ and ${ }^{13} \mathrm{C}$ NMR spectra of $3 \mathbf{s}$

4.20. ${ }^{1} \mathrm{H}$ and ${ }^{13} \mathrm{C}$ NMR spectra of $\mathbf{3 t}$

4.21. ${ }^{1} \mathrm{H}$ and ${ }^{13} \mathrm{C}$ NMR spectra of $\mathbf{3 u}$

4.22. ${ }^{1} \mathrm{H}$ and ${ }^{13} \mathrm{C}$ NMR spectra of $\mathbf{3 v}$

4.23. ${ }^{1} \mathrm{H}$ and ${ }^{13} \mathrm{C}$ NMR spectra of $\mathbf{3 w} \quad 54$

4.24. ${ }^{1} \mathrm{H}$ and ${ }^{13} \mathrm{C}$ NMR spectra of $\mathbf{3 y} \quad 55$

4.25. ${ }^{1} \mathrm{H}$ and ${ }^{13} \mathrm{C}$ NMR spectra of $\mathbf{3 z}$

4.26. ${ }^{1} \mathrm{H}$ and ${ }^{13} \mathrm{C}$ NMR spectra of 3aa

4.27. ${ }^{1} \mathrm{H}$ and ${ }^{13} \mathrm{C}$ NMR spectra of 3ab 58

4.28. ${ }^{1} \mathrm{H}$ and ${ }^{13} \mathrm{C}$ NMR spectra of 3ac $\quad 59$

4.29. ${ }^{1} \mathrm{H}$ and ${ }^{13} \mathrm{C}$ NMR spectra of $\mathbf{3 a d} \quad 60$

4.30. ${ }^{1} \mathrm{H}$ and ${ }^{13} \mathrm{C}$ NMR spectra of 3ae $\quad 61$

4.31. ${ }^{1} \mathrm{H}$ and ${ }^{13} \mathrm{C}$ NMR spectra of 3af 62

4.32. ${ }^{1} \mathrm{H}$ and ${ }^{13} \mathrm{C}$ NMR spectra of 3ag 63

4.33. ${ }^{1} \mathrm{H}$ and ${ }^{13} \mathrm{C}$ NMR spectra of 3ah 64

4.34. ${ }^{1} \mathrm{H}$ and ${ }^{13} \mathrm{C}$ NMR spectra of 3ai 65

4.35. ${ }^{1} \mathrm{H}$ and ${ }^{13} \mathrm{C}$ NMR spectra of 3aj 66

4.36. ${ }^{1} \mathrm{H}$ and ${ }^{13} \mathrm{C}$ NMR spectra of 3an 67 


\section{General experimental}

All reactions were performed without special precautions to avoid the presence of moisture unless otherwise stated.

\subsection{Reagents and solvents}

Reagents and solvents were obtained from commercial suppliers (Sigma Aldrich, Fluorochem, ABCR, Acros Organics) and used without further purification unless otherwise stated. Solvents were removed under reduced pressure using Heidolph and Büchi Rotavapor apparatus.

\subsection{Chromatography}

Column chromatography was carried out using Aldrich silica gel 60 (230-400 mesh, 40-63 $\mu \mathrm{m})$ and Merck Kieselgel $60(15-40 \mu \mathrm{m})$. All reactions were followed by thin-layer chromatography (TLC) where practical, using Merck Kieselgel 60 F254 (230-400 mesh) fluorescent treated silica, which was visualized under UV light $(254 \mathrm{~nm})$ or by staining with aqueous acidic ammonium molybdate or aqueous basic potassium permanganate solutions as appropriate. Where appropriate, the reaction progress was monitored by reverse-phase HPLC using E-COM machine set-up, Clarity DataApex software, and Nucleodur® Phenyl-Hexyl C18 column (Macherey-Nagel, $250 \times 4.0,5 \mu \mathrm{m}$ ). A gradient of water-acetonitrile-based eluents was used for most of the analyses.

\subsection{Spectroscopy and instruments}

All ${ }^{1} \mathrm{H},{ }^{13} \mathrm{C}$, and ${ }^{19} \mathrm{~F}$ NMR spectra were recorded using a Varian $600 \mathrm{MHz}$ or Varian $400 \mathrm{MHz}$ or $300 \mathrm{MHz}$ spectrometer. The chemical shift scales was calculated with the use of the resonance frequencies of TMS $\left(\delta_{\mathrm{C}}=\delta_{\mathrm{H}}=0.000 \mathrm{ppm}\right)$ and $\mathrm{CFCl}_{3}\left(\delta_{\mathrm{F}}=0.000 \mathrm{ppm}\right)$. Chemical shifts $(\delta)$ are given in parts per million (ppm), and coupling constants $(J)$ are given in Hertz $(\mathrm{Hz})$. The ${ }^{1} \mathrm{H}$ NMR spectra are reported as follows: $\delta / \mathrm{ppm}$ [number of protons, multiplicity (s $=$ singlet, $\mathrm{d}=$ doublet, $\mathrm{t}=$ triplet, $\mathrm{q}=$ quartet, quin $=$ quintet, $\mathrm{m}=$ multiplet, $" \mathrm{t} "=$ apparent triplet, "d" = apparent doublet, "q" = apparent quartet, $\mathrm{b}=$ broad), coupling constants $\mathrm{J} / \mathrm{Hz}$ (where appropriate)]. All NMR spectra and measurement parameters are available upon request from the corresponding author. High-resolution mass spectra (HRMS) were recorded on Orbitrap Velos PRO, Thermo Scientific machine.

\subsection{Photoreactor set-up}

Hepatochem EvoluChem ${ }^{\mathrm{TM}}$ PhotoRedOx photoreactor, equipped with EvoluChem ${ }^{\mathrm{TM}}$ LED 18W light (475 nm) or Kessil PR160L (467 nm, 100\% intensity) was used. An aluminum foil sheet cover was placed over the reactor during reactions. Photoreactor was equipped with the vial holder capable of carrying out up to 8 reactions at one time (using $4 \mathrm{ml}$ vials). Typically, the ambient temperature reached $26-32{ }^{\circ} \mathrm{C}$ during experiments with a fan on. The light source was placed 6-8 cm from the reaction vessel. The exact distance was dependent on the position in the vial holder. 


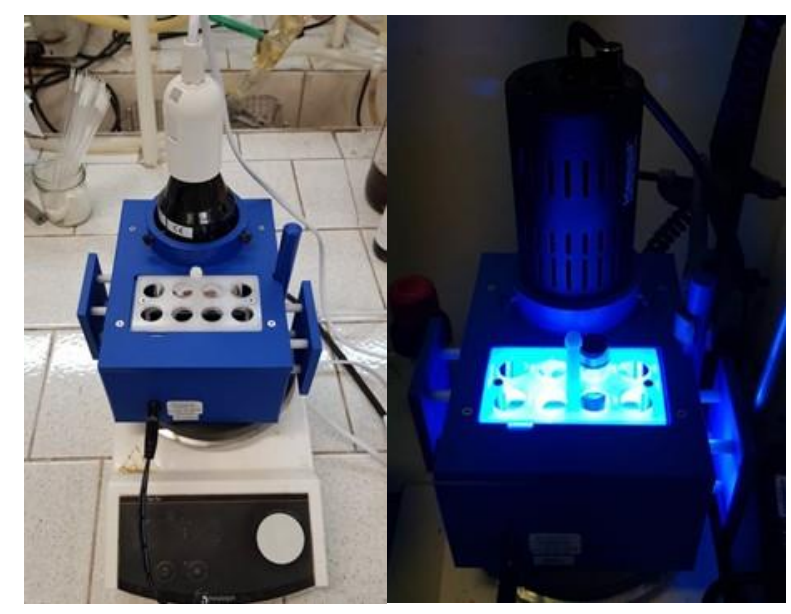

\subsection{Starting materials}

Starting materials, including 2,4,6-triphenylpyrylium tetrafluoroborate (18), amino acids (or corresponding hydrochloride salts and esters), photocatalysts (4CzIPN, [Ir $\left\{\mathrm{dFCF}_{3} \text { ppy }\right\}_{2}$ (bpy)] and Eosin Y), substituted benzaldehydes 17a-c, amines 19a,b and $\beta$-nitrostyrenes 1a-f, 1k, $\mathbf{1 m}, \mathbf{1 q}$ and $\mathbf{1 r}$ are commercially available from major worldwide suppliers. All other compounds were prepared either according to referenced literature procedures or newly developed procedures described in the text below.

\section{$\beta$-Nitrostyrenes:}
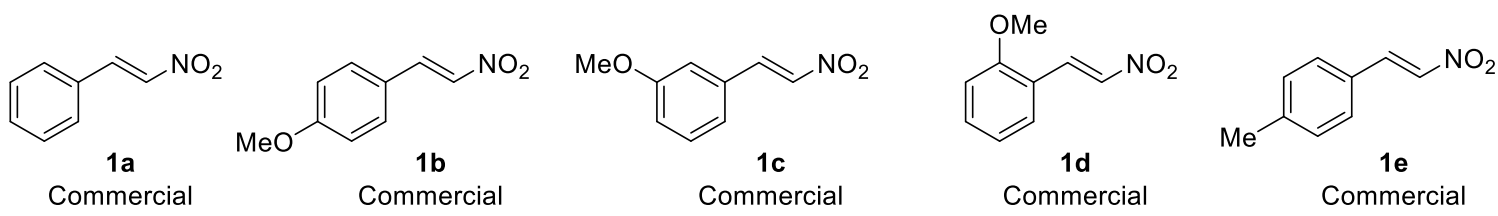
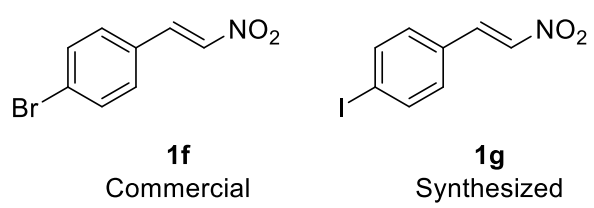

$1 \mathrm{~g}$

Synthesized<smiles>O=[N+]([O-])/C=C/c1cccc2ccccc12</smiles>

$1 j$

Synthesized

ref. 1<smiles>CCCC=C[N+](=O)[O-]</smiles>

10

Synthesized ref. 4

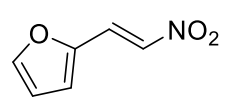

$1 \mathrm{k}$

Commercial<smiles>O=[N+]([O-])C=Cc1c[nH]c2ccccc12</smiles>

$1 p$

Synthesized

ref. 5

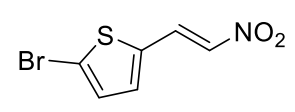

$1 \mathrm{~h}$

Synthesized<smiles>O=[N+]([O-])C=CC=Cc1ccccc1</smiles>

11

Synthesized ref. 2<smiles>C/C(=C\c1ccccc1)[N+](=O)[O-]</smiles>

$1 \mathrm{~m}$

Commercial<smiles>O=[N+]([O-])C=Cc1ccc([N+](=O)[O-])cc1</smiles>

19

Commercial

Synthesized 
N-Alkylpyridinium salts:<smiles></smiles>

6a,

Synthesized ref. 6<smiles></smiles>

$6 e$

Synthesized ref. 8<smiles></smiles>

6i

Synthesized ref. 10<smiles></smiles>

$6 \mathrm{~m}$

Synthesized ref. 9<smiles></smiles>

$6 q$ Synthesized ref. 7<smiles></smiles>

6u

Synthesized ref. 7

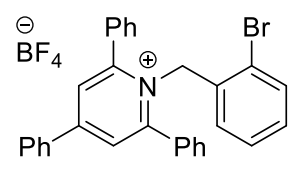

6b

Synthesized ref. 6<smiles></smiles>

$6 f$

Synthesized

ref. 6

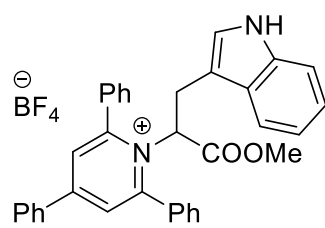

6j

Synthesized ref. 10<smiles></smiles>

$6 n$

Synthesized ref. 11

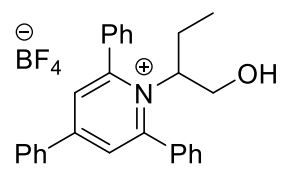

$6 r$

Synthesized

ref. 13

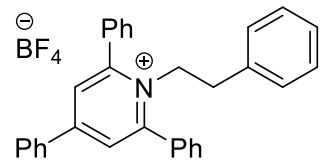

$6 v$

Synthesized ref. 11<smiles></smiles>

6c

Synthesized<smiles></smiles>

$6 \mathrm{~g}$

Synthesized ref. 7<smiles></smiles>

6k

Synthesized ref. 10<smiles></smiles>

60

Synthesized

ref. 12<smiles>CCCCCCCCCCCC[n+]1c(-c2ccccc2)cc(-c2ccccc2)cc1-c1ccccc1</smiles>

6s

Synthesized<smiles></smiles>

6w

Synthesized ref. 14

derived from mexiletine<smiles></smiles>

6d

Synthesized ref. 7<smiles></smiles>

$6 h$

Synthesized ref. 10<smiles></smiles>
6I

Synthesized ref. 10<smiles></smiles>

$6 p$

Synthesized ref. 11<smiles></smiles>

$6 t$

Synthesized

ref. 9 


\section{Practical experimental}

\subsection{Synthesis and characterization of $\beta$-nitrostyrenes}

\subsection{1. (E)-1-iodo-4-(2-nitrovinyl)benzene (1g)}

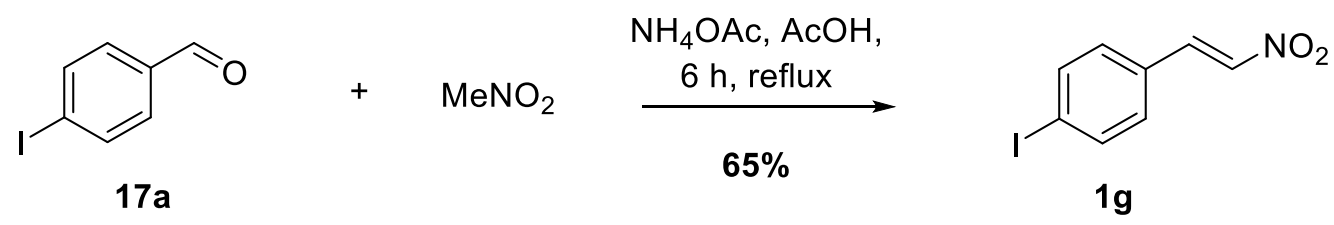

To a mixture of 4-iodobenzaldehyde $17 \mathbf{a}(21.55 \mathrm{mmol}, 5.000 \mathrm{~g})$ and nitromethane (3 equiv., $65 \mathrm{mmol}, 4.0 \mathrm{~g}, 3.5 \mathrm{ml}$ ) was added acetic acid $(16 \mathrm{ml})$ and ammonium acetate (1 equiv., $21.55 \mathrm{mmol}, 1.660 \mathrm{~g}$ ). The resulting mixture was stirred at reflux. After $6 \mathrm{~h}$, the mixture was cooled to $\mathrm{rt}$ and poured onto ice $(250 \mathrm{~g})$. The insoluble yellow solid was filtered off and recrystallized from a 1,4-dioxane- $\mathrm{MeOH}$ mixture $(35 \mathrm{ml}-150 \mathrm{ml})$, yielding nitroolefin $\mathbf{1 g}$ as a yellow crystalline solid $(3.86 \mathrm{~g}, 65 \%)$.

$\underline{{ }^{1} \mathbf{H} \text { NMR }}\left(600 \mathrm{MHz}, \mathrm{CDCl}_{3}\right) \delta 7.92(\mathrm{~d}, J=13.7 \mathrm{~Hz}, 1 \mathrm{H}), 7.82-7.79(\mathrm{~m}, 2 \mathrm{H}), 7.59(\mathrm{~d}, J=$ $13.7 \mathrm{~Hz}, 1 \mathrm{H}), 7.29-7.25(\mathrm{~m}, 2 \mathrm{H})$.

${ }^{13} \mathbf{C ~ N M R}\left(151 \mathrm{MHz}, \mathrm{CDCl}_{3}\right) \delta 138.9,138.1,137.7,130.5,129.6,99.2$.

$\underline{\text { HRMS }}(\mathrm{m} / \mathrm{z}):[\mathrm{M}+\mathrm{H}]^{+}$calcd for $\mathrm{C}_{8} \mathrm{H}_{7} \mathrm{INO}_{2}{ }^{+}, 275.9516$; found, 275.9515 .

Mp $182-184{ }^{\circ} \mathrm{C}$; lit. ${ }^{15} \mathrm{mp} 183-184{ }^{\circ} \mathrm{C}(\mathrm{EtOH})$.

\subsection{2. (E)-2-bromo-5-(2-nitrovinyl)thiophene (1h)}

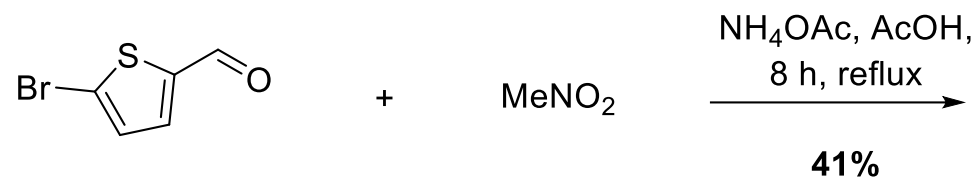

$17 \mathrm{~b}$<smiles>O=[N+]([O-])/C=C/c1ccc(Br)s1</smiles>

$1 \mathrm{~h}$

To a mixture of 4-bromothiophene-2-carbaldehyde $\mathbf{1 7 b}(30 \mathrm{mmol}, 5.7 \mathrm{~g}, 4.8 \mathrm{ml})$ and nitromethane (6.9 equiv., $207 \mathrm{mmol}, 12.6 \mathrm{~g}, 11.1 \mathrm{ml})$ was added acetic acid (36 ml) and ammonium acetate (2.4 equiv., $720 \mathrm{mmol}, 5.55 \mathrm{~g})$. The resulting mixture was stirred at reflux. After $8 \mathrm{~h}$, the mixture was cooled to $\mathrm{rt}$ and poured into water $(300 \mathrm{ml})$. The resulting mixture was extracted with EtOAc $(3 \times 50 \mathrm{ml})$, the combined organics were washed with brine $(50$ $\mathrm{ml})$, dried $\left(\mathrm{MgSO}_{4}\right)$, and concentrated in vacuo yielding brown oil. This residue was purified by column chromatography (hexane to hexanes-EtOAc 9:1), affording an orange solid (6.1 g). This material was recrystallized from hexane-EtOAc $(60 \mathrm{ml}-6 \mathrm{ml})$, yielding nitroolefin $\mathbf{1 h}$ as a yellow crystalline solid $(2.9 \mathrm{~g}, 41 \%)$.

$\underline{{ }^{1} \mathbf{H} \text { NMR }}\left(600 \mathrm{MHz}, \mathrm{CDCl}_{3}\right) \delta 8.02(\mathrm{~d}, J=13.4 \mathrm{~Hz}, 1 \mathrm{H}), 7.37(\mathrm{~d}, J=13.4 \mathrm{~Hz}, 1 \mathrm{H}), 7.20(\mathrm{~d}, J$ $=3.9 \mathrm{~Hz}, 1 \mathrm{H}), 7.11(\mathrm{~d}, J=3.9 \mathrm{~Hz}, 1 \mathrm{H})$.

${ }^{13} \mathbf{C ~ N M R}\left(151 \mathrm{MHz}, \mathrm{CDCl}_{3}\right) \delta 135.6,135.4,135.1,132.0,131.2,119.7$.

$\underline{\text { HRMS }}(\mathrm{m} / \mathrm{z}):[\mathrm{M}+\mathrm{H}]^{+}$calcd for $\mathrm{C}_{6} \mathrm{H}_{5} \mathrm{BrNO}_{2} \mathrm{~S}^{+}, 233.9219$; found, 233.9219.

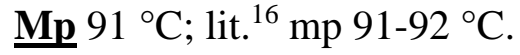




\subsection{3. (E)-4,4,5,5-tetramethyl-2-(4-(2-nitrovinyl)phenyl)-1,3,2-dioxaborolane (1i)}<smiles>CCO[N+](=O)[O-]</smiles>

To a mixture of 4-(4,4,5,5-tetramethyl-1,3,2-dioxaborolan-2-yl)benzaldehyde (17c) (9.26 mmol, $2.15 \mathrm{~g}$ ), nitromethane ( 1.1 equiv., $10.2 \mathrm{mmol}, 622 \mathrm{mg}, 547 \mu \mathrm{l})$ and $\mathrm{MeOH}(21.5 \mathrm{~mL})$ was added sodium hydroxide $(10.2 \mathrm{mmol}, 408 \mathrm{mg})$ at $0{ }^{\circ} \mathrm{C}$ and the resulting mixture was stirred at this temperature for 2 hours. After $2 \mathrm{~h}$ (approximately 50\% conversion to adduct was achieved), the mixture was poured into $3 \mathrm{M} \mathrm{HCl}(20 \mathrm{ml})$ and diluted with diethyl ether (50 $\mathrm{ml})$. The aqueous phase was separated and reextracted with diethyl ether $(2 \times 25 \mathrm{ml})$. The combined organics were washed with brine $(50 \mathrm{ml})$, dried $\left(\mathrm{MgSO}_{4}\right)$, and concentrated in vacuo yielding crude oil (2.26 g), which was used for the next step without further purification. This residue was dissolved in DCM $(39 \mathrm{ml})$ and cooled to $0{ }^{\circ} \mathrm{C}$. Triethylamine $(2$ equiv., $15 \mathrm{mmol}, 1.6 \mathrm{~g}, 2.1 \mathrm{ml}$ ) was added, followed by methane sulfonyl chloride (1 equiv., $7.71 \mathrm{mmol}, 883 \mathrm{mg}, 597 \mu \mathrm{l}$ ) and the resulting mixture was stirred at rt. After $12 \mathrm{~h}$, the mixture was diluted with brine $(40 \mathrm{ml})$, the aqueous phase was separated and reextracted with $\mathrm{CH}_{2} \mathrm{Cl}_{2}$ $(2 \times 20 \mathrm{ml})$, the combined organics were dried $\left(\mathrm{MgSO}_{4}\right)$ and concentrated in vacuo yielding crude oil, which after crystallization from ethanol gave nitroolefin $\mathbf{1 i}$ as a pale-yellow crystalline solid (384 mg, $18 \%$ ).

Note: The isolated nitroolefin $1 \mathbf{i}$ contains approximately $9 \%$ of starting material-aldehyde $17 \mathbf{c}$ (See attached NMR).

$\underline{{ }^{1} \mathbf{H ~ N M R}}\left(600 \mathrm{MHz}, \mathrm{CDCl}_{3}\right) \delta 7.99(\mathrm{~d}, J=13.7 \mathrm{~Hz}, 1 \mathrm{H}), 7.87(\mathrm{~d}, J=8.0 \mathrm{~Hz}, 2 \mathrm{H}), 7.61(\mathrm{~d}, J$ $=13.7 \mathrm{~Hz}, 1 \mathrm{H}), 7.53(\mathrm{~d}, J=8.0 \mathrm{~Hz}, 2 \mathrm{H}), 1.35(\mathrm{~s}, 12 \mathrm{H})$.

$\underline{{ }^{13} \mathbf{C ~ N M R}}\left(151 \mathrm{MHz}, \mathrm{CDCl}_{3}\right) \delta 139.0,137.8,135.7,132.5,128.3,84.4,25.0$.

$\underline{\text { HRMS }}(\mathrm{m} / z):[\mathrm{M}+\mathrm{H}]^{+}$calcd for $\mathrm{C}_{14} \mathrm{H}_{19} \mathrm{BNO}_{4}{ }^{+}, 276.1402$; found, 276.1400 . 


\subsection{Synthesis and characterization of $N$-alkylpyridinium salts}<smiles>[P-]#[P+]c1cc(-c2ccccc2)cc(-c2ccccc2)[o+]1</smiles>

18

\subsubsection{Synthesis and characterization of $6 c$}<smiles>NCc1cccc(Br)c1</smiles>

$19 a$

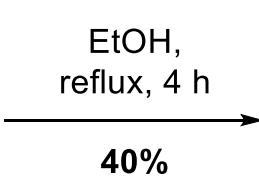<smiles></smiles>

$6 \mathrm{c}$

To a mixture of pyrylium salt $18(10.1 \mathrm{mmol}, 4.00 \mathrm{~g})$ and amine 19a (1.2 equiv., $12.1 \mathrm{mmol}$, $2.25 \mathrm{~g}, 1.52 \mathrm{ml})$ was added dry EtOH $(16 \mathrm{ml})$ under argon atmosphere, and the resulting mixture was stirred at reflux. After $4 \mathrm{~h}$, the mixture was cooled to $\mathrm{rt}$, and diethyl ether $(50 \mathrm{ml})$ was added. The precipitated solid was filtered off, washed with diethyl ether, and recrystallized from EtOH, affording $\mathbf{6 c}(2.26 \mathrm{~g}, 40 \%)$ as a white solid.

${ }^{1} \mathbf{H}$ NMR $\left(600 \mathrm{MHz}, \mathrm{CDCl}_{3}\right) \delta 7.97(\mathrm{~s}, 2 \mathrm{H}), 7.82(\mathrm{~m}, 2 \mathrm{H}), 7.68-7.63(\mathrm{~m}, 4 \mathrm{H}), 7.62-7.57$ $(\mathrm{m}, 1 \mathrm{H}), 7.57-7.47(\mathrm{~m}, 8 \mathrm{H}), 7.30(\mathrm{~d}, J=8.8 \mathrm{~Hz}, 1 \mathrm{H}), 6.99(\mathrm{t}, J=7.9 \mathrm{~Hz}, 1 \mathrm{H}), 6.53(\mathrm{~s}, 1 \mathrm{H})$, $6.46(\mathrm{~d}, J=7.8 \mathrm{~Hz}, 1 \mathrm{H}), 5.75(\mathrm{~s}, 2 \mathrm{H})$.

${ }^{13} \mathbf{C ~ N M R}\left(151 \mathrm{MHz}, \mathrm{CDCl}_{3}\right) \delta 157.6,156.7,136.1,133.7,132.7,132.6,131.6,131.3,130.6$, $129.9,129.79,129.4,129.2,128.3,126.7,125.2,122.8,57.6$.

19 $\mathbf{F}$ NMR $\left(564 \mathrm{MHz}, \mathrm{CDCl}_{3}\right) \delta-152.72$ (minor), -152.78 (major).

HRMS $(m / z):[\mathrm{M}+\mathrm{H}]^{+}$calcd for $\mathrm{C}_{30} \mathrm{H}_{23} \mathrm{BrN}^{+}$, 476.1008; found, 476.1006.

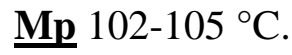

\subsubsection{Synthesis and characterization of $6 \mathrm{~s}$}<smiles>[P-]#[P+]c1cc(-c2ccccc2)cc(-c2ccccc2)[o+]1</smiles>

18<smiles>CCCCCCCCCCCCN</smiles>

19b

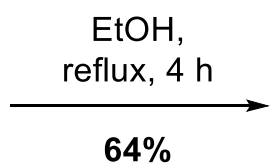

$64 \%$<smiles></smiles>

6s

To a mixture of pyrylium salt 18 (3.00 mmol, $1.19 \mathrm{~g})$ and lauryl amine $\mathbf{1 9 b}$ (1.1 equiv., 3.30 mmol, $612 \mathrm{mg}$ ) was added dry EtOH $(6 \mathrm{ml})$ under argon atmosphere, and the resulting mixture (deep red suspension) was stirred at reflux. After $4 \mathrm{~h}$, the mixture (deep red solution) was cooled to rt and concentrated under reduced pressure affording orange-red oil. To this residue, MTBE (30 ml) was added, and the resulting mixture (orange solution) was left standing in the freezer $\left(-20^{\circ} \mathrm{C}\right)$. After $24 \mathrm{~h}$, the precipitated solid was filtered off and washed with MTBE $\left(10 \mathrm{ml}\right.$, precooled to $\left.-20^{\circ} \mathrm{C}\right)$, yielding $\mathbf{6 s}(1.08 \mathrm{~g}, 64 \%)$ as a white solid.

${ }^{1} \mathbf{H}$ NMR $\left(600 \mathrm{MHz}, \mathrm{CDCl}_{3}\right) \delta 7.88-7.72(\mathrm{~m}, 8 \mathrm{H}), 7.63-7.47(\mathrm{~m}, 9 \mathrm{H}), 4.42-4.37(\mathrm{~m}, 2 \mathrm{H})$, $1.46-1.38(\mathrm{~m}, 2 \mathrm{H}), 1.32-1.14(\mathrm{~m}, 9 \mathrm{H}), 1.14-1.06(\mathrm{~m}, 2 \mathrm{H}), 1.01(\mathrm{~m}, 2 \mathrm{H}), 0.93-0.84(\mathrm{~m}$, $5 \mathrm{H}), 0.74(\mathrm{~s}, 3 \mathrm{H})$.

13C NMR $\left(151 \mathrm{MHz}, \mathrm{CDCl}_{3}\right) \delta 156.6,155.8,134.2,132.9,132.1,131.1,129.8,129.8,129.4$, 129.2, 128.2, 126.8, 54.9, 32.0, 29.8, 29.7, 29.67, 29.6, 29.5, 29.4, 29.35, 29.0, 28.0, 26.1, $22.8,14.2$.

${ }^{19}$ F NMR $\left(564 \mathrm{MHz}, \mathrm{CDCl}_{3}\right) \delta-153.28$ (minor), -153.34 (major).

HRMS $(\mathrm{m} / z):[\mathrm{M}+\mathrm{H}]^{+}$calcd for $\mathrm{C}_{35} \mathrm{H}_{42} \mathrm{~N}^{+}, 476.3312$; found, 476.3309 .

Mp $58-61^{\circ} \mathrm{C}$. 


\subsection{Cross-coupling of $N$-alkylpyridinium salts and B-nitrostyrenes}

\subsubsection{Optimization of the cross-coupling of $N$-alkylpyridinium salts and B-nitrostyrenes}

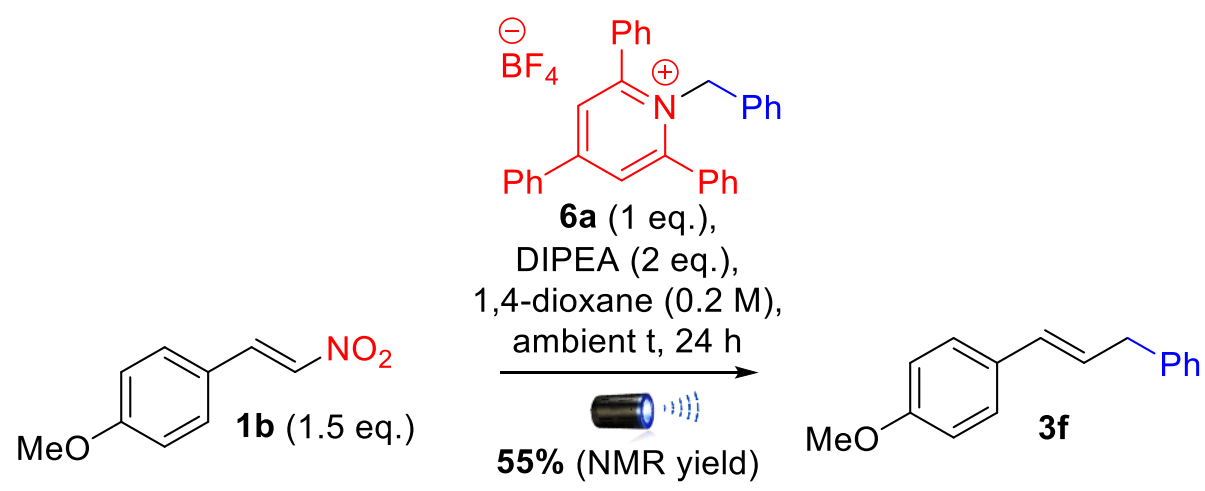

\begin{tabular}{|c|c|c|}
\hline Entry & Change from standard conditions & Yield $\mathbf{3 f} \mathbf{f}^{\mathrm{a}}$ \\
\hline 1 & None & $55 \%(43 \%)$ \\
\hline 2 & 4CzIPN (5 mol \%) added & $50 \%$ \\
\hline 3 & Eosin $\mathrm{Y}(5 \mathrm{~mol} \%)$ added & $41 \%$ \\
\hline 4 & $\begin{array}{l}{\left[\operatorname{Ir}\left\{\mathrm{dFCF}_{3} p p y\right\}_{2}(\mathrm{bpy})\right] \quad\left(\begin{array}{lll}5 & \mathrm{~mol} & \%\end{array}\right)} \\
\text { added }\end{array}$ & $16 \%$ \\
\hline 5 & $\mathrm{Et}_{3} \mathrm{~N}$ instead of DIPEA & $45 \%$ \\
\hline 6 & TMEDA instead of DIPEA & $51 \%$ \\
\hline 7 & $\mathrm{MeCN}$ instead of 1,4-dioxane & $32 \%$ \\
\hline 8 & EtOAc instead of 1,4-dioxane & $51 \%$ \\
\hline 9 & DCE instead of 1,4-dioxane & $47 \%$ \\
\hline 10 & $\mathrm{CHCl}_{3}$ instead of 1,4-dioxane & $27 \%$ \\
\hline 11 & $\mathrm{CH}_{2} \mathrm{Cl}_{2}$ instead of 1,4-dioxane & $47 \%$ \\
\hline 12 & Without blue light in dark & $0 \%$ \\
\hline 13 & Without DIPEA & $0 \%$ \\
\hline 14 & Performed under argon & $55 \%$ \\
\hline 15 & $\mathbf{1 b}$ (1 eq.) and $\mathbf{6 a}$ (1.5 eq.), $20 \mathrm{~h}$ & $31 \%$ \\
\hline 16 & Hantzsch ester instead of DIPEA & $0 \%$ \\
\hline 17 & Hantzsch ester ( 2 eq.) added & $52 \%$ \\
\hline
\end{tabular}

${ }^{\text {a }}$ determined by ${ }^{1} \mathrm{H}$ NMR analysis against an internal standard $\left(\mathrm{ClCH}_{2} \mathrm{CHCl}_{2}\right)$, the yield in brackets is isolated. All reactions were performed on $0.4 \mathrm{mmol}$ scale. 


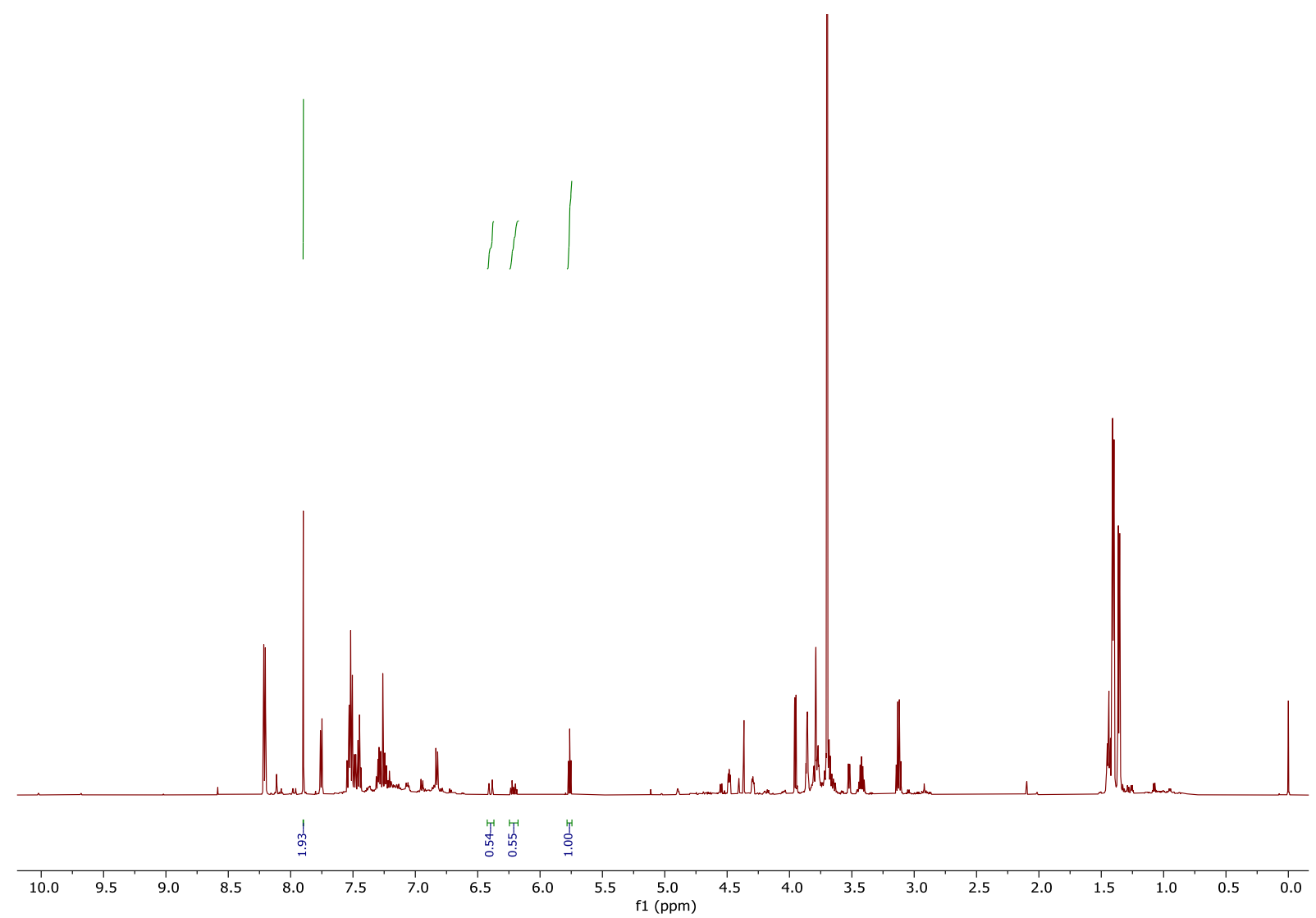

${ }^{1} \mathrm{H}$ NMR spectrum of the crude reaction mixture with internal standard, $\mathrm{ClCH}_{2} \mathrm{CHCl}_{2}$ (entry 1). 


\subsubsection{Mechanistic study}

A, Formation of dimer $\mathbf{7}$ under the standard conditions

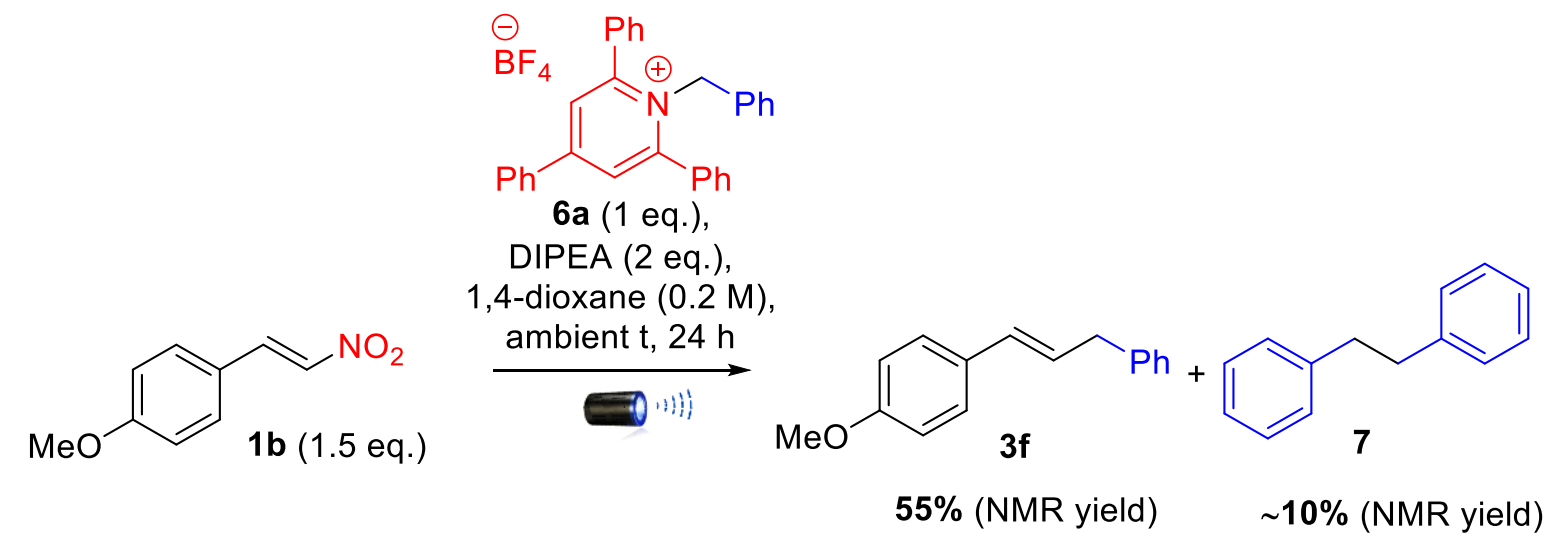

$\mathrm{B}$, Addition of TEMPO to the reaction mixture

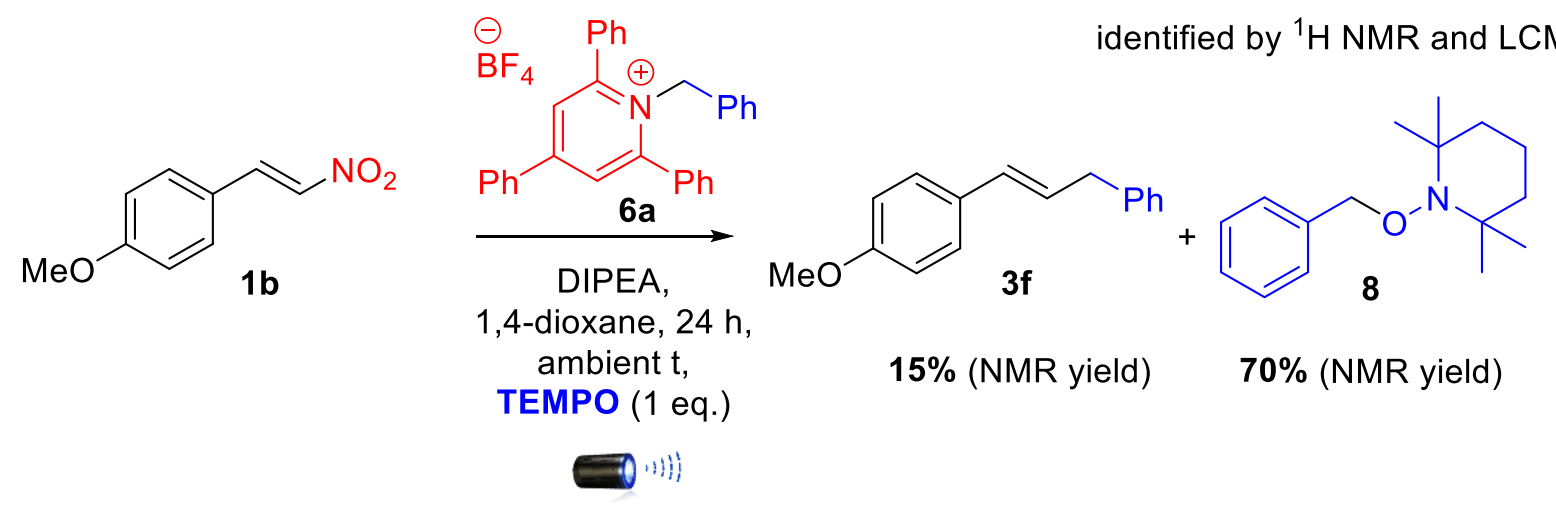

C, Reaction without nitrostyrene

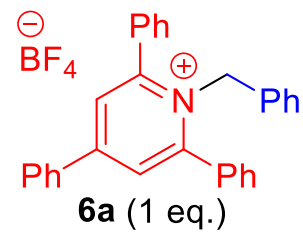

DIPEA (2 eq.),

1,4-dioxane (0.2 M),

No nitrostyrene
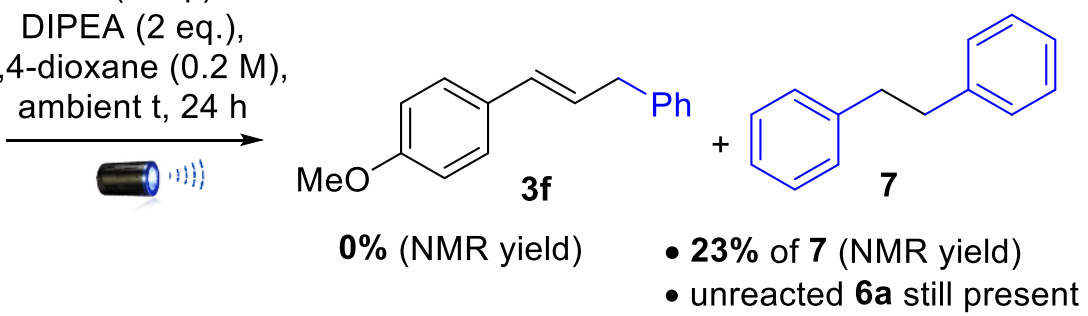

Redox potentials:

- $N$-Alkylpyridinium salt $6 \mathbf{a} \mathrm{E}=-0.92 \mathrm{~V}$ vs. $\mathrm{SCE}^{17,18}$

- DIPEA $_{\text {ox }}=0.94 \mathrm{~V}$ vs. SCE ${ }^{19}$ 


\subsubsection{General procedure for the cross-coupling of $N$-alkylpyridinium salts and B- nitrostyrenes}

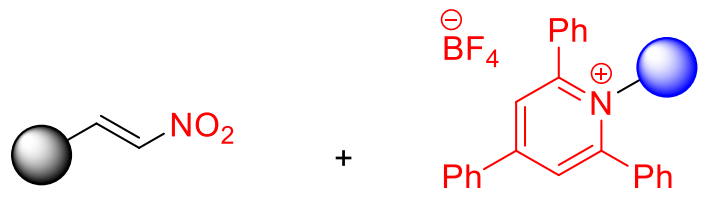

1
6
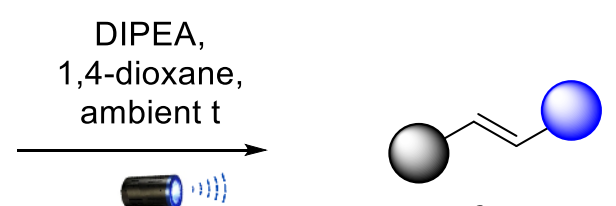

3

A $4 \mathrm{ml}$ vial equipped with a magnetic stirring bar was charged with pyridinium salt 6 (1 equiv., 0.2 or $0.4 \mathrm{mmol}$ ) and appropriate nitrostyrene 1 (1.5 equiv.). 1,4-Dioxane $(0.2 \mathrm{M}$ or $0.1 \mathrm{M}$ concentration of $\mathbf{6}$ ) and DIPEA ( 2 equiv.) were added. After the addition, the vial was sealed, wrapped with parafilm, and then irradiated with blue light while being stirred (500 rpm). The progress of the reaction was monitored by TLC and HPLC. The specific reaction time is indicated in each experiment. After completing the reaction, the volatiles were removed under reduced pressure, and the crude product was purified by column chromatography.

Note: All isolated compounds $\mathbf{3}$ possess $E$-configuration of the double bond affected during the cross-coupling. The configuration was determined from the size of the coupling constant of the $\mathrm{C}-\mathrm{C}$ double bond.

\subsection{Synthesis and characterization of cross-coupling products}

\subsubsection{Synthesis and characterization of $3 f$}

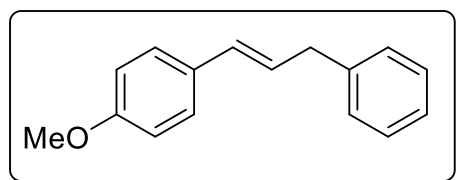

Compound $3 f$ was synthesized according to the General procedure using pyridinium salt 6a $(0.400 \mathrm{mmol}, 194 \mathrm{mg})$, nitrostyrene $\mathbf{1 b}(0.600 \mathrm{mmol}, 108 \mathrm{mg})$ in 1,4 -dioxane $(2 \mathrm{ml}, 0.2$ $\mathrm{M})$ and irradiated with blue light for $16 \mathrm{~h}$. The crude mixture was purified by silica gel chromatography (hexanes- $\left.\mathrm{CH}_{2} \mathrm{Cl}_{2} 9: 1\right)$ to give $\mathbf{3 f}(38.8 \mathrm{mg}, 43 \%)$ as a white solid.

${ }_{6}^{1} \mathbf{H}$ NMR $\left(600 \mathrm{MHz}, \mathrm{CDCl}_{3}\right) \delta 7.32-7.27(\mathrm{~m}, 4 \mathrm{H}), 7.26-7.19(\mathrm{~m}, 3 \mathrm{H}), 6.85-6.81(\mathrm{~m}, 2 \mathrm{H})$, $6.40(\mathrm{~d}, J=15.7 \mathrm{~Hz}, 1 \mathrm{H}), 6.21(\mathrm{dt}, J=15.7,7.0 \mathrm{~Hz}, 1 \mathrm{H}), 3.79(\mathrm{~s}, 3 \mathrm{H}), 3.53(\mathrm{~d}, J=7.0 \mathrm{~Hz}$, $2 \mathrm{H})$.

${ }_{13}^{13}$ NMR $\left(151 \mathrm{MHz}, \mathrm{CDCl}_{3}\right) \delta 159.0,140.6,130.6,130.5,128.8,128.6,127.4,127.2,126.2$, 114.1, 55.4, 39.5 .

$\underline{\text { HRMS }}(\mathrm{m} / \mathrm{z}):[\mathrm{M}+\mathrm{H}]^{+}$calcd for $\mathrm{C}_{16} \mathrm{H}_{17} \mathrm{O}^{+}, 225.1274$; found, 225.1274 .

All data are in good agreement with previously published data. ${ }^{20}$

\subsubsection{Synthesis of $3 f$ on $1 \mathrm{mmol}$ scale}

Compound 3f was synthesized according to the General procedure using pyridinium salt $6 \mathbf{6}$ (1.00 mmol, $485 \mathrm{mg})$, nitrostyrene 1 b $(1.50 \mathrm{mmol}, 269 \mathrm{mg})$ and DIPEA (2 eq., $2.00 \mathrm{mmol}$, $258 \mathrm{mg}, 348 \mu \mathrm{L})$ in 1,4-dioxane $(5 \mathrm{ml}, 0.2 \mathrm{M})$ and irradiated with blue light for $16 \mathrm{~h}$. The reaction was performed in a $25 \mathrm{~mL}$ round bottom flask equiped with a stirrer bar and rubber septum located inside the photoreactor. The crude mixture (55\% NMR yield) was purified by silica gel chromatography (hexane to hexanes- $\mathrm{CH}_{2} \mathrm{Cl}_{2}$ 9:1) to give $\mathbf{3 f}(92 \mathrm{mg}, 41 \%)$ as a white solid. The diferrence in yields for $0.4 \mathrm{mmol}(43 \%)$ and $1 \mathrm{mmol}(41 \%)$ experiments was attributed to an acceptable experimental error. 


\subsubsection{Synthesis and characterization of $3 \mathrm{~g}$}

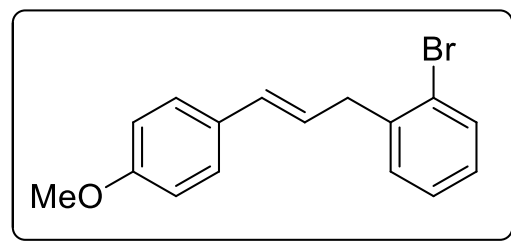

Compound 3g was synthesized according to the General procedure using pyridinium salt $\mathbf{6 b}(0.400 \mathrm{mmol}, 226 \mathrm{mg})$, nitrostyrene $\mathbf{1 b}(0.600 \mathrm{mmol}, 108 \mathrm{mg})$ in 1,4-dioxane $(2 \mathrm{ml}$, $0.2 \mathrm{M}$ ), and irradiated with blue light for $24 \mathrm{~h}$. The crude mixture was purified by silica gel chromatography (gradient: hexanes- $\left.\mathrm{CH}_{2} \mathrm{Cl}_{2}\right)$ to give $\mathbf{3 g}(52 \mathrm{mg}, 43 \%)$ as a yellow oil.

${ }_{1}^{1} \mathbf{H}$ NMR $\left(600 \mathrm{MHz}, \mathrm{CDCl}_{3}\right) \delta 7.55(\mathrm{dd}, J=8.0,1.0 \mathrm{~Hz}, 1 \mathrm{H}), 7.31-7.22(\mathrm{~m}, 4 \mathrm{H}), 7.10-$ $7.05(\mathrm{~m}, 1 \mathrm{H}), 6.85-6.80(\mathrm{~m}, 2 \mathrm{H}), 6.40(\mathrm{~d}, J=15.8 \mathrm{~Hz}, 1 \mathrm{H}), 6.19(\mathrm{dt}, J=15.8,6.8 \mathrm{~Hz}, 1 \mathrm{H})$, $3.79(\mathrm{~s}, 3 \mathrm{H}), 3.63(\mathrm{dd}, J=6.8,1.2 \mathrm{~Hz}, 2 \mathrm{H})$.

${ }^{13} \mathbf{C ~ N M R}\left(151 \mathrm{MHz}, \mathrm{CDCl}_{3}\right) \delta 159.1,140.0,132.9,131.3,130.6,130.4,128.0,127.7,127.4$, 125.3, 124.7, 114.1, 55.4, 39.5.

$\underline{\text { HRMS }}(\mathrm{m} / \mathrm{z}):[\mathrm{M}+\mathrm{H}]^{+}$calcd for $\mathrm{C}_{16} \mathrm{H}_{16} \mathrm{BrO}^{+}, 303.0379$; found, 303.0378 .

All data are in good agreement with previously published data. ${ }^{20}$

\subsubsection{Synthesis and characterization of $3 \mathrm{~h}$}

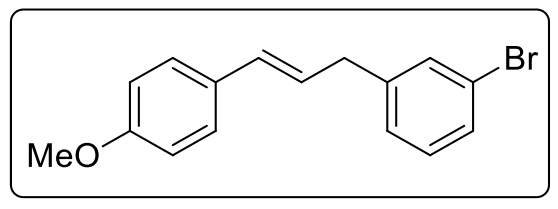

Compound 3h was synthesized according to the General procedure using pyridinium salt $\mathbf{6 c}(0.400 \mathrm{mmol}, 226 \mathrm{mg})$, nitrostyrene $\mathbf{1 b}(0.600 \mathrm{mmol}, 108 \mathrm{mg})$ in 1,4-dioxane $(2$ $\mathrm{ml}, 0.2 \mathrm{M}$ ), and irradiated with blue light for $24 \mathrm{~h}$. The crude mixture was purified by silica gel chromatography (gradient: hexanes- $\mathrm{CH}_{2} \mathrm{Cl}_{2}$ ) to give $\mathbf{3 h}(43 \mathrm{mg}, 36 \%$ ) as a yellow oil.

${ }_{1} \mathbf{H}$ NMR $\left(600 \mathrm{MHz}, \mathrm{CDCl}_{3}\right) \delta 7.40(\mathrm{bs}, 1 \mathrm{H}), 7.37-7.33(\mathrm{~m}, 1 \mathrm{H}), 7.32-7.29(\mathrm{~m}, 2 \mathrm{H}), 7.20-$ $7.15(\mathrm{~m}, 2 \mathrm{H}), 6.87-6.83(\mathrm{~m}, 2 \mathrm{H}), 6.41(\mathrm{~d}, J=15.7 \mathrm{~Hz}, 1 \mathrm{H}), 6.17(\mathrm{dt}, J=15.7,7.0 \mathrm{~Hz}, 1 \mathrm{H})$, $3.81(\mathrm{~s}, 3 \mathrm{H}), 3.50(\mathrm{dd}, J=7.0,0.9 \mathrm{~Hz}, 2 \mathrm{H})$.

${ }^{13} \mathbf{C ~ N M R}\left(151 \mathrm{MHz}, \mathrm{CDCl}_{3}\right) \delta 159.2,143.0,131.8,131.3,130.2,130.1,129.4,127.4,126.1$, 122.7, 114.1, 55.4, 39.1.

All data are in good agreement with previously published data. ${ }^{20}$

\subsubsection{Synthesis and characterization of $3 i$}

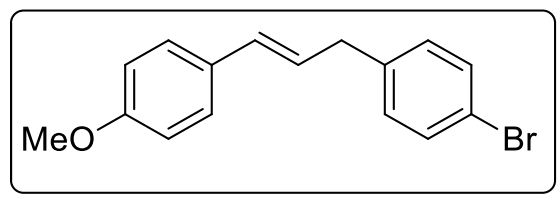

Compound 3i was synthesized according to the General procedure using pyridinium salt $\mathbf{6 d}(0.400 \mathrm{mmol}, 226$ $\mathrm{mg})$, nitrostyrene $\mathbf{1 b}(0.600 \mathrm{mmol}, 108 \mathrm{mg})$ in 1,4-dioxane $(2 \mathrm{ml}, 0.2 \mathrm{M})$, and irradiated with blue light for $24 \mathrm{~h}$. The crude mixture was purified by silica gel chromatography (gradient: hexanes- $\mathrm{CH}_{2} \mathrm{Cl}_{2}$ ) to give $\mathbf{3 i}(44 \mathrm{mg}, 38 \%$ ) as a yellow solid.

$\underline{{ }^{1} \mathbf{H ~ N M R}}\left(600 \mathrm{MHz}, \mathrm{CDCl}_{3}\right) \delta 7.44-7.38(\mathrm{~m}, 2 \mathrm{H}), 7.30-7.25(\mathrm{~m}, 2 \mathrm{H}), 7.14-7.06(\mathrm{~m}, 2 \mathrm{H})$, $6.87-6.81(\mathrm{~m}, 2 \mathrm{H}), 6.37(\mathrm{~d}, J=15.7 \mathrm{~Hz}, 1 \mathrm{H}), 6.15(\mathrm{dt}, J=15.7,6.9 \mathrm{~Hz}, 1 \mathrm{H}), 3.79(\mathrm{~s}, 3 \mathrm{H})$, $3.46(\mathrm{~d}, J=6.9 \mathrm{~Hz}, 2 \mathrm{H})$.

${ }_{113}{ }^{13}$ NMR $\left(151 \mathrm{MHz}, \mathrm{CDCl}_{3}\right) \delta 159.1,139.5,131.6,131.1,130.6,130.2,127.4,126.4,120.0$, 114.1, 55.4, 38.8 .

$\underline{\text { HRMS }}(\mathrm{m} / \mathrm{z}):[\mathrm{M}+\mathrm{H}]^{+}$calcd for $\mathrm{C}_{16} \mathrm{H}_{16} \mathrm{BrO}^{+}, 303.0379$; found, 303.0379.

$\overline{\text { All data }}$ are in good agreement with previously published data. ${ }^{20}$ 


\subsubsection{Synthesis and characterization of $3 \mathbf{j}$}

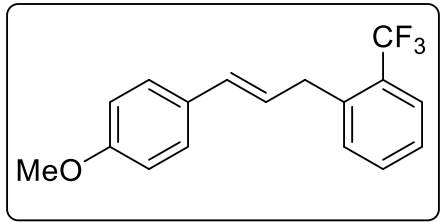

Compound $\mathbf{3 j}$ was synthesized according to the General procedure using pyridinium salt 6e $(0.400 \mathrm{mmol}, 221 \mathrm{mg})$, nitrostyrene $\mathbf{1 b}(0.600 \mathrm{mmol}, 108 \mathrm{mg})$ in 1,4-dioxane $(2 \mathrm{ml}, 0.2$ $\mathrm{M})$ and irradiated with blue light for $24 \mathrm{~h}$. The crude mixture was purified by silica gel chromatography (gradient: hexanes$\mathrm{CH}_{2} \mathrm{Cl}_{2}$ ) to give $\mathbf{3 j}(29 \mathrm{mg}, 25 \%)$ as a yellow oil.

1 H NMR $\left(600 \mathrm{MHz}, \mathrm{CDCl}_{3}\right) \delta 7.65(\mathrm{~d}, J=7.8 \mathrm{~Hz}, 1 \mathrm{H}), 7.48(\mathrm{t}, J=7.5 \mathrm{~Hz}, 1 \mathrm{H}), 7.41(\mathrm{~d}, J=$ $7.7 \mathrm{~Hz}, 1 \mathrm{H}), 7.35-7.28(\mathrm{~m}, 3 \mathrm{H}), 6.87-6.81(\mathrm{~m}, 2 \mathrm{H}), 6.42(\mathrm{~d}, J=15.8 \mathrm{~Hz}, 1 \mathrm{H}), 6.19$ (dt, $J=$ $15.7,6.9 \mathrm{~Hz}, 1 \mathrm{H}), 3.80(\mathrm{~s}, 3 \mathrm{H}), 3.71(\mathrm{~d}, J=6.9 \mathrm{~Hz}, 2 \mathrm{H})$.

${ }^{13} \mathbf{C ~ N M R}\left(151 \mathrm{MHz}, \mathrm{CDCl}_{3}\right) \delta 159.1,139.3,132.0,131.4,131.4,130.2,129.6$ - $127.8(\mathrm{~m})$, 127.5, 126.3, $126.1-126.0(\mathrm{~m}), 124.7$ (q, $J=274 \mathrm{~Hz}), 114.1,55.4,35.9$ (q, $J=4 \mathrm{~Hz})$.

${ }^{19}$ F NMR $\left(564 \mathrm{MHz}, \mathrm{CDCl}_{3}\right) \delta-59.8$.

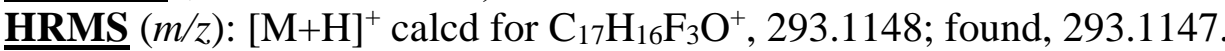

All data are in good agreement with previously published data. ${ }^{21}$

\subsubsection{Synthesis and characterization of $3 \mathrm{k}$}

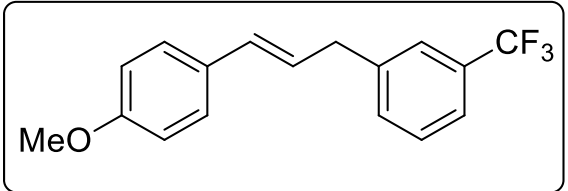

Compound 3k was synthesized according to the General procedure using pyridinium salt $6 \mathbf{f}(0.400 \mathrm{mmol}, 221$ $\mathrm{mg})$, nitrostyrene $\mathbf{1 b}(0.600 \mathrm{mmol}, 108 \mathrm{mg})$ in 1,4dioxane $(2 \mathrm{ml}, 0.2 \mathrm{M})$, and irradiated with blue light for $24 \mathrm{~h}$. The crude mixture was purified by silica gel chromatography (gradient: hexanes- $\mathrm{CH}_{2} \mathrm{Cl}_{2}$ ) to give $\mathbf{3 k}(46 \mathrm{mg}, 39 \%)$ as a yellow oil.

$\underline{{ }^{1} \mathbf{H ~ N M R}}\left(600 \mathrm{MHz}, \mathrm{CDCl}_{3}\right) \delta 7.51-7.40(\mathrm{~m}, 4 \mathrm{H}), 7.32-7.28(\mathrm{~m}, 2 \mathrm{H}), 6.87-6.83(\mathrm{~m}, 2 \mathrm{H})$, $6.43(\mathrm{~d}, J=15.7 \mathrm{~Hz}, 1 \mathrm{H}), 6.19(\mathrm{dt}, J=15.6,7.0 \mathrm{~Hz}, 1 \mathrm{H}), 3.81(\mathrm{~s}, 3 \mathrm{H}), 3.58(\mathrm{~d}, J=7.0 \mathrm{~Hz}$, $2 \mathrm{H})$.

${ }^{13} \mathbf{C ~ N M R}\left(151 \mathrm{MHz}, \mathrm{CDCl}_{3}\right) \delta 159.2,141.6,132.2(\mathrm{q}, J=1 \mathrm{~Hz}), 131.5,130.9(\mathrm{q}, J=31 \mathrm{~Hz})$, $130.1,129.0,127.5,125.9,125.5(\mathrm{q}, J=5 \mathrm{~Hz}), 124.4(\mathrm{q}, J=270 \mathrm{~Hz}), 123.2(\mathrm{q}, J=5 \mathrm{~Hz})$, $114.14,55.4,39.2$.

${ }^{19} \mathbf{F ~ N M R}\left(564 \mathrm{MHz}, \mathrm{CDCl}_{3}\right) \delta-62.6$.

$\underline{\text { HRMS }}(\mathrm{m} / \mathrm{z}):[\mathrm{M}+\mathrm{H}]^{+}$calcd for $\mathrm{C}_{17} \mathrm{H}_{16} \mathrm{~F}_{3} \mathrm{O}^{+}, 293.1148$; found, 293.1147 .

\subsubsection{Synthesis and characterization of 31}

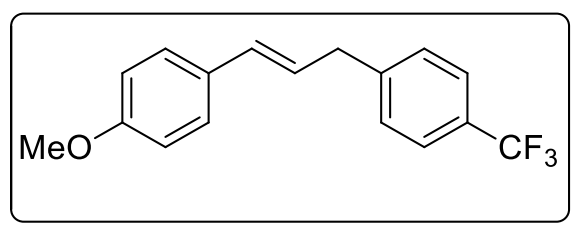

Compound 31 was synthesized according to the General procedure using pyridinium salt $\mathbf{6 g}(0.400 \mathrm{mmol}, 221$ $\mathrm{mg})$, nitrostyrene $\mathbf{1 b}(0.600 \mathrm{mmol}, 108 \mathrm{mg})$ in $1,4-$ dioxane $(2 \mathrm{ml}, 0.2 \mathrm{M})$, and irradiated with blue light for $24 \mathrm{~h}$. The crude mixture was purified by silica gel chromatography (gradient: hexanes- $\left.\mathrm{CH}_{2} \mathrm{Cl}_{2}\right)$ to give $\mathbf{3 l}(36 \mathrm{mg}, 31 \%)$ as a yellow oil.

1H NMR $\left(300 \mathrm{MHz}, \mathrm{CDCl}_{3}\right) \delta 7.56(\mathrm{~d}, J=8.0 \mathrm{~Hz}, 2 \mathrm{H}), 7.37-7.33(\mathrm{~m}, 2 \mathrm{H}), 7.32-7.26(\mathrm{~m}$, $2 \mathrm{H}), 6.87-6.81(\mathrm{~m}, 2 \mathrm{H}), 6.42(\mathrm{~d}, J=15.7 \mathrm{~Hz}, 1 \mathrm{H}), 6.17(\mathrm{dt}, J=15.7,6.9 \mathrm{~Hz}, 1 \mathrm{H}), 3.80(\mathrm{~s}$, $3 \mathrm{H}), 3.58(\mathrm{~d}, J=6.9 \mathrm{~Hz}, 2 \mathrm{H})$.

${ }^{13} \mathbf{C ~ N M R}\left(151 \mathrm{MHz}, \mathrm{CDCl}_{3}\right) \delta 159.2,144.7$ (broad), 131.5, 130.1, 129.1, 128.66 (q, $J=34$ $\mathrm{Hz}), 127.4,125.9,125.5(\mathrm{q}, J=5 \mathrm{~Hz}), 124.5(\mathrm{q}, J=270 \mathrm{~Hz}), 114.13,55.4,39.2$.

${ }^{19}$ F NMR $\left(564 \mathrm{MHz}, \mathrm{CDCl}_{3}\right) \delta-62.4$.

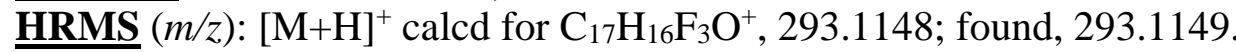

All data are in good agreement with previously published data. ${ }^{21}$ 


\subsubsection{Synthesis and characterization of $3 \mathrm{~m}$}

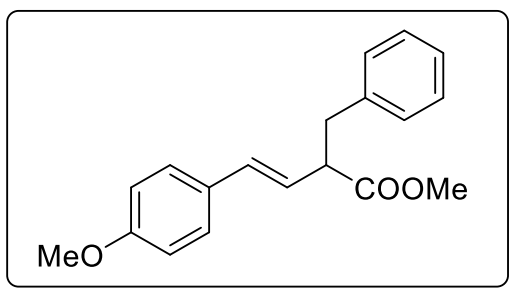

Compound $\mathbf{3 m}$ was synthesized according to General procedure using pyridinium salt $\mathbf{6 h}(0.400 \mathrm{mmol}, 223 \mathrm{mg})$, nitrostyrene $\mathbf{1 b}(0.600 \mathrm{mmol}, 108 \mathrm{mg})$ in 1,4 -dioxane $(2 \mathrm{ml}$, $0.2 \mathrm{M})$, and irradiated with blue light for $16 \mathrm{~h}$. The crude mixture was purified by silica gel chromatography (gradient: hexanes- $\left.\mathrm{CH}_{2} \mathrm{Cl}_{2}\right)$ to give $\mathbf{3 m}(49.3 \mathrm{mg}, 42 \%)$ as a yellow solid.

$\underline{{ }^{1} \mathbf{H ~ N M R}}\left(600 \mathrm{MHz}, \mathrm{CDCl}_{3}\right) \delta 7.27-7.24(\mathrm{~m}, 4 \mathrm{H}), 7.21-7.15(\mathrm{~m}, 3 \mathrm{H}), 6.84-6.79(\mathrm{~m}, 2 \mathrm{H})$, $6.35(\mathrm{~d}, J=15.8 \mathrm{~Hz}, 1 \mathrm{H}), 6.08(\mathrm{dd}, J=15.8,8.8 \mathrm{~Hz}, 1 \mathrm{H}), 3.79(\mathrm{~s}, 3 \mathrm{H}), 3.63(\mathrm{~s}, 3 \mathrm{H}), 3.47-$ $3.43(\mathrm{~m}, 1 \mathrm{H}), 3.15$ (dd, $J=13.7,8.0 \mathrm{~Hz}, 1 \mathrm{H}), 2.92$ (dd, $J=13.7,7.0 \mathrm{~Hz}, 1 \mathrm{H})$.

${ }^{13} \mathbf{C ~ N M R}\left(151 \mathrm{MHz}, \mathrm{CDCl}_{3}\right) \delta 174.1,159.4,138.8,132.2,129.6,129.2,128.5,127.6,126.5$, 124.7, 114.1, 55.4, 52.0, 51.5, 39.2.

$\underline{\text { HRMS }}(\mathrm{m} / z):[\mathrm{M}+\mathrm{H}]^{+}$calcd for $\mathrm{C}_{19} \mathrm{H}_{21} \mathrm{O}_{3}{ }^{+}, 297.1485$; found, 297.1485.

\subsubsection{Synthesis and characterization of $3 n$}

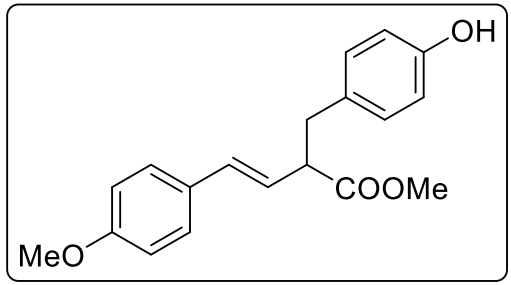

Compound 3n was synthesized according to the General procedure using pyridinium salt $\mathbf{6 i}(0.400 \mathrm{mmol}, 229 \mathrm{mg})$, nitrostyrene $\mathbf{1 b}(0.600 \mathrm{mmol}, 108 \mathrm{mg})$ in 1,4-dioxane $(2 \mathrm{ml}$, $0.2 \mathrm{M}$ ), and irradiated with blue light for $16 \mathrm{~h}$. The crude mixture was purified by silica gel chromatography (gradient: hexanes- $\left.\mathrm{CH}_{2} \mathrm{Cl}_{2}\right)$ to give $3 \mathrm{n}(64.6 \mathrm{mg}, 52 \%)$ as a yellow solid.

${ }^{1} \mathbf{H}$ NMR $\left(600 \mathrm{MHz}, \mathrm{CDCl}_{3}\right) \delta 7.29-7.24(\mathrm{~m}, 2 \mathrm{H}), 7.07-7.01(\mathrm{~m}, 2 \mathrm{H}), 6.85-6.81(\mathrm{~m}, 2 \mathrm{H})$, $6.74-6.69(\mathrm{~m}, 2 \mathrm{H}), 6.34(\mathrm{~d}, J=15.8 \mathrm{~Hz}, 1 \mathrm{H}), 6.06(\mathrm{dd}, J=15.8,8.8 \mathrm{~Hz}, 1 \mathrm{H}), 4.65(\mathrm{bs}, 1 \mathrm{H})$, $3.80(\mathrm{~s}, 3 \mathrm{H}), 3.64(\mathrm{~s}, 3 \mathrm{H}), 3.41-3.37(\mathrm{~m}, 1 \mathrm{H}), 3.08(\mathrm{dd}, J=13.8,8.0 \mathrm{~Hz}, 1 \mathrm{H}), 2.85(\mathrm{dd}, J=$ $13.8,7.0 \mathrm{~Hz}, 1 \mathrm{H})$.

${ }^{13} \mathbf{C}$ NMR $\left(151 \mathrm{MHz}, \mathrm{CDCl}_{3}\right) \delta 174.5,159.4,154.4,132.2,130.8,130.3,129.7,127.7,124.7$, 115.4, 114.1, 55.4, 52.1, 51.9, 38.4.

HRMS $(\mathrm{m} / z):[\mathrm{M}+\mathrm{H}]^{+}$calcd for $\mathrm{C}_{19} \mathrm{H}_{21} \mathrm{O}_{4}{ }^{+}, 313.1434$; found, 313.1433 .

\subsubsection{Synthesis and characterization of 30}

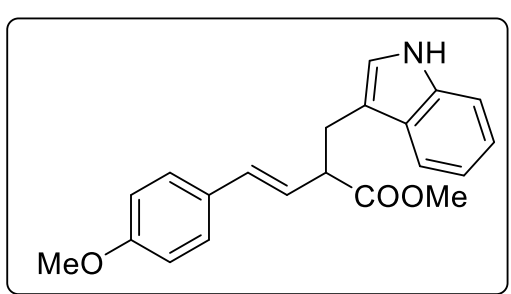

Compound 3o was synthesized according to the General procedure using pyridinium salt $\mathbf{6 j}(0.400 \mathrm{mmol}, 239 \mathrm{mg})$, nitrostyrene $\mathbf{1 b}(0.600 \mathrm{mmol}, 108 \mathrm{mg})$ in 1,4-dioxane $(2 \mathrm{ml}$, $0.2 \mathrm{M})$, and irradiated with blue light for $16 \mathrm{~h}$. The crude mixture was purified by silica gel chromatography (gradient: hexanes- $\mathrm{CH}_{2} \mathrm{Cl}_{2}$ ) and then (hexanes-EtOAc 4:1) to give 30 $(58.5 \mathrm{mg}, 44 \%)$ as an orange oil.

$\underline{{ }^{1} \mathbf{H} \text { NMR }}\left(600 \mathrm{MHz}, \mathrm{CDCl}_{3}\right) \delta 7.94(\mathrm{bs}, 1 \mathrm{H}), 7.62(\mathrm{~d}, J=7.9 \mathrm{~Hz}, 1 \mathrm{H}), 7.35(\mathrm{~d}, J=8.1 \mathrm{~Hz}$, $1 \mathrm{H}), 7.29-7.25(\mathrm{~m}, 2 \mathrm{H}), 7.21-7.17(\mathrm{~m}, 1 \mathrm{H}), 7.15-7.10(\mathrm{~m}, 1 \mathrm{H}), 7.00(\mathrm{~d}, J=2.2 \mathrm{~Hz}, 1 \mathrm{H})$, $6.86-6.80(\mathrm{~m}, 2 \mathrm{H}), 6.40(\mathrm{~d}, J=15.8 \mathrm{~Hz}, 1 \mathrm{H}), 6.15(\mathrm{dd}, J=15.8,8.7 \mathrm{~Hz}, 1 \mathrm{H}), 3.80(\mathrm{~s}, 3 \mathrm{H})$, $3.64(\mathrm{~s}, 3 \mathrm{H}), 3.59-3.56(\mathrm{~m}, 1 \mathrm{H}), 3.33(\mathrm{dd}, J=14.6,7.8 \mathrm{~Hz}, 1 \mathrm{H}), 3.08(\mathrm{dd}, J=14.6,6.6 \mathrm{~Hz}$, $1 \mathrm{H})$.

${ }^{13} \mathbf{C ~ N M R}\left(151 \mathrm{MHz}, \mathrm{CDCl}_{3}\right) \delta 174.7,159.3,136.3,131.9,129.8,127.7,127.6,125.4,122.5$, $122.1,119.5,118.9,114.1,113.1,111.2,55.4,52.0,50.5,28.8$.

$\underline{\text { HRMS }}(\mathrm{m} / \mathrm{z})$ : $[\mathrm{M}+\mathrm{H}]^{+}$calcd for $\mathrm{C}_{21} \mathrm{H}_{22} \mathrm{NO}_{3}{ }^{+}, 336.1594$; found, 336.1592. 


\subsubsection{Synthesis and characterization of $3 p$}

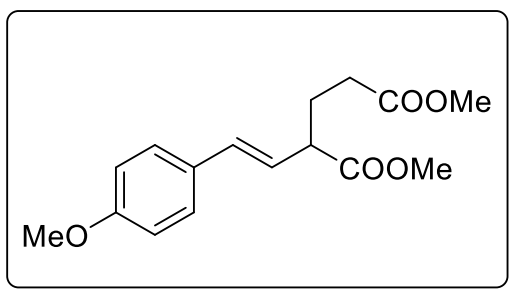

Compound 3p was synthesized according to the General procedure using pyridinium salt $\mathbf{6 k}(0.400 \mathrm{mmol}, 221 \mathrm{mg})$, nitrostyrene $\mathbf{1 b}(0.600 \mathrm{mmol}, 108 \mathrm{mg})$ in 1,4-dioxane $(2 \mathrm{ml}$, $0.2 \mathrm{M})$ and irradiated with blue light for $16 \mathrm{~h}$. The crude mixture was purified by silica gel chromatography (gradient: hexanes- $\mathrm{CH}_{2} \mathrm{Cl}_{2}$ ) to give $\mathbf{3 p}(48.6 \mathrm{mg}, 42 \%$ ) as a yellow oil. ${ }^{1} \mathbf{H}$ NMR $\left(600 \mathrm{MHz}, \mathrm{CDCl}_{3}\right) \delta 7.32-7.27(\mathrm{~m}, 2 \mathrm{H}), 6.87-$ $6.81(\mathrm{~m}, 2 \mathrm{H}), 6.43(\mathrm{~d}, J=15.8 \mathrm{~Hz}, 1 \mathrm{H}), 6.00(\mathrm{dd}, J=15.8,9.0 \mathrm{~Hz}, 1 \mathrm{H}), 3.80(\mathrm{~s}, 3 \mathrm{H}), 3.70$ (s, $3 \mathrm{H}), 3.65(\mathrm{~s}, 3 \mathrm{H}), 3.24-3.16(\mathrm{~m}, 1 \mathrm{H}), 2.41-2.32(\mathrm{~m}, 2 \mathrm{H}), 2.19-2.12(\mathrm{~m}, 1 \mathrm{H}), 2.02-1.94$ $(\mathrm{m}, 1 \mathrm{H})$.

${ }^{13} \mathbf{C ~ N M R}\left(151 \mathrm{MHz}, \mathrm{CDCl}_{3}\right) \delta 174.1,173.5,159.5,132.7,129.4,127.7,124.2,114.1,55.4$, 52.1, 51.8, 48.8, 31.6, 27.5.

$\underline{\text { HRMS }}(\mathrm{m} / \mathrm{z}):[\mathrm{M}+\mathrm{H}]^{+}$calcd for $\mathrm{C}_{16} \mathrm{H}_{21} \mathrm{O}_{5}{ }^{+}, 293.1384$; found, 293.1383 .

\subsubsection{Synthesis and characterization of $3 q$}

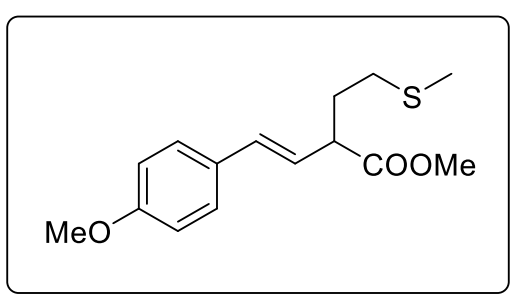

Compound $\mathbf{3 q}$ was synthesized according to the General procedure using pyridinium salt $6 \mathbf{1}(0.400 \mathrm{mmol}, 217 \mathrm{mg})$, nitrostyrene $\mathbf{1 b}(0.600 \mathrm{mmol}, 108 \mathrm{mg})$ in 1,4-dioxane $(2 \mathrm{ml}$, $0.2 \mathrm{M})$ and irradiated with blue light for $16 \mathrm{~h}$. The crude mixture was purified by silica gel chromatography (gradient: hexanes- $\mathrm{CH}_{2} \mathrm{Cl}_{2}$ ) to give $\mathbf{3 q}(42 \mathrm{mg}, 37 \%)$ as a yellow oil.

1H NMR $\left(600 \mathrm{MHz}, \mathrm{CDCl}_{3}\right) \delta 7.33-7.28(\mathrm{~m}, 2 \mathrm{H}), 6.88-$ $6.80(\mathrm{~m}, 2 \mathrm{H}), 6.45(\mathrm{~d}, J=15.8 \mathrm{~Hz}, 1 \mathrm{H}), 6.01(\mathrm{dd}, J=15.8,9.0 \mathrm{~Hz}, 1 \mathrm{H}), 3.80(\mathrm{~s}, 3 \mathrm{H}), 3.71(\mathrm{~s}$, $3 \mathrm{H}), 3.36-3.32(\mathrm{~m}, 1 \mathrm{H}), 2.58-2.46(\mathrm{~m}, 2 \mathrm{H}), 2.14(\mathrm{ddt}, J=13.7,8.3,6.8 \mathrm{~Hz}, 1 \mathrm{H}), 2.10(\mathrm{~s}$, $3 \mathrm{H}), 1.90(\mathrm{dtd}, J=13.8,8.1,5.8 \mathrm{~Hz}, 1 \mathrm{H})$.

${ }^{13} \mathbf{C ~ N M R}\left(151 \mathrm{MHz}, \mathrm{CDCl}_{3}\right) \delta 174.3,159.4,132.6,129.5,127.7,124.4,114.1,55.4,52.1$, 48.3, 31.7, 31.69, 15.5.

HRMS $(m / z):[\mathrm{M}+\mathrm{H}]^{+}$calcd for $\mathrm{C}_{15} \mathrm{H}_{21} \mathrm{O}_{3} \mathrm{~S}^{+}, 281.1206$; found, 281.1205.

\subsubsection{Synthesis and characterization of $3 r$}

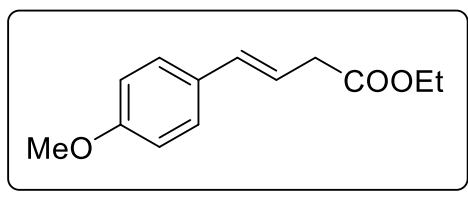

Compound $3 \mathbf{r}$ was synthesized according to the General procedure using pyridinium salt $\mathbf{6 m}(0.400 \mathrm{mmol}, 193 \mathrm{mg})$, nitrostyrene $\mathbf{1 b}(0.600 \mathrm{mmol}, 108 \mathrm{mg})$ in 1,4-dioxane ( $2 \mathrm{ml}, 0.2$ $\mathrm{M})$, and irradiated with blue light for $16 \mathrm{~h}$. The crude mixture was purified by silica gel chromatography (gradient: hexanes-

EtOAc) to give $3 \mathbf{r}(5 \mathrm{mg}, 6 \%)$ as a yellow oil.

${ }^{1} \mathbf{H ~ N M R}\left(600 \mathrm{MHz}, \mathrm{CDCl}_{3}\right) \delta 7.33-7.28(\mathrm{~m}, 2 \mathrm{H}), 6.87-6.81(\mathrm{~m}, 2 \mathrm{H}), 6.43(\mathrm{~d}, J=15.9 \mathrm{~Hz}$, $1 \mathrm{H}), 6.16(\mathrm{dt}, J=15.8,7.2 \mathrm{~Hz}, 1 \mathrm{H}), 4.17(\mathrm{q}, J=7.1 \mathrm{~Hz}, 2 \mathrm{H}), 3.80(\mathrm{~s}, 3 \mathrm{H}), 3.22-3.21(\mathrm{~m}$, $2 \mathrm{H}), 1.28(\mathrm{t}, J=7.2 \mathrm{~Hz}, 3 \mathrm{H})$.

${ }_{38 \text { NMR }}^{\text {13 }}\left(151 \mathrm{MHz}, \mathrm{CDCl}_{3}\right) \delta 172.0,159.3,132.9,129.9,127.6,119.8,114.1,60.9,55.4$, 38.7, 14.4 .

$\underline{\text { HRMS }}(\mathrm{m} / \mathrm{z}):[\mathrm{M}+\mathrm{H}]^{+}$calcd for $\mathrm{C}_{13} \mathrm{H}_{17} \mathrm{O}_{3}{ }^{+}, 221.1172$; found, 221.1172 .

All data are in good agreement with previously published data. ${ }^{21}$ 


\subsubsection{Synthesis and characterization of $3 \mathrm{~s}$}

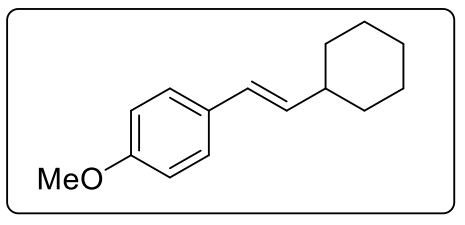

Compound 3s was synthesized according to the modified General procedure using pyridinium salt 6n $(0.20 \mathrm{mmol}, 96$ $\mathrm{mg})$, nitrostyrene $\mathbf{1 b}(0.30 \mathrm{mmol}, 54 \mathrm{mg})$ in EtOAc $(2 \mathrm{ml}, 0.1$ $\mathrm{M})$, and irradiated with blue light for $24 \mathrm{~h}$ at elevated temperature (range between $42-46^{\circ} \mathrm{C}$ ) in photoreactor (without fan). The crude mixture was purified by silica gel chromatography (hexanes-EtOAc 99:1) to give $3 \mathbf{s}(10 \mathrm{mg}, 23 \%)$ as a white solid.

${ }^{1}$ H NMR $\left(600 \mathrm{MHz}, \mathrm{CDCl}_{3}\right) \delta 7.30-7.25(\mathrm{~m}, 2 \mathrm{H}), 6.85-6.80(\mathrm{~m}, 2 \mathrm{H}), 6.28(\mathrm{~d}, J=15.8 \mathrm{~Hz}$, $1 \mathrm{H}), 6.03$ (dd, $J=15.8,7.0 \mathrm{~Hz}, 1 \mathrm{H}), 3.79$ (s, 3H), 2.10 (dddt, $J=17.3,10.6,7.0,3.5 \mathrm{~Hz}, 1 \mathrm{H}$ ), $1.82-1.73(\mathrm{~m}, 3 \mathrm{H}), 1.69-1.65(\mathrm{~m}, 1 \mathrm{H}), 1.36-1.11(\mathrm{~m}, 6 \mathrm{H})$.

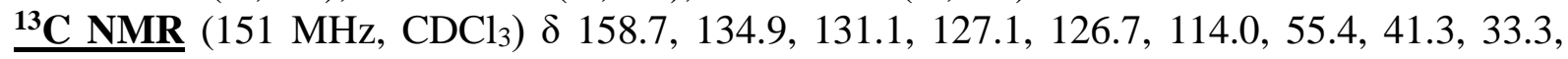
26.4, 26.2.

HRMS $(\mathrm{m} / \mathrm{z}):[\mathrm{M}+\mathrm{H}]^{+}$calcd for $\mathrm{C}_{15} \mathrm{H}_{21} \mathrm{O}^{+}, 217.1587$; found, 217.1586.

All data are in good agreement with previously published data. ${ }^{22}$

\subsubsection{Synthesis and characterization of $3 \mathrm{t}$}

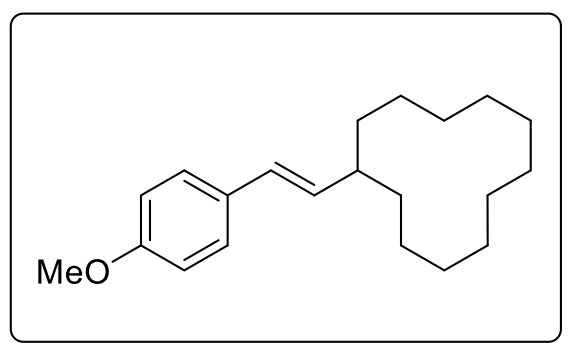

Compound 3t was synthesized according to the General procedure using pyridinium salt $\mathbf{6 0}(0.200 \mathrm{mmol}, 112$ $\mathrm{mg})$, nitrostyrene $\mathbf{1 b}(0.30 \mathrm{mmol}, 54 \mathrm{mg})$ in 1,4-dioxane $(2 \mathrm{ml}, 0.1 \mathrm{M})$, and irradiated with blue light for $16 \mathrm{~h}$. The crude mixture was purified by silica gel chromatography (hexanes-EtOAc 99:1) to give $\mathbf{3 t}(24.3 \mathrm{mg}, 40 \%)$ as a white solid.

${ }^{1} \mathbf{H}$ NMR $\left(600 \mathrm{MHz}, \mathrm{CDCl}_{3}\right) \delta 7.28(\mathrm{~d}, J=8.7 \mathrm{~Hz}, 2 \mathrm{H})$, $6.84(\mathrm{~d}, J=8.7 \mathrm{~Hz}, 2 \mathrm{H}), 6.28(\mathrm{~d}, J=15.8 \mathrm{~Hz}, 1 \mathrm{H}), 5.96(\mathrm{dd}, J=15.8,8.1 \mathrm{~Hz}, 1 \mathrm{H}), 3.80(\mathrm{~s}$, $3 \mathrm{H}), 2.34-2.28(\mathrm{~m}, 1 \mathrm{H}), 1.59-1.53(\mathrm{~m}, 2 \mathrm{H}), 1.61-1.23(\mathrm{~m}, 20 \mathrm{H})$.

${ }^{13} \mathbf{C}$ NMR $\left(151 \mathrm{MHz}, \mathrm{CDCl}_{3}\right) \delta 158.7,134.6,131.0,127.7,127.1,114.0,55.4,37.7,30.3$, 24.0, 24.0, 23.9, 23.6, 22.5

HRMS $(\mathrm{m} / z):[\mathrm{M}+\mathrm{H}]^{+}$calcd for $\mathrm{C}_{21} \mathrm{H}_{33} \mathrm{O}^{+}, 301.2526$; found, 301.2525.

\subsubsection{Synthesis and characterization of $3 u$}

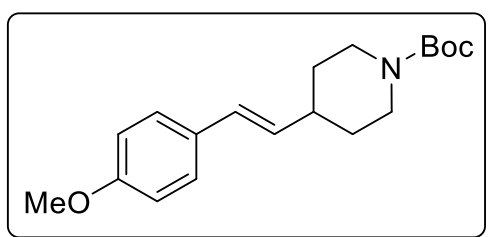

Compound $\mathbf{3 u}$ was synthesized according to the General procedure using pyridinium salt $\mathbf{6 p}(0.200 \mathrm{mmol}, 116 \mathrm{mg})$, nitrostyrene $\mathbf{1 b}(0.30 \mathrm{mmol}, 54 \mathrm{mg})$ in 1,4-dioxane $(2 \mathrm{ml}, 0.1$ $\mathrm{M})$, and irradiated with blue light for $24 \mathrm{~h}$. The crude mixture was purified by silica gel chromatography (hexanes-EtOAc 9:1) to give $3 \mathbf{u}(26.5 \mathrm{mg}, 42 \%)$ as a white solid.

${ }^{1} \mathbf{H}$ NMR $\left(600 \mathrm{MHz}, \mathrm{CDCl}_{3}\right) \delta 7.30-7.25(\mathrm{~m}, 2 \mathrm{H}), 6.86-6.81(\mathrm{~m}, 2 \mathrm{H}), 6.32(\mathrm{~d}, J=15.9 \mathrm{~Hz}$, $1 \mathrm{H}), 6.00(\mathrm{dd}, J=16.0,6.9 \mathrm{~Hz}, 1 \mathrm{H}), 4.20-4.04(\mathrm{~m}, 2 \mathrm{H}), 3.80(\mathrm{~s}, 3 \mathrm{H}), 2.81-2.77(\mathrm{~m}, 2 \mathrm{H})$, $2.29-2.20(\mathrm{~m}, 1 \mathrm{H}), 1.74(\mathrm{~d}, J=12.4 \mathrm{~Hz}, 2 \mathrm{H}), 1.47(\mathrm{~s}, 9 \mathrm{H}), 1.44-1.24(\mathrm{~m}, 2 \mathrm{H})$.

${ }^{13} \mathbf{C ~ N M R}\left(151 \mathrm{MHz}, \mathrm{CDCl}_{3}\right) \delta 159.0,155.0,132.4,130.4,127.9,127.2,114.1,79.4,55.4$, 43.6, 39.5, 32.1, 28.6.

$\underline{\text { HRMS }}(\mathrm{m} / \mathrm{z})$ : $[\mathrm{M}+\mathrm{Na}]^{+}$calcd for $\mathrm{C}_{19} \mathrm{H}_{27} \mathrm{NNaO}_{3}{ }^{+}, 340.1883$; found, 340.1878.

All data are in good agreement with previously published data. ${ }^{23}$ 


\subsubsection{Synthesis and characterization of $3 v$}

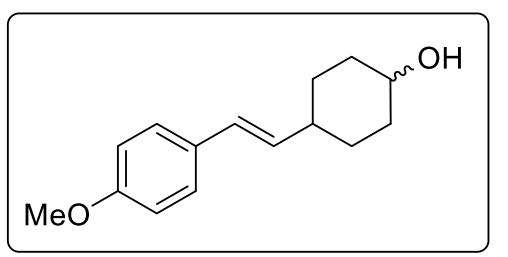

Compound $3 \mathbf{v}$ was synthesized according to the modified General procedure using pyridinium salt $\mathbf{6 q}(0.20 \mathrm{mmol}, 99$ $\mathrm{mg}$ ), nitrostyrene $\mathbf{1 b}$ ( 2 equiv., $0.40 \mathrm{mmol}, 72 \mathrm{mg}$ ) in 1,4dioxane $(2 \mathrm{ml}, 0.1 \mathrm{M})$, following by the addition of DIPEA (2 equiv., $0.40 \mathrm{mmol}, 53 \mathrm{mg}, 71 \mu \mathrm{l})$ and irradiated with blue light for $40 \mathrm{~h}$ at elevated temperature (range between $42-46{ }^{\circ} \mathrm{C}$ ) in a photoreactor (without fan). The crude mixture was purified by silica gel chromatography (hexanes-EtOAc 4:1) and then $\left(\mathrm{CH}_{2} \mathrm{Cl}_{2}\right.$-EtOAc 95:5) to give $\mathbf{3 v}$ as a mixture of diastereoisomers ( $\mathrm{dr} 1: 1)(7.5 \mathrm{mg}, 16 \%)$ as a yellow solid.

$\underline{{ }^{1} \mathbf{H ~ N M R}}\left(600 \mathrm{MHz}, \mathrm{CDCl}_{3}\right.$, both diastereoisomers reported) $\delta 7.30-7.24(\mathrm{~m}, 4 \mathrm{H}), 6.87-$ $6.79(\mathrm{~m}, 4 \mathrm{H}), 6.34-6.30(\mathrm{~m}, 2 \mathrm{H}), 6.08(\mathrm{dd}, J=16.0,6.9 \mathrm{~Hz}, 1 \mathrm{H}), 5.99(\mathrm{dd}, J=15.9,7.0 \mathrm{~Hz}$, $1 \mathrm{H}), 3.99-3.98(\mathrm{~m}, 1 \mathrm{H}), 3.80(\mathrm{~s}, 3 \mathrm{H}), 3.80(\mathrm{~s}, 3 \mathrm{H}), 3.63-3.57(\mathrm{~m}, 1 \mathrm{H}), 2.22-2.20(\mathrm{~m}, 1 \mathrm{H})$, $2.12-1.99(\mathrm{~m}, 3 \mathrm{H}), 1.90-1.83(\mathrm{~m}, 2 \mathrm{H}), 1.78-1.74(\mathrm{~m}, 2 \mathrm{H}), 1.66-1.59(\mathrm{~m}, 8 \mathrm{H}), 1.37-$ $1.22(\mathrm{~m}, 4 \mathrm{H})$.

${ }^{13} \mathbf{C ~ N M R}\left(151 \mathrm{MHz}, \mathrm{CDCl}_{3}\right.$, both diastereoisomers reported) $\delta 158.9,158.8,133.5,133.4$, $130.8,130.7,127.6,127.5,127.2,114.1,70.8,55.4,40.3,35.5,32.3,31.3,27.3$.

HRMS $(m / z):[\mathrm{M}+\mathrm{H}]^{+}$calcd for $\mathrm{C}_{15} \mathrm{H}_{21} \mathrm{O}_{2}{ }^{+}, 233.1536$; found, 233.1536.

\subsubsection{Synthesis and characterization of $3 w$}

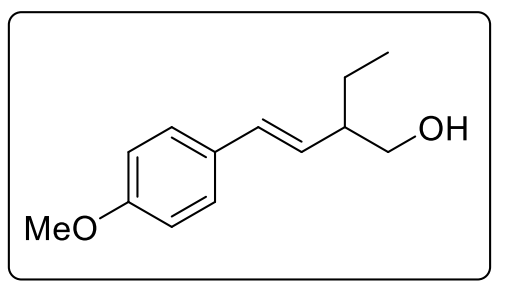

Compound 3w was synthesized according to the General procedure using pyridinium salt $6 \mathbf{r}(0.400 \mathrm{mmol}, 187 \mathrm{mg})$, nitrostyrene $\mathbf{1 b}(0.600 \mathrm{mmol}, 108 \mathrm{mg})$ in 1,4 -dioxane $(2 \mathrm{ml}$, $0.2 \mathrm{M}$ ) and irradiated with blue light for $18 \mathrm{~h}$ at elevated temperature (range between $42-46^{\circ} \mathrm{C}$ ) in photoreactor (without fan). The crude mixture was purified by silica gel chromatography (hexanes-EtOAc $4: 1)$ and then $\left(\mathrm{CH}_{2} \mathrm{Cl}_{2}-\right.$

EtOAc 95:5) to give $\mathbf{3 w}(18.3 \mathrm{mg}, 22 \%)$ as a pale yellow solid

$\underline{{ }^{1} \mathbf{H} ~ N M R}\left(600 \mathrm{MHz}, \mathrm{CDCl}_{3}\right) \delta 7.33-7.29(\mathrm{~m}, 2 \mathrm{H}), 6.87-6.83(\mathrm{~m}, 2 \mathrm{H}), 6.44(\mathrm{~d}, J=15.9 \mathrm{~Hz}$, $1 \mathrm{H}), 5.83(\mathrm{dd}, J=15.9,8.9 \mathrm{~Hz}, 1 \mathrm{H}), 3.81(\mathrm{~s}, 3 \mathrm{H}), 3.64(\mathrm{dd}, J=10.6,5.1 \mathrm{~Hz}, 1 \mathrm{H}), 3.50(\mathrm{dd}, J$ $=10.6,8.2 \mathrm{~Hz}, 1 \mathrm{H}), 2.31-2.23(\mathrm{~m}, 1 \mathrm{H}), 1.57-1.51(\mathrm{~m}, 1 \mathrm{H}), 1.40-1.31(\mathrm{~m}, 1 \mathrm{H}), 0.94(\mathrm{t}, J$ $=7.4 \mathrm{~Hz}, 3 \mathrm{H})$.

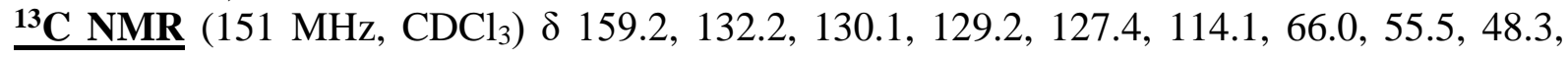
$24.3,11.9$.

HRMS $(m / z):[\mathrm{M}+\mathrm{H}]^{+}$calcd for $\mathrm{C}_{13} \mathrm{H}_{19} \mathrm{O}_{2}{ }^{+}, 207.1380$; found, 207.1380.

\subsubsection{Synthesis of $3 x$}

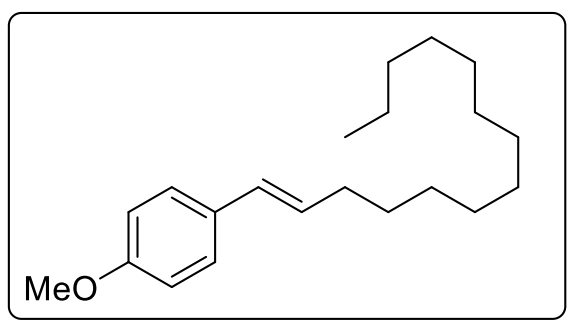

Compound 3x was attempted to be synthesized according to the General procedure using pyridinium salt $\mathbf{6 s}(0.400$ $\mathrm{mmol}, 225 \mathrm{mg})$, nitrostyrene $\mathbf{1 b}(0.600 \mathrm{mmol}, 108 \mathrm{mg})$ in 1,4-dioxane ( $2 \mathrm{ml}, 0.2 \mathrm{M})$, and irradiated with blue light for $24 \mathrm{~h}$ at elevated temperature (range between $42-46^{\circ} \mathrm{C}$ ) in photoreactor (without fan). No product $\mathbf{3 x}$ was formed according to a ${ }^{1} \mathrm{H}$ NMR analysis of the crude mixture. 


\subsubsection{Synthesis and characterization of $3 y$}

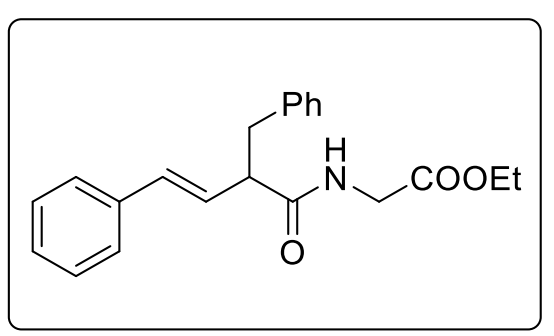

Compound 3y was synthesized according to the General procedure using pyridinium salt $6 \mathbf{t}(0.400 \mathrm{mmol}, 251 \mathrm{mg})$, nitrostyrene $1 \mathrm{a}(0.60 \mathrm{mmol}, 90 \mathrm{mg})$ in 1,4-dioxane $(2 \mathrm{ml}$, $0.2 \mathrm{M}$ ), and irradiated with blue light for $12 \mathrm{~h}$. The crude mixture was purified by silica gel chromatography (gradient $\mathrm{CH}_{2} \mathrm{Cl}_{2}$ to $\mathrm{CH}_{2} \mathrm{Cl}_{2}$-acetone 99:1) and then triturated with diethyl ether to give $\mathbf{3 y}(50 \mathrm{mg}, 37 \%)$ as

a white solid.

${ }_{1}^{1} \mathbf{H}$ NMR $\left(600 \mathrm{MHz}, \mathrm{CDCl}_{3}\right) \delta 7.34-7.16(\mathrm{~m}, 10 \mathrm{H}), 6.43(\mathrm{~d}, J=15.9 \mathrm{~Hz}, 1 \mathrm{H}), 6.25(\mathrm{dd}, J=$ $15.9,8.6 \mathrm{~Hz}, 1 \mathrm{H}), 6.02$ ("bs", $1 \mathrm{H}), 4.18$ (q, $J=7.1 \mathrm{~Hz}, 2 \mathrm{H}), 4.00$ (dd, $J=18.4,5.1 \mathrm{~Hz}, 1 \mathrm{H})$, $3.95(\mathrm{dd}, J=18.4,5.0 \mathrm{~Hz}, 1 \mathrm{H}), 3.31-3.26(\mathrm{~m}, 2 \mathrm{H}), 2.95-2.91(\mathrm{~m}, 1 \mathrm{H}), 1.25(\mathrm{t}, J=7.1 \mathrm{~Hz}$, $3 \mathrm{H})$.

$\underline{{ }^{13} \mathbf{C ~ N M R}}\left(151 \mathrm{MHz}, \mathrm{CDCl}_{3}\right) \delta 172.8,169.9,139.1,136.7,133.5,129.3,128.7,128.5,127.9$, 127.6, 126.5, 126.5, 61.7, 53.0, 41.6, 38.7, 14.2 .

HRMS $(m / z):[\mathrm{M}+\mathrm{H}]^{+}$calcd for $\mathrm{C}_{21} \mathrm{H}_{24} \mathrm{NO}_{3}{ }^{+}, 338.1751$; found, 338.1749.

\subsubsection{Synthesis and characterization of $3 z$}

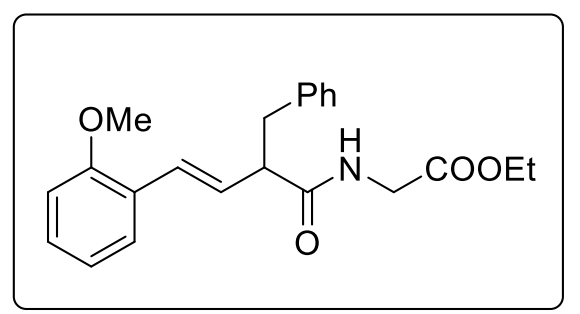

Compound $\mathbf{3 z}$ was synthesized according to the General procedure using pyridinium salt $6 \mathbf{t}(0.200 \mathrm{mmol}, 126 \mathrm{mg})$, nitrostyrene $1 \mathbf{d}(0.30 \mathrm{mmol}, 54 \mathrm{mg})$ in 1,4 -dioxane $(1 \mathrm{ml}$, $0.2 \mathrm{M}$ ), and irradiated with blue light for $12 \mathrm{~h}$. The crude mixture was purified by silica gel chromatography (hexanes-EtOAc $4: 1)$ to give $\mathbf{3 z}(38 \mathrm{mg}, 52 \%$ ) as a yellow oil.

${ }^{1} \mathbf{H}$ NMR $\left(600 \mathrm{MHz}, \mathrm{CDCl}_{3}\right) \delta 7.38(\mathrm{dd}, J=7.6,1.5 \mathrm{~Hz}, 1 \mathrm{H}), 7.27-7.23(\mathrm{~m}, 2 \mathrm{H}), 7.23-$ $7.15(\mathrm{~m}, 4 \mathrm{H}), 6.90(\mathrm{t}, J=7.5 \mathrm{~Hz}, 1 \mathrm{H}), 6.84(\mathrm{~d}, J=8.2 \mathrm{~Hz}, 1 \mathrm{H}), 6.76(\mathrm{~d}, J=16.0 \mathrm{~Hz}, 1 \mathrm{H})$, $6.26(\mathrm{dd}, J=16.0,8.7 \mathrm{~Hz}, 1 \mathrm{H}), 6.07(\mathrm{t}, J=4.3 \mathrm{~Hz}, 1 \mathrm{H}), 4.17(\mathrm{q}, J=7.1 \mathrm{~Hz}, 2 \mathrm{H}), 3.99(\mathrm{dd}, J$ $=18.4,5.1 \mathrm{~Hz}, 1 \mathrm{H}), 3.94(\mathrm{dd}, J=18.4,5.1 \mathrm{~Hz}, 1 \mathrm{H}), 3.81(\mathrm{~s}, 3 \mathrm{H}), 3.34-3.26(\mathrm{~m}, 2 \mathrm{H}), 2.94$ $(\mathrm{dd}, J=13.1,6.7 \mathrm{~Hz}, 1 \mathrm{H}), 1.24(\mathrm{t}, J=7.2 \mathrm{~Hz}, 3 \mathrm{H})$

${ }^{13} \mathbf{C ~ N M R}\left(151 \mathrm{MHz}, \mathrm{CDCl}_{3}\right) \delta 173.1,169.9,156.7,139.3,129.4,128.9,128.4,128.4,128.2$, 127.0, 126.4, 125.7, 120.8, 111.0, 61.6, 55.6, 53.5, 41.6, 38.7, 14.2.

$\underline{\text { HRMS }}(\mathrm{m} / \mathrm{z}):[\mathrm{M}+\mathrm{H}]^{+}$calcd for $\mathrm{C}_{22} \mathrm{H}_{26} \mathrm{NO}_{4}{ }^{+}, 368.1856$; found, 368.1853.

\subsubsection{Synthesis and characterization of 3aa}

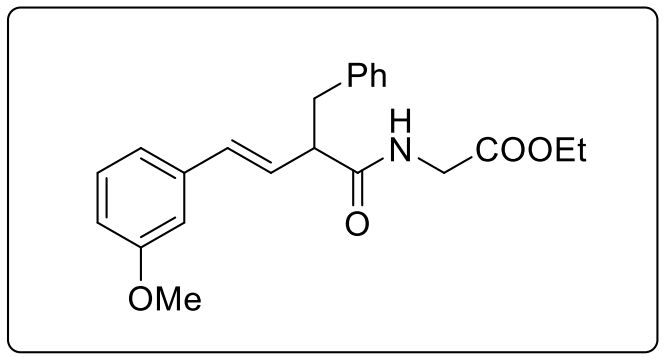

Compound 3aa was synthesized according to the General procedure using pyridinium salt $6 \mathbf{t}(0.200$ $\mathrm{mmol}, 126 \mathrm{mg})$, nitrostyrene $1 \mathrm{c}(0.30 \mathrm{mmol}, 54 \mathrm{mg})$ in 1,4-dioxane $(1 \mathrm{ml}, 0.2 \mathrm{M})$, and irradiated with blue light for $12 \mathrm{~h}$. The crude mixture was purified by silica gel chromatography (hexanes-EtOAc $4: 1$ ) to give 3aa (40 $\mathrm{mg}, 54 \%)$ as a yellow oil.

$\underline{{ }^{1} \mathbf{H} ~ N M R}\left(600 \mathrm{MHz}, \mathrm{CDCl}_{3}\right) \delta 7.27-7.23(\mathrm{~m}, 2 \mathrm{H}), 7.22-7.16(\mathrm{~m}, 4 \mathrm{H}), 6.91(\mathrm{~d}, J=7.6 \mathrm{~Hz}$, $1 \mathrm{H}), 6.87-6.84(\mathrm{~m}, 1 \mathrm{H}), 6.80-6.77(\mathrm{~m}, 1 \mathrm{H}), 6.40(\mathrm{~d}, J=15.9 \mathrm{~Hz}, 1 \mathrm{H}), 6.25(\mathrm{dd}, J=15.8$, $8.5 \mathrm{~Hz}, 1 \mathrm{H}), 6.03(\mathrm{t}, J=4.5 \mathrm{~Hz}, 1 \mathrm{H}), 4.18(\mathrm{q}, J=7.2 \mathrm{~Hz}, 2 \mathrm{H}), 4.00(\mathrm{dd}, J=18.4,5.1 \mathrm{~Hz}, 1 \mathrm{H})$, 
$3.95(\mathrm{dd}, J=18.4,5.0 \mathrm{~Hz}, 1 \mathrm{H}), 3.80(\mathrm{~s}, 3 \mathrm{H}), 3.31-3.24(\mathrm{~m}, 2 \mathrm{H}), 2.95-2.89(\mathrm{~m}, 1 \mathrm{H}), 1.25(\mathrm{t}$, $J=7.1 \mathrm{~Hz}, 3 \mathrm{H})$.

${ }^{13} \mathbf{C ~ N M R}\left(151 \mathrm{MHz}, \mathrm{CDCl}_{3}\right) \delta 172.9,169.9,159.9,139.1,138.1,133.3,129.7,129.3,128.5$, $127.9,126.5,119.2,113.6,111.7,61.7,55.4,53.1,41.6,38.7,14.2$.

HRMS $(\mathrm{m} / \mathrm{z}):[\mathrm{M}+\mathrm{H}]^{+}$calcd for $\mathrm{C}_{22} \mathrm{H}_{26} \mathrm{NO}_{4}{ }^{+}, 368.1856$; found, 368.1853.

\subsubsection{Synthesis and characterization of 3ab}

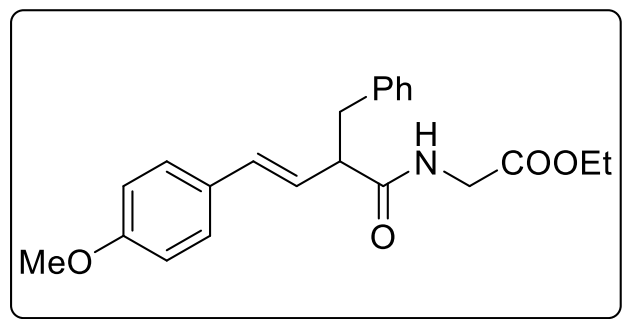

(43 $\mathrm{mg}, 58 \%)$ as a pale yellow solid.

$\underline{{ }_{1} \mathbf{H ~ N M R}}\left(600 \mathrm{MHz}, \mathrm{CDCl}_{3}\right) \delta 7.28-7.23(\mathrm{~m}, 4 \mathrm{H}), 7.21-7.17(\mathrm{~m}, 3 \mathrm{H}), 6.85-6.81(\mathrm{~m}, 2 \mathrm{H})$, $6.37(\mathrm{~d}, J=15.8 \mathrm{~Hz}, 1 \mathrm{H}), 6.10(\mathrm{dd}, J=15.8,8.4 \mathrm{~Hz}, 1 \mathrm{H}), 6.02(\mathrm{t}, J=4.7 \mathrm{~Hz}, 1 \mathrm{H}), 4.18(\mathrm{q}, J$ $=7.2 \mathrm{~Hz}, 2 \mathrm{H}), 4.00(\mathrm{dd}, J=18.4,5.1 \mathrm{~Hz}, 1 \mathrm{H}), 3.95(\mathrm{dd}, J=18.4,5.0 \mathrm{~Hz}, 1 \mathrm{H}), 3.80(\mathrm{~s}, 3 \mathrm{H})$, $3.29-3.24(\mathrm{~m}, 2 \mathrm{H}), 2.94-2.87(\mathrm{~m}, 1 \mathrm{H}), 1.25(\mathrm{t}, J=7.1 \mathrm{~Hz}, 3 \mathrm{H})$.

${ }_{13}^{13}$ NMR $\left(151 \mathrm{MHz}, \mathrm{CDCl}_{3}\right) \delta 173.2,169.9,159.4,139.3,132.9,129.5,129.3,128.5,127.7$, 126.4, 125.3, 114.1, 61.6, 55.4, 53.0, 41.6, 38.7, 14.2 .

HRMS $(m / z):[\mathrm{M}+\mathrm{H}]^{+}$calcd for $\mathrm{C}_{22} \mathrm{H}_{26} \mathrm{NO}_{4}{ }^{+}, 368.1856$; found, 368.1853.

\subsubsection{Synthesis and characterization of 3ac}

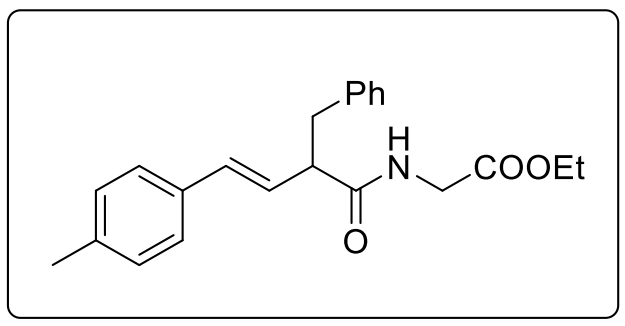

yellow solid.
Compound 3ac was synthesized according to the General procedure using pyridinium salt 6t $(0.400$ mmol, $251 \mathrm{mg})$, nitrostyrene $1 \mathrm{e}(0.60 \mathrm{mmol}, 98 \mathrm{mg})$ in 1,4-dioxane $(2 \mathrm{ml}, 0.2 \mathrm{M})$ and irradiated with blue light for $24 \mathrm{~h}$. The crude mixture was purified by silica gel chromatography (gradient $\mathrm{CH}_{2} \mathrm{Cl}_{2}$ to $\mathrm{CH}_{2} \mathrm{Cl}_{2}$ acetone 99:1) to give $\mathbf{3 a c}(64 \mathrm{mg}, 46 \%)$ as a pale

${ }^{1}$ H NMR $\left(600 \mathrm{MHz}, \mathrm{CDCl}_{3}\right) \delta 7.26-7.16(\mathrm{~m}, 7 \mathrm{H}), 7.10(\mathrm{~d}, J=7.9 \mathrm{~Hz}, 2 \mathrm{H}), 6.39(\mathrm{~d}, J=15.9$ $\mathrm{Hz}, 1 \mathrm{H}), 6.19(\mathrm{dd}, J=15.9,8.2 \mathrm{~Hz}, 1 \mathrm{H}), 6.04(\mathrm{t}, J=4.6 \mathrm{~Hz}, 1 \mathrm{H}), 4.17(\mathrm{q}, J=7.1 \mathrm{~Hz}, 2 \mathrm{H})$, $4.00(\mathrm{dd}, J=18.4,5.1 \mathrm{~Hz}, 1 \mathrm{H}), 3.95(\mathrm{dd}, J=18.4,5.1 \mathrm{~Hz}, 1 \mathrm{H}), 3.29-3.25(\mathrm{~m}, 2 \mathrm{H}), 2.94-$ $2.90(\mathrm{~m}, 1 \mathrm{H}), 2.32$ (s, 3H), 1.25 (t, $J=7.2 \mathrm{~Hz}, 3 \mathrm{H})$.

${ }_{13}^{13}$ NMR $\left(151 \mathrm{MHz}, \mathrm{CDCl}_{3}\right) \delta 173.0,169.9,139.2,137.7,133.9,133.4,129.4,129.3,128.5$, 126.5, 126.4, 126.4, 61.6, 53.0, 41.6, 38.7, 21.3, 14.2.

HRMS $(m / z):[\mathrm{M}+\mathrm{H}]^{+}$calcd for $\mathrm{C}_{22} \mathrm{H}_{26} \mathrm{NO}_{3}{ }^{+}, 352.1907$; found, 352.1904 .

\subsubsection{Synthesis and characterization of 3ad}

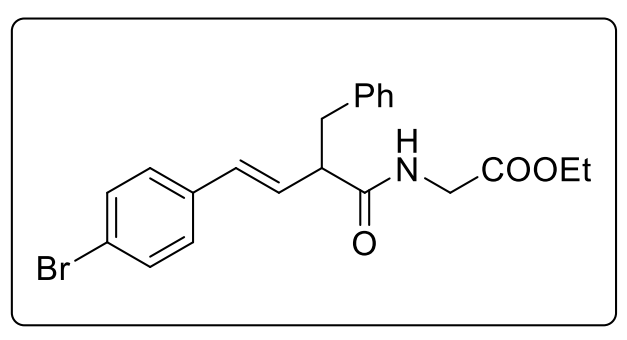

Compound 3ad was synthesized according to the General procedure using pyridinium salt $6 \mathbf{t}(0.400$ mmol, $251 \mathrm{mg})$, nitrostyrene $\mathbf{1 f}(0.600 \mathrm{mmol}, 137 \mathrm{mg})$ in 1,4-dioxane ( $2 \mathrm{ml}, 0.2 \mathrm{M})$, and irradiated with blue light for $12 \mathrm{~h}$. The crude mixture was purified by silica gel chromatography (gradient $\mathrm{CH}_{2} \mathrm{Cl}_{2}$ to $\mathrm{CH}_{2} \mathrm{Cl}_{2-}$ 
acetone 99:1) and then triturated with diethyl ether to give 3ad (68 mg, 41\%) as a white solid. $\underline{{ }^{1} \mathbf{H ~ N M R}}\left(600 \mathrm{MHz}, \mathrm{CDCl}_{3}\right) \delta 7.41(\mathrm{~d}, J=8.4 \mathrm{~Hz}, 2 \mathrm{H}), 7.26-7.16(\mathrm{~m}, 7 \mathrm{H}), 6.35(\mathrm{~d}, J=15.9$ $\mathrm{Hz}, 1 \mathrm{H}), 6.25$ (dd, $J=15.9,8.3 \mathrm{~Hz}, 1 \mathrm{H}), 5.99$ ("bs", $1 \mathrm{H}), 4.18$ (q, $J=7.1 \mathrm{~Hz}, 2 \mathrm{H}), 4.01$ (dd, $J$ $=18.4,5.2 \mathrm{~Hz}, 1 \mathrm{H}), 3.94(\mathrm{dd}, J=18.4,4.9 \mathrm{~Hz}, 1 \mathrm{H}), 3.29-3.24(\mathrm{~m}, 2 \mathrm{H}), 2.98-2.94(\mathrm{~m}, 1 \mathrm{H})$, $1.25(\mathrm{t}, J=7.1 \mathrm{~Hz}, 3 \mathrm{H})$.

$\underline{{ }^{13} \mathrm{C} \mathrm{NMR}}\left(151 \mathrm{MHz}, \mathrm{CDCl}_{3}\right) \delta 172.6,169.9,139.0,135.6,132.1,131.8,129.3,128.5,128.5$, 128.0, 126.57, 121.6, 61.7, 53.1, 41.6, 38.8, 14.3 .

HRMS $(\mathrm{m} / z):[\mathrm{M}+\mathrm{H}]^{+}$calcd for $\mathrm{C}_{21} \mathrm{H}_{23} \mathrm{BrNO}_{3}{ }^{+}, 416.0856$; found, 416.0854.

\subsubsection{Synthesis and characterization of 3ae}

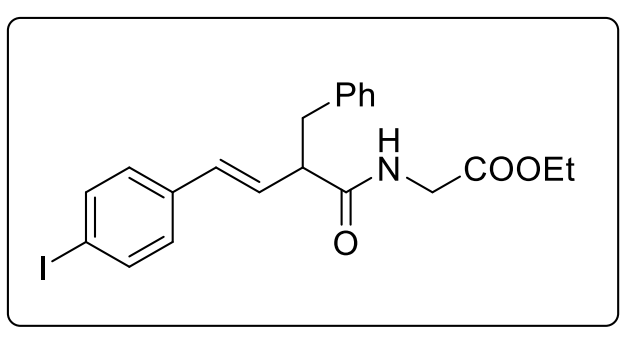

Compound 3ae was synthesized according to the General procedure using pyridinium salt 6t $(0.400$ mmol, $251 \mathrm{mg})$, nitrostyrene $1 \mathrm{~g}(0.600 \mathrm{mmol}, 165 \mathrm{mg})$ in 1,4-dioxane $(2 \mathrm{ml}, 0.2 \mathrm{M})$, and irradiated with blue light for $14 \mathrm{~h}$. The crude mixture was purified by silica gel chromatography (gradient $\mathrm{CH}_{2} \mathrm{Cl}_{2}$ to $\mathrm{CH}_{2} \mathrm{Cl}_{2}$ acetone 99:1) and then triturated with diethyl ether to give 3ae $(81 \mathrm{mg}, 44 \%)$ as a pale yellow solid.

$\underline{{ }^{1} \mathbf{H} \text { NMR }}\left(600 \mathrm{MHz}, \mathrm{CDCl}_{3}\right) \delta 7.61(\mathrm{~d}, J=8.3 \mathrm{~Hz}, 2 \mathrm{H}), 7.27-7.16(\mathrm{~m}, 5 \mathrm{H}), 7.05(\mathrm{~d}, J=8.3$ $\mathrm{Hz}, 2 \mathrm{H}), 6.33$ (d, $J=15.9 \mathrm{~Hz}, 1 \mathrm{H}), 6.26$ (dd, $J=15.9,8.0 \mathrm{~Hz}, 1 \mathrm{H}), 5.98$ ("bs", $1 \mathrm{H}), 4.18$ (q, $J$ $=7.1 \mathrm{~Hz}, 2 \mathrm{H}), 4.01(\mathrm{dd}, J=18.4,5.1 \mathrm{~Hz}, 1 \mathrm{H}), 3.94(\mathrm{dd}, J=18.4,4.9 \mathrm{~Hz}, 1 \mathrm{H}), 3.28-3.23(\mathrm{~m}$, $2 \mathrm{H}), 2.93-2.89(\mathrm{~m}, 1 \mathrm{H}), 1.25(\mathrm{t}, J=7.1 \mathrm{~Hz}, 3 \mathrm{H})$.

${ }^{13} \mathbf{C ~ N M R}\left(151 \mathrm{MHz}, \mathrm{CDCl}_{3}\right) \delta 172.6,169.9,138.9,137.8,136.2,132.2,129.3,128.6,128.5$, 128.3, 126.6, 93.1, 61.7, 53.1, 41.6, 38.8, 14.3.

$\underline{\text { HRMS }}(\mathrm{m} / \mathrm{z}):[\mathrm{M}+\mathrm{H}]^{+}$calcd for $\mathrm{C}_{21} \mathrm{H}_{23} \mathrm{INO}_{3}{ }^{+}, 464.0717$; found, 464.0715.

\subsubsection{Synthesis and characterization of 3af}

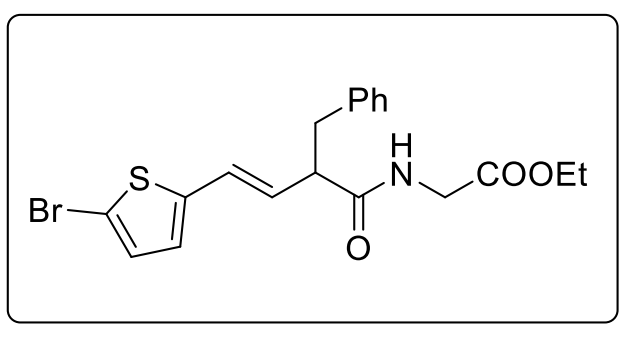

${ }^{1} \mathbf{H}$ NMR $\left(600 \mathrm{MHz}, \mathrm{CDCl}_{3}\right) \delta 7.28-7.24(\mathrm{~m}, 2 \mathrm{H}), 7.21-7.14(\mathrm{~m}, 3 \mathrm{H}), 6.87(\mathrm{~d}, J=3.8 \mathrm{~Hz}$, $1 \mathrm{H}), 6.62(\mathrm{~d}, J=3.8 \mathrm{~Hz}, 1 \mathrm{H}), 6.42(\mathrm{~d}, J=15.7 \mathrm{~Hz}, 1 \mathrm{H}), 6.02-5.95(\mathrm{~m}, 2 \mathrm{H}), 4.19(\mathrm{q}, J=7.2$ $\mathrm{Hz}, 2 \mathrm{H}), 4.00(\mathrm{dd}, J=18.4,5.1 \mathrm{~Hz}, 1 \mathrm{H}), 3.94(\mathrm{dd}, J=18.4,5.0 \mathrm{~Hz}, 1 \mathrm{H}), 3.26-3.17(\mathrm{~m}, 2 \mathrm{H})$, $2.92-2.85(\mathrm{~m}, 1 \mathrm{H}), 1.26(\mathrm{t}, J=7.1 \mathrm{~Hz}, 3 \mathrm{H})$.

$\underline{{ }^{13} \mathbf{C ~ N M R}}\left(151 \mathrm{MHz}, \mathrm{CDCl}_{3}\right) \delta 172.4,169.8,143.3,138.8,130.3,129.3,128.6,127.6,126.6$, 126.1, 125.9, 111.3, 61.7, 52.8, 41.6, 38.7, 14.3.

$\underline{\text { HRMS }}(\mathrm{m} / \mathrm{z}):[\mathrm{M}+\mathrm{H}]^{+}$calcd for $\mathrm{C}_{19} \mathrm{H}_{21} \mathrm{BrNO}_{3} \mathrm{~S}^{+}, 422.0420$; found, 422.0418 . 


\subsubsection{Synthesis and characterization of $3 \mathrm{ag}$}

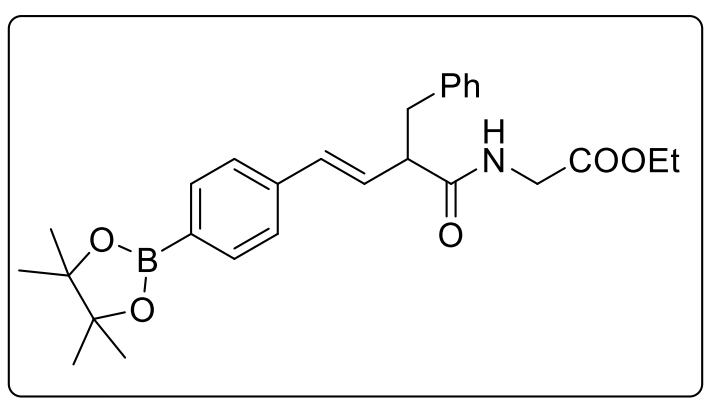

Compound 3ag was synthesized according to the General procedure using pyridinium salt 6t (0.200 mmol, $126 \mathrm{mg})$, nitrostyrene $\mathbf{1 i}(0.30$ $\mathrm{mmol}, 83 \mathrm{mg})$ in 1,4-dioxane $(1 \mathrm{ml}, 0.2 \mathrm{M})$, and irradiated with blue light for $12 \mathrm{~h}$. The crude mixture was purified by silica gel chromatography (hexanes-EtOAc 4:1 and then $\mathrm{CH}_{2} \mathrm{Cl}_{2}$-EtOAc 95:5) to give $\mathbf{3 a g}(23 \mathrm{mg}, 25 \%$ ) as a colorless oil.

$\underline{{ }^{1} \mathbf{H} \text { NMR }}\left(600 \mathrm{MHz}, \mathrm{CDCl}_{3}\right) \delta 7.73(\mathrm{~d}, J=8.1 \mathrm{~Hz}, 2 \mathrm{H}), 7.32(\mathrm{~d}, J=8.1 \mathrm{~Hz}, 2 \mathrm{H}), 7.28-7.22$ $(\mathrm{m}, 2 \mathrm{H}), 7.21-7.14(\mathrm{~m}, 3 \mathrm{H}), 6.42(\mathrm{~d}, J=15.9 \mathrm{~Hz}, 1 \mathrm{H}), 6.32(\mathrm{dd}, J=15.9,8.5 \mathrm{~Hz}, 1 \mathrm{H}), 6.01$ $(\mathrm{t}, J=4.9 \mathrm{~Hz}, 1 \mathrm{H}), 4.18(\mathrm{q}, J=7.1 \mathrm{~Hz}, 2 \mathrm{H}), 4.00(\mathrm{dd}, J=18.4,5.1 \mathrm{~Hz}, 1 \mathrm{H}), 3.95(\mathrm{dd}, J=$ $18.4,5.0 \mathrm{~Hz}, 1 \mathrm{H}), 3.32-3.24(\mathrm{~m}, 2 \mathrm{H}), 2.96-2.89(\mathrm{~m}, 1 \mathrm{H}), 1.34(\mathrm{~s}, 12 \mathrm{H}), 1.25(\mathrm{t}, J=7.2 \mathrm{~Hz}$, $3 \mathrm{H})$.

${ }^{13} \mathbf{C ~ N M R}\left(151 \mathrm{MHz}, \mathrm{CDCl}_{3}\right) \delta 172.8,169.8,139.4,139.1,135.2,133.4,129.3,128.7,128.5$, $126.5,125.8,83.9,61.7,53.2,41.6,38.8,25.0,25.0,14.3$.

HRMS $(\mathrm{m} / \mathrm{z}):[\mathrm{M}+\mathrm{H}]^{+}$calcd for $\mathrm{C}_{27} \mathrm{H}_{35} \mathrm{BNO}_{5}{ }^{+}, 464.2603$; found, 464.2601.

\subsubsection{Synthesis and characterization of 3ah}

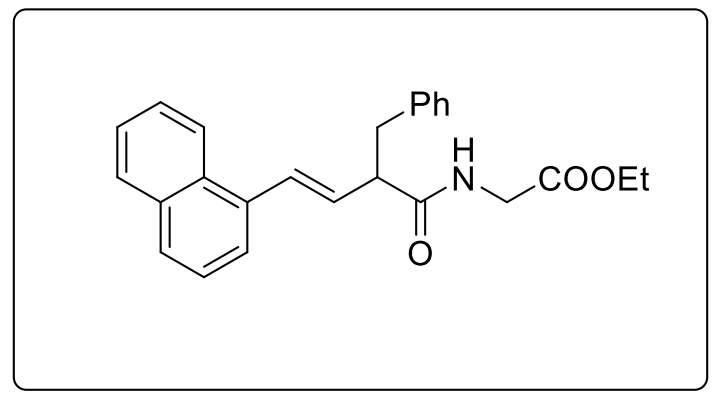

Compound 3ah was synthesized according to the General procedure using pyridinium salt $6 \mathbf{t}$ (0.400 mmol, $251 \mathrm{mg})$, nitrostyrene $\mathbf{1 j}(0.60$ $\mathrm{mmol}, 120 \mathrm{mg})$ in 1,4-dioxane $(2 \mathrm{ml}, 0.2 \mathrm{M})$, and irradiated with blue light for $20 \mathrm{~h}$. The crude mixture was purified by silica gel chromatography (gradient $\mathrm{CH}_{2} \mathrm{Cl}_{2}$ to $\mathrm{CH}_{2} \mathrm{Cl}_{2}-$ acetone 99:1 and then hexanes-EtOAc 4:1) to give 3ah (55 mg, 36\%) as a yellow oil.

${ }^{1} \mathbf{H}$ NMR $\left(300 \mathrm{MHz}, \mathrm{CDCl}_{3}\right) \delta 7.87-7.68(\mathrm{~m}, 3 \mathrm{H}), 7.56-7.36(\mathrm{~m}, 4 \mathrm{H}), 7.34-7.17(\mathrm{~m}, 5 \mathrm{H})$, $7.10(\mathrm{~d}, J=15.7 \mathrm{~Hz}, 1 \mathrm{H}), 6.24(\mathrm{dd}, J=15.6,8.9 \mathrm{~Hz}, 1 \mathrm{H}), 6.17(\mathrm{bt}, J=5.1 \mathrm{~Hz}, 1 \mathrm{H}), 4.21(\mathrm{q}, J$ $=7.1 \mathrm{~Hz}, 2 \mathrm{H}), 4.08(\mathrm{dd}, J=18.4,5.1 \mathrm{~Hz}, 1 \mathrm{H}), 4.01(\mathrm{dd}, J=18.4,5.1 \mathrm{~Hz}, 1 \mathrm{H}), 3.51-3.41$ $(\mathrm{m}, 1 \mathrm{H}), 3.37(\mathrm{dd}, J=13.5,6.4 \mathrm{~Hz}, 1 \mathrm{H}), 2.99(\mathrm{dd}, J=13.4,8.1 \mathrm{~Hz}, 1 \mathrm{H}), 1.27(\mathrm{t}, J=7.1 \mathrm{~Hz}$, $3 \mathrm{H})$.

${ }^{13} \mathbf{C ~ N M R}\left(75 \mathrm{MHz}, \mathrm{CDCl}_{3}\right) \delta 172.9,169.9,139.2,134.6,133.6,131.3,131.1,130.6,129.4$, 128.6, 128.5, 128.2, 126.5, 126.1, 125.9, 125.7, 124.2, 124.0, 61.7, 53.3, 41.7, 38.7, 14.2.

HRMS $(m / z):[\mathrm{M}+\mathrm{H}]^{+}$calcd for $\mathrm{C}_{25} \mathrm{H}_{26} \mathrm{NO}_{3}{ }^{+}, 388.1907$; found, 388.1906. 


\subsubsection{Synthesis and characterization of 3ai}

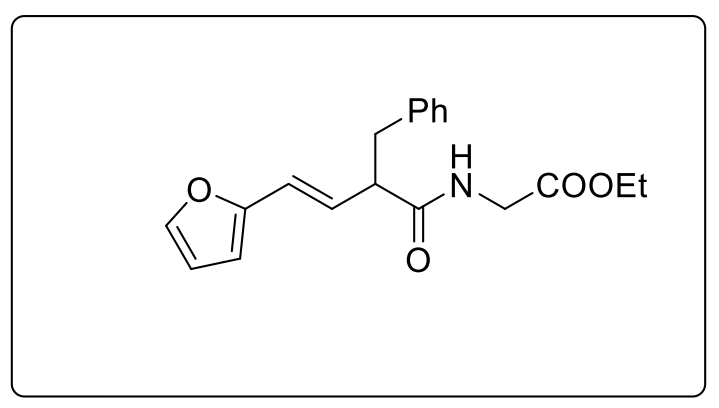

Compound 3ai was synthesized according to the General procedure using pyridinium salt 6t $(0.200 \mathrm{mmol}, 126 \mathrm{mg})$, nitrostyrene $\mathbf{1 k}(0.30$ mmol, $42 \mathrm{mg}$ ) and DIPEA (2 eq., $0.400 \mathrm{mmol}, 52$ $\mathrm{mg}, 70 \mu \mathrm{L})$ in 1,4-dioxane $(1 \mathrm{ml}, 0.2 \mathrm{M})$, and irradiated with blue light for $12 \mathrm{~h}$. The crude mixture was purified by silica gel chromatography (gradient $\mathrm{CH}_{2} \mathrm{Cl}_{2}$ to $\mathrm{CH}_{2} \mathrm{Cl}_{2}-$ acetone 99:1 and then hexanes-EtOAc 4:1) to

give 3ai (17 mg, 25\%) as a pale-yellow solid.

${ }^{1} \mathbf{H}$ NMR $\left(300 \mathrm{MHz}, \mathrm{CDCl}_{3}\right) \delta 7.32(\mathrm{~d}, J=2.0 \mathrm{~Hz}, 1 \mathrm{H}), 7.28-7.15(\mathrm{~m}, 5 \mathrm{H}), 6.34(\mathrm{dd}, J=$ $3.3,1.9 \mathrm{~Hz}, 1 \mathrm{H}), 6.31-6.17(\mathrm{~m}, 3 \mathrm{H}), 6.04(\mathrm{bt}, J=5.2 \mathrm{~Hz}, 1 \mathrm{H}), 4.18(\mathrm{q}, J=7.1 \mathrm{~Hz}, 2 \mathrm{H}), 4.07$ $-3.87(\mathrm{~m}, 2 \mathrm{H}), 3.33-3.18(\mathrm{~m}, 2 \mathrm{H}), 2.97-2.84(\mathrm{~m}, 1 \mathrm{H}), 1.25(\mathrm{t}, J=7.1 \mathrm{~Hz}, 3 \mathrm{H})$.

${ }^{13} \mathbf{C ~ N M R}\left(75 \mathrm{MHz}, \mathrm{CDCl}_{3}\right) \delta 172.7,169.9,152.2,142.2,139.1,129.3,128.5,126.5,126.0$, 121.8, 111.4, 108.2, 61.7, 52.7, 41.6, 38.6, 14.2.

$\underline{\text { HRMS }}(\mathrm{m} / \mathrm{z})$ : $[\mathrm{M}+\mathrm{H}]^{+}$calcd for $\mathrm{C}_{19} \mathrm{H}_{22} \mathrm{NO}_{4}{ }^{+}, 328.1543$; found, 328.1542.

\subsubsection{Synthesis and characterization of 3aj}

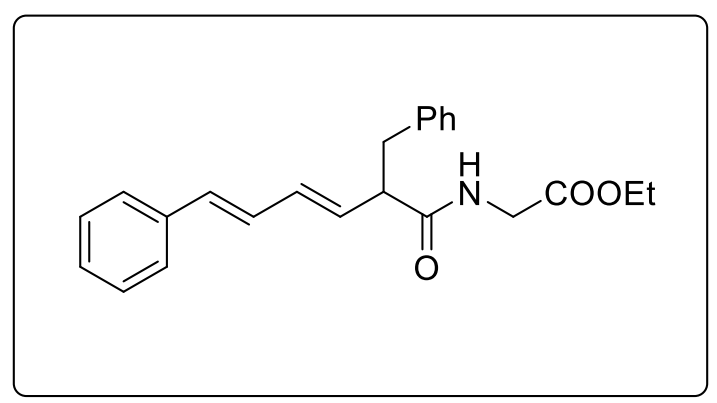

Compound 3aj was synthesized according to the General procedure using pyridinium salt $6 \mathbf{t}$ (0.400 mmol, $251 \mathrm{mg})$, nitrostyrene $1 \mathrm{ll}(0.60$ mmol, $105 \mathrm{mg})$ and DIPEA (0.800 mmol, 103 $\mathrm{mg}, 139 \mu \mathrm{L})$ in 1,4-dioxane $(2 \mathrm{ml}, 0.2 \mathrm{M})$, and irradiated with blue light for $18 \mathrm{~h}$. The crude mixture was purified by silica gel chromatography (gradient $\mathrm{CH}_{2} \mathrm{Cl}_{2}$ to $\mathrm{CH}_{2} \mathrm{Cl}_{2-}$ acetone 99:1 and then hexanes-EtOAc 4:1) to give 3aj (64 mg, 44\%) as a pale-yellow solid.

$\underline{{ }^{1} \mathbf{H} \text { NMR }}\left(300 \mathrm{MHz}, \mathrm{CDCl}_{3}\right) \delta 7.43-7.13(\mathrm{~m}, 10 \mathrm{H}), 6.72(\mathrm{ddd}, J=15.6,10.4,0.9 \mathrm{~Hz}, 1 \mathrm{H})$, $6.47(\mathrm{~d}, J=15.7 \mathrm{~Hz}, 1 \mathrm{H}), 6.25(\mathrm{ddt}, J=15.3,10.5,0.8 \mathrm{~Hz}, 1 \mathrm{H}), 6.02-5.97$ (bt, $J=4.6 \mathrm{~Hz}$, $1 \mathrm{H}), 5.84(\mathrm{dd}, J=15.3,8.5 \mathrm{~Hz}, 1 \mathrm{H}), 4.19(\mathrm{q}, J=7.2 \mathrm{~Hz}, 2 \mathrm{H}), 4.02(\mathrm{dd}, J=18.4,5.1 \mathrm{~Hz}, 1 \mathrm{H})$, $3.94(\mathrm{dd}, J=18.5,4.9 \mathrm{~Hz}, 1 \mathrm{H}), 3.27-3.20(\mathrm{~m}, 2 \mathrm{H}), 2.92-2.85(\mathrm{~m}, 1 \mathrm{H}), 1.26(\mathrm{t}, J=7.2 \mathrm{~Hz}$, $3 \mathrm{H})$.

${ }^{13} \mathbf{C ~ N M R}\left(75 \mathrm{MHz}, \mathrm{CDCl}_{3}\right) \delta 172.8,169.9,139.1,137.2,134.0,132.8,131.4,129.3,128.7$, $128.5,128.2,127.8,126.5,126.5,61.7,52.8,41.6,38.6,14.2$

HRMS $(m / z):[\mathrm{M}+\mathrm{H}]^{+}$calcd for $\mathrm{C}_{23} \mathrm{H}_{26} \mathrm{NO}_{3}{ }^{+}, 364.1907$; found, 364.1906. 


\subsubsection{Synthesis and characterization of 3an}

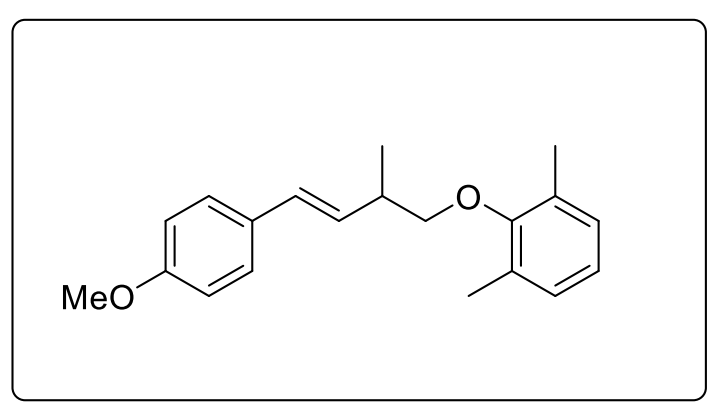

Compound 3an was synthesized according to the General procedure using pyridinium salt $\mathbf{6 w}$ (0.200 mmol, $112 \mathrm{mg})$, nitrostyrene $\mathbf{1 b}(0.30$ mmol, $54 \mathrm{mg})$ and DIPEA $(0.40 \mathrm{mmol}, 52 \mathrm{mg}$, $70 \mu \mathrm{L})$ in 1,4-dioxane $(1 \mathrm{ml}, 0.2 \mathrm{M})$, and irradiated with blue light for $24 \mathrm{~h}$. The crude mixture was purified by silica gel chromatography (gradient hexane to hexaneEtOAc 99:1) yielding a white solid. This residue was extracted with pentane, the insoluble solid (triphenylpyridine) was removed by decantation and the liquid extract was concentrated in vacuo yielding a white solid $(20 \mathrm{mg})$. This material contains a mixture of triphenylpyridine and 3an which we were unable to separate further. According to ${ }^{1} \mathrm{H}$ NMR the mixture contains $12 \mathrm{mg}(20 \%)$ of $3 \mathbf{a n}$.

${ }^{1} \mathbf{H}$ NMR $\left(400 \mathrm{MHz}, \mathrm{CDCl}_{3}\right) \delta 7.36-7.24(\mathrm{~m}, 2 \mathrm{H}), 7.00(\mathrm{~d}, J=7.4 \mathrm{~Hz}, 2 \mathrm{H}), 6.91(\mathrm{dd}, J=$ $8.2,6.6 \mathrm{~Hz}, 1 \mathrm{H}), 6.83-6.87(\mathrm{~m}, 2 \mathrm{H}), 6.47(\mathrm{dd}, J=16.0,1.1 \mathrm{~Hz}, 1 \mathrm{H}), 6.19$ (dd, $J=16.0,7.4$ $\mathrm{Hz}, 1 \mathrm{H}), 3.81(\mathrm{~s}, 3 \mathrm{H}), 3.71(\mathrm{~m}, 2 \mathrm{H}), 2.84(\mathrm{~m}, 1 \mathrm{H}), 2.29(\mathrm{~s}, 6 \mathrm{H}), 1.29(\mathrm{~d}, J=6.8 \mathrm{~Hz}, 3 \mathrm{H})$.

${ }^{13} \mathbf{C}$ NMR $\left(100 \mathrm{MHz}, \mathrm{CDCl}_{3}\right)$ was not interpreted due to the overlapping signals of 3an with triphenylpyridine.

\subsection{Limitations - Unsuccessful examples}

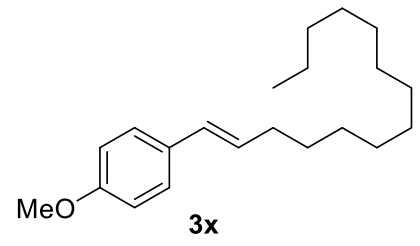

- $1 \mathrm{~b}$ and $6 \mathrm{~s}$ reacted at $42-46{ }^{\circ} \mathrm{C}$ - no product $3 \mathbf{x}$ formed by ${ }^{1} \mathrm{H}$ NMR

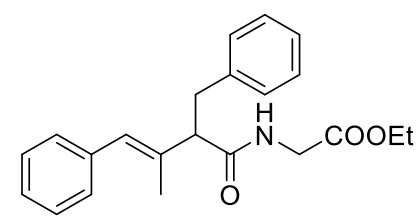

3ak

- $1 \mathrm{~m}$ and $6 \mathrm{t}$ reacted according to General procedure

- complex mixture obtained

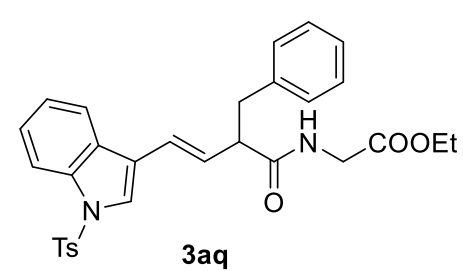

- $1 p$ and $6 t$ reacted according to General procedure - product 3aq isolated (22\%) but with insufficient purity

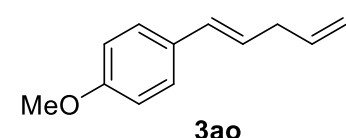

- $1 \mathrm{~b}$ and $6 \mathrm{u}$ reacted according to General procedure

- $12 \%$ NMR yield of 3 ao

- inseparable mixture

- volatile product

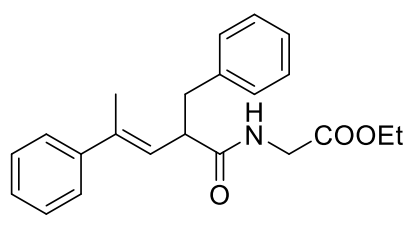

3al

- $1 \mathrm{n}$ and $6 \mathrm{t}$ reacted according to General procedure

- complex mixture obtained<smiles>CCOC(=O)CNC(=O)C(/C=C/c1ccc([N+](=O)[O-])cc1)Cc1ccccc1</smiles>

- $1 \mathrm{q}$ and $6 \mathrm{t}$ reacted according to General procedure

- no product 3ar formed according to ${ }^{1} \mathrm{H}$ NMR

- rapid polymerisation of nitrostyrene<smiles>COc1ccc(/C=C/CCc2ccccc2)cc1</smiles>

- $1 \mathrm{~b}$ and $6 \mathrm{v}$ reacted according to General procedure - complex mixture formed<smiles>CCC/C=C/C(Cc1ccccc1)C(=O)NCC(=O)OCC</smiles>

3am

- 10 and $6 \mathrm{t}$ reacted according to General procedure

- complex mixture formed<smiles>CCOC(=O)CNC(=O)C(/C=C/c1ccc(C#N)cc1)Cc1ccccc1</smiles>

- $1 r$ and $6 t$ reacted according to General procedure

- no product 3 as formed according to ${ }^{1} \mathrm{H}$ NMR

- rapid polymerisation of nitrostyrene 


\section{References}

1. Venkatanna, K.; Kumar, S. Y.; Karthick, M.; Padmanaban, R.; Ramanathan, C. R. Org. Biomol. Chem. 2019, 17, 4077-4086.

2. Zheng, C.; Huang, S.; Liu, Y.; Jiang, C.; Zhang, W.; Fang, G.; Hong, J. Org. Lett. 2020, 12, 4868-4872.

3. Maity, S.; Manna, S.; Rana, S.; Naveen, T.; Mallick A.; Maiti D. J. Am. Chem. Soc. 2013, 135, 3355-3358.

4. Trost, B. M.; Müller, C. J. Am. Chem. Soc. 2008, 130, 2438-2439.

5. $\quad$ Kinsman, A. C.; Kerr, M. A. Org. Lett. 2001, 3, 3189-3191.

6. Klauck, F. J.; Yoon, H.; James, M. J.; Lautens, M.; Glorius, F. ACS Catal. 2019, 9, 236-241.

7. Ociepa, M.; Turkowska, J.; Gryko, D. ACS Catal. 2018, 8, 11362-11367.

8. Huang, Y.; Jia, J.; Huang, Q. P.; Zhao, L.; Wang, P.; Gu, J.; He, C. Y. Chem. Commun. 2020, 56, 14247-14250.

9. James, M. J.; Strieth-Kalthoff, F.; Sandfort, F.; Klauck, F. J.; Wagener, F.; Glorius, F. Chem. Eur. J. 2019, 25, 8240-8244.

10. Hoerner, M. E.; Baker, K. M.; Basch, C. H.; Bampo, E. M.; Watson, M. P. Org. Lett. 2019, 21, 7356-7360.

11. Basch, C. H.; Liao, J.; Xu, J.; Piane, J. J.; Watson, M. P. J. Am. Chem. Soc. 2017, 139, 5313-5316.

12. Klauck, F. J.; James, M. J.; Glorius, F. Angew. Chem., Int. Ed. 2017, 56, 12336-12339.

13. Sun, S. Z.; Romano, C.; Martin, R. J. Am. Chem. Soc. 2019, 141, 16197-16201.

14. Plunkett, S. B.; Corey H.; Santana, S. O.; Watson, M. P. J. Am. Chem. Soc. 2019, 141, 2257-2262.

15. Dominguez, X. A.; Slim, S. J.; Elizondo, A. J. Am. Chem. Soc. 1953, 75, 4581-4582.

16. King; W. J; Nord, F. F. J. Org. Chem. 1949, 14, 405-409.

17. M. Correia, J. T.; A. Fernandes, V.; Matsuo, B. T.; C. Delgado, J. A.; de Souza, W. C.; Paixão, M. W. Chem. Commun. 2020, 56, 503-514.

18. Grimshaw, J.; Moore, S.; Trocha-Grimshaw, J. Acta Chem. Scand. Ser. B 1983, 37, 485.

19. Martínez-Gualda, A. M.; Cano, R.; Marzo, L.; Pérez-Ruiz, R.; Luis-Barrera, J.; MasBallesté, R.; Fraile, A.; A. de la Peña O’Shea, V.; Alemán, J. Nat. Commun. 2019, 10, 2634.

20. Yang, H.; Sun, P.; Zhu, Y.; Yan, H.; Lu, L.; Qu, X.; Li, T.; Mao, J. Chem. Commun. 2012, 48, 7847-7849.

21. Liu, Q.; Yi, H.; Liu, J.; Yang, Y.; Zhang, X.; Zeng, Z.; Lei, A. Chem. Eur. J. 2013, 19, 5120-5126.

22. Li, G.; Wu, L; Lv, G.; Liu, H.; Fu, Q.; Zhang, X.; Tang, Z.; Chem. Commun. 2014, 50, 6246-6248.

23. Koy, M.; Sandfort, F.; Tlahuext-Aca, A.; Quach, L.; Daniliuc, C. G.; Glorius, F. Chem. Eur. J. 2018, 24, 4552-4555. 


\section{4. ${ }^{1} \mathrm{H},{ }^{13} \mathrm{C}$, and ${ }^{19} \mathrm{~F}$ NMR spectra}

\section{1. ${ }^{1} \mathrm{H}$ and ${ }^{13} \mathrm{C}$ NMR spectra of $1 \mathrm{~g}$}
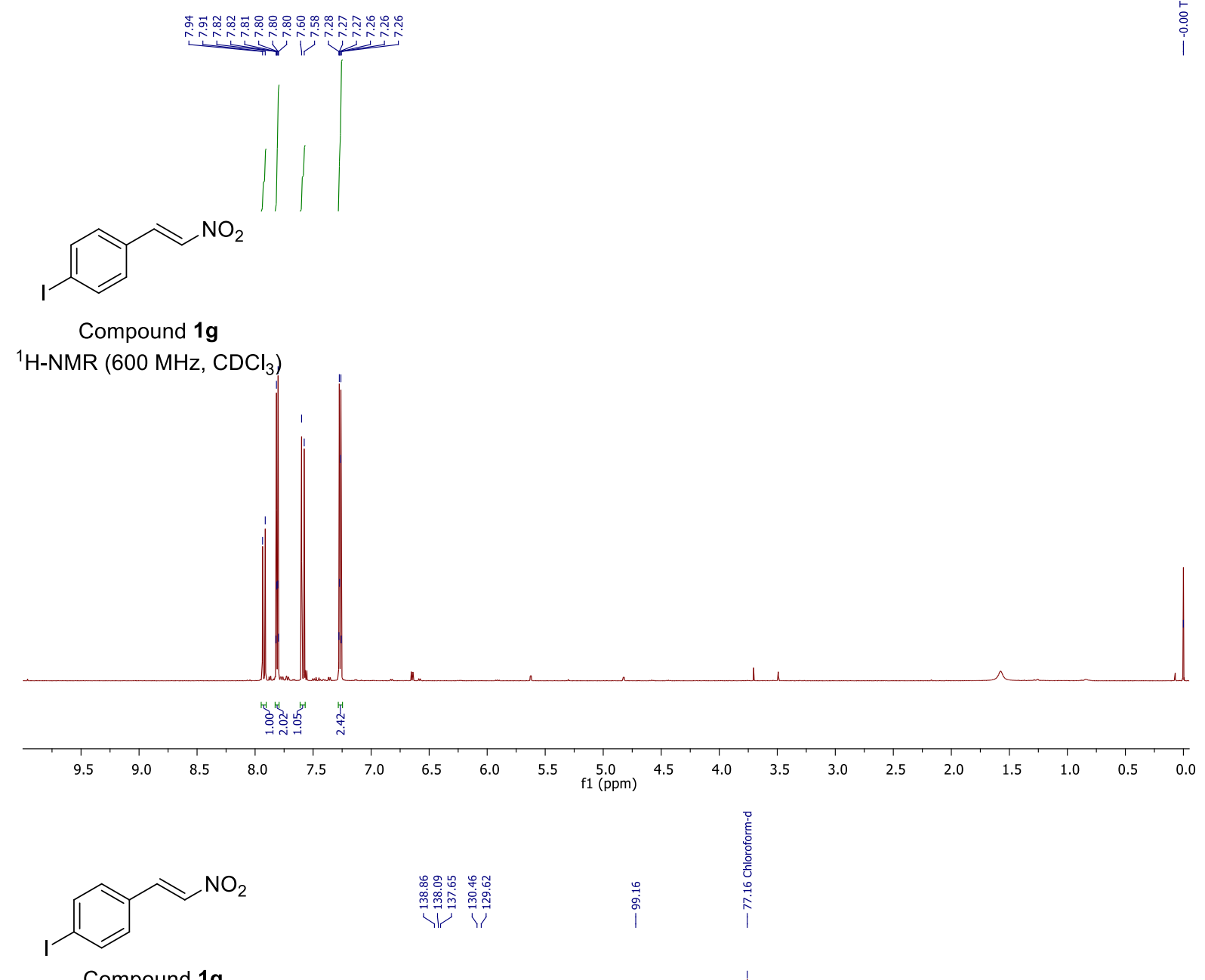

${ }^{13} \mathrm{C}-\mathrm{NMR}\left(151 \mathrm{MHz}, \mathrm{CDCl}_{3}\right)$

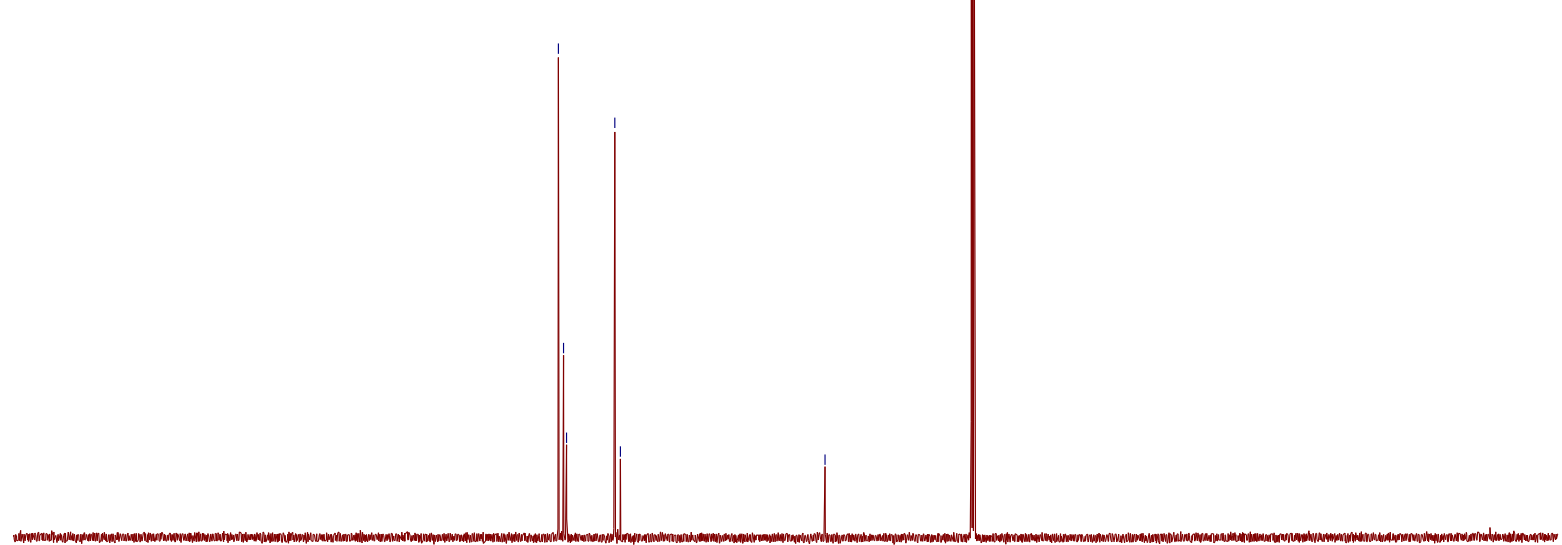

$\begin{array}{lllllllllll}210 & 200 & 190 & 180 & 170 & 160 & 150 & 140 & 130 & 120 & \begin{array}{c}110 \\ \mathrm{f} 1(\mathrm{ppm})\end{array}\end{array}$ 


\section{2. ${ }^{1} \mathrm{H}$ and ${ }^{13} \mathrm{C}$ NMR spectra of $1 \mathrm{~h}$}
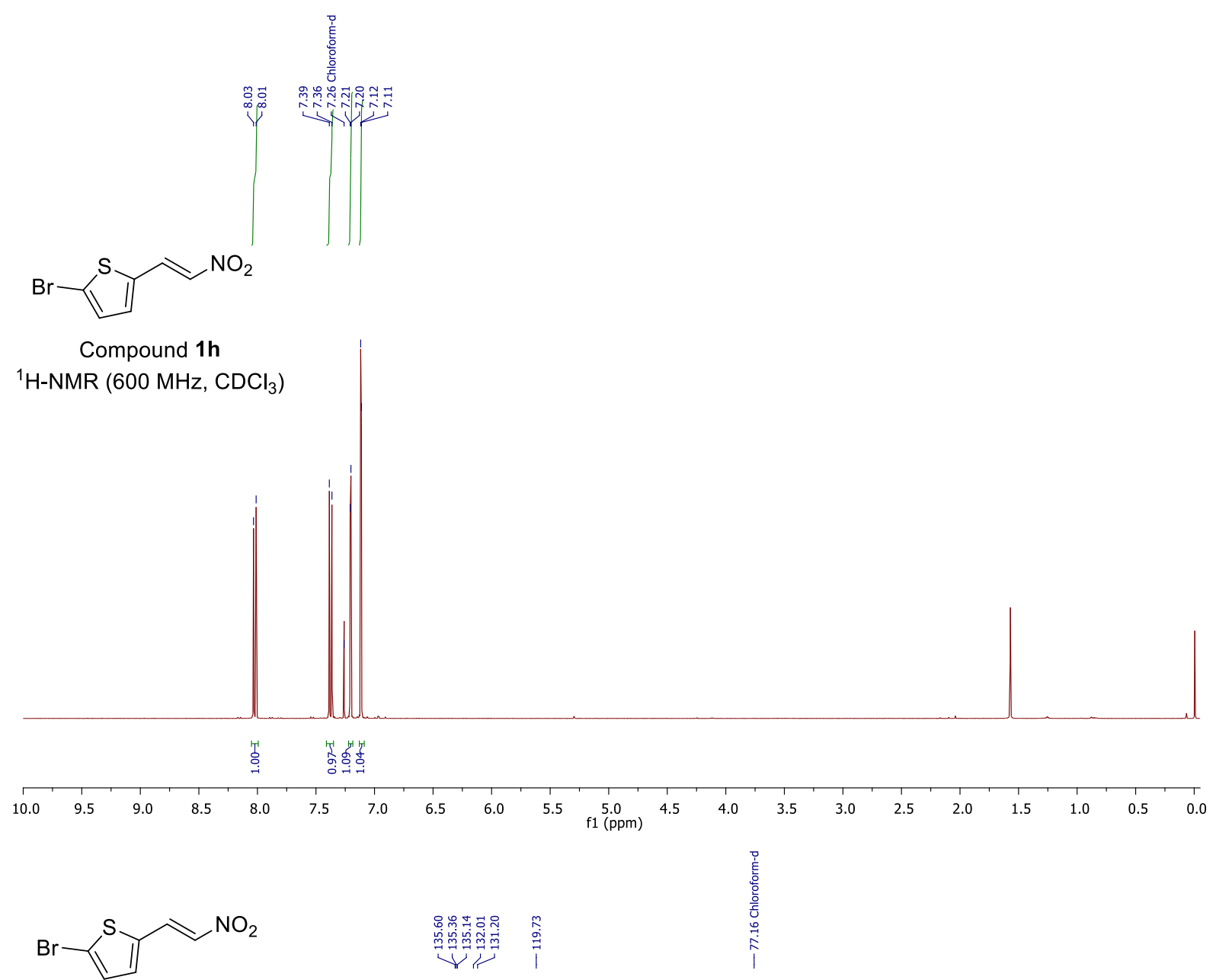

V|

Compound $1 \mathrm{~h}$

${ }^{13} \mathrm{C}-\mathrm{NMR}\left(151 \mathrm{MHz}, \mathrm{CDCl}_{3}\right.$ )

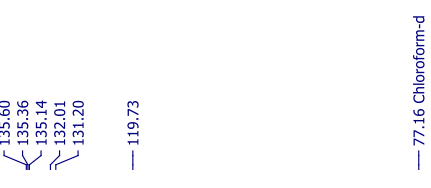
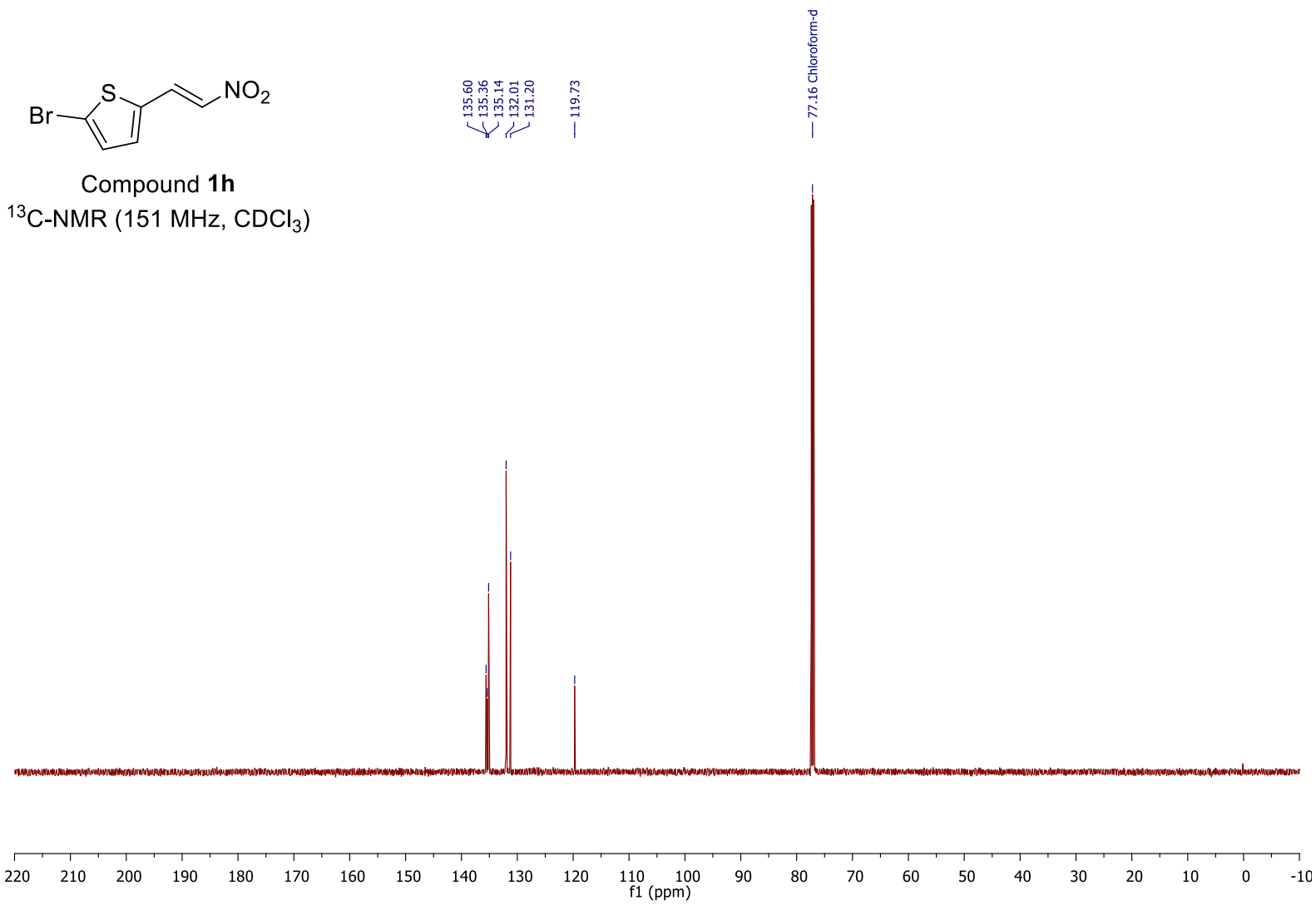


\section{3. ${ }^{1} \mathrm{H}$ and ${ }^{13} \mathrm{C}$ NMR spectra of $1 \mathrm{i}$}
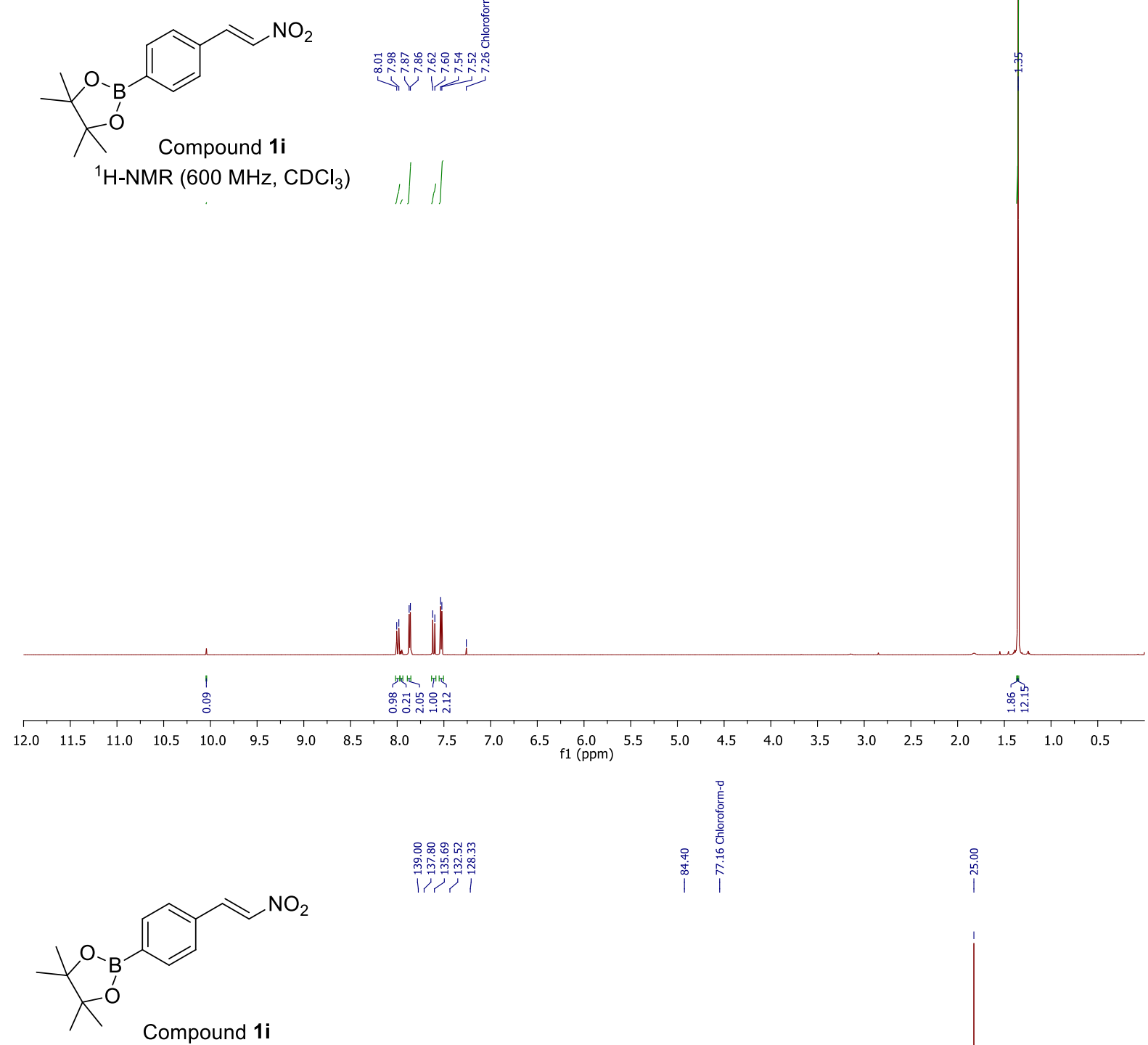

${ }^{13} \mathrm{C}-\mathrm{NMR}\left(151 \mathrm{MHz}, \mathrm{CDCl}_{3}\right.$ )

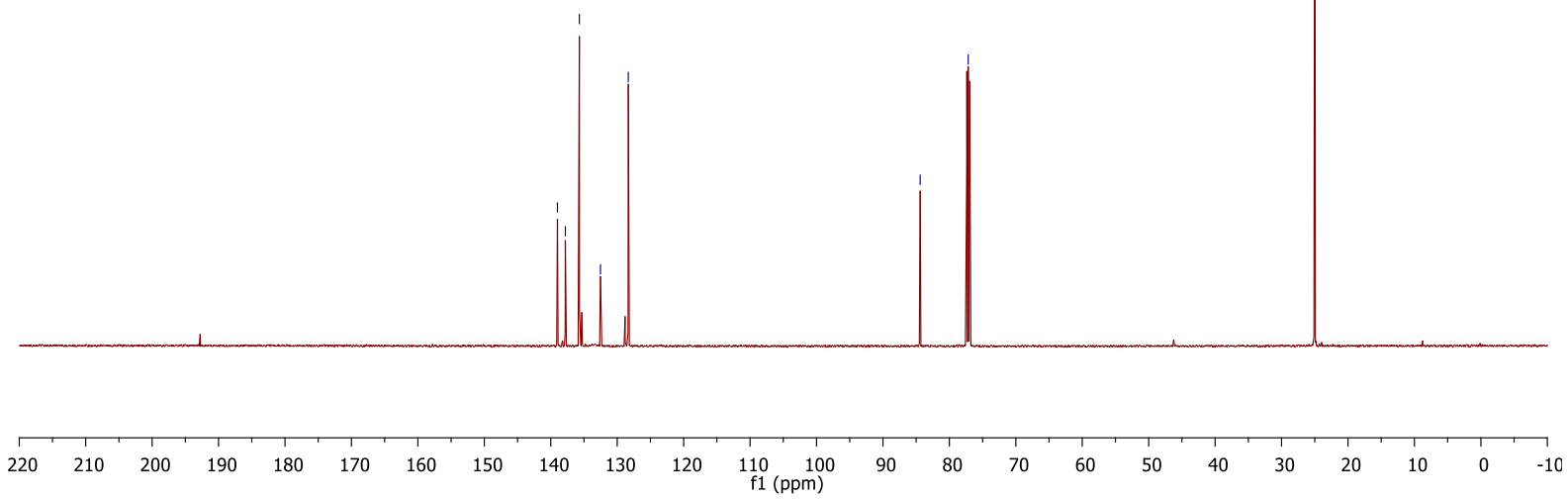




\section{4. ${ }^{1} \mathrm{H},{ }^{13} \mathrm{C}$, and ${ }^{19} \mathrm{~F}$ NMR spectra of $6 \mathrm{c}$}
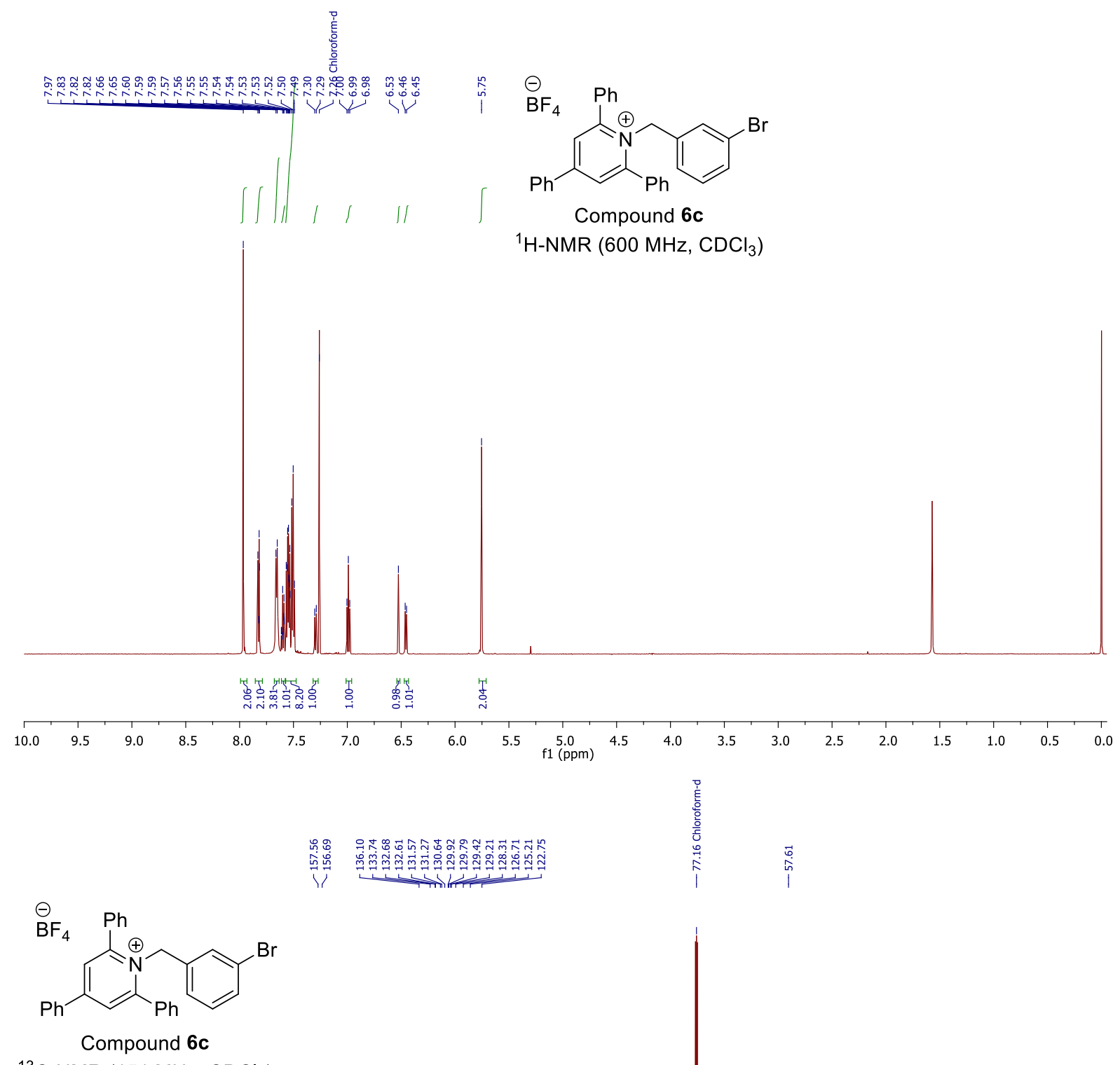

${ }^{13} \mathrm{C}-\mathrm{NMR}\left(151 \mathrm{MHz}, \mathrm{CDCl}_{3}\right)$

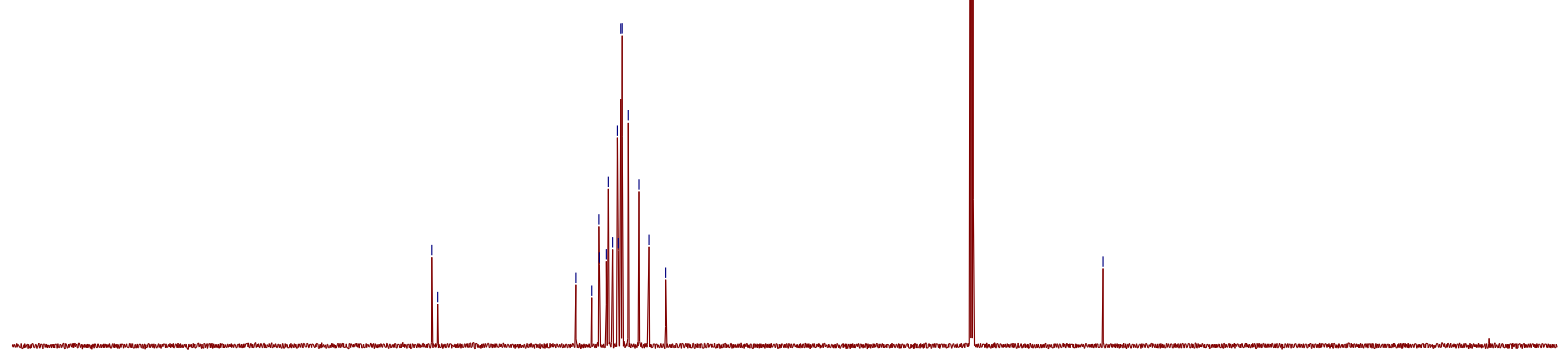

$\begin{array}{llllllllllllllllllllllll}220 & 210 & 200 & 190 & 180 & 170 & 160 & 150 & 140 & 130 & 120 & \begin{array}{c}110 \\ \mathrm{f} 1(\mathrm{ppm})\end{array} & 100 & 80 & 70 & 60 & 50 & 40 & 30 & 20 & 10 & 0 & -10\end{array}$ 
<smiles></smiles>

Compound $6 \mathrm{c}$

${ }^{19} \mathrm{~F}$-NMR $\left(564 \mathrm{MHz}, \mathrm{CDCl}_{3}\right.$ )

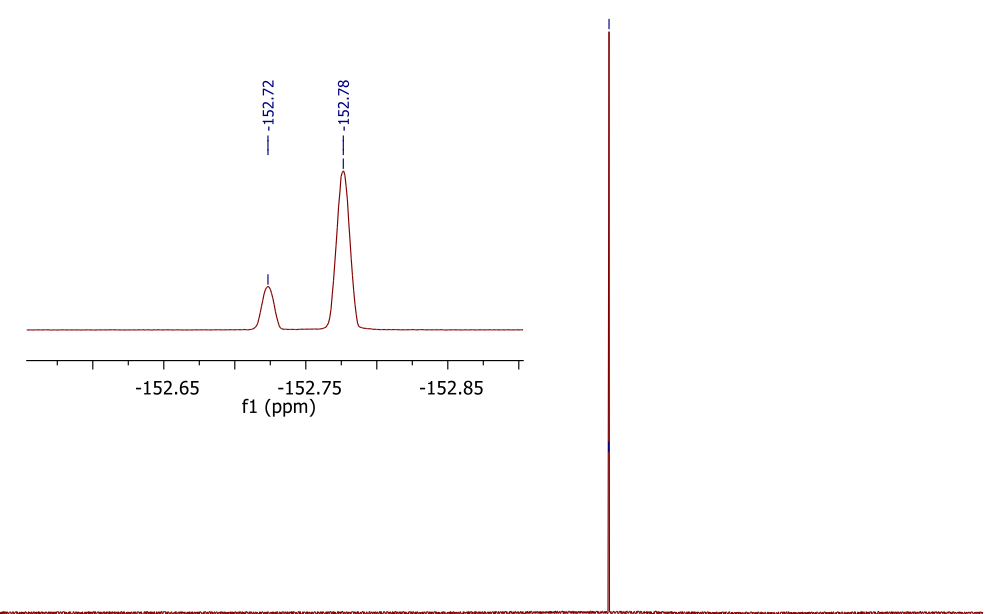

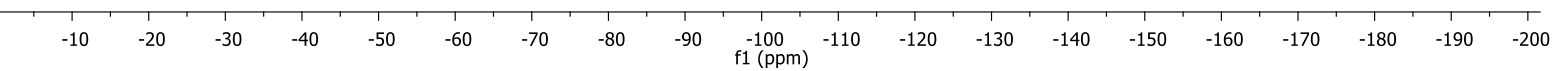




\section{5. ${ }^{1} \mathrm{H},{ }^{13} \mathrm{C}$, and ${ }^{19} \mathrm{~F}$ NMR spectra of $6 \mathrm{~s}$}
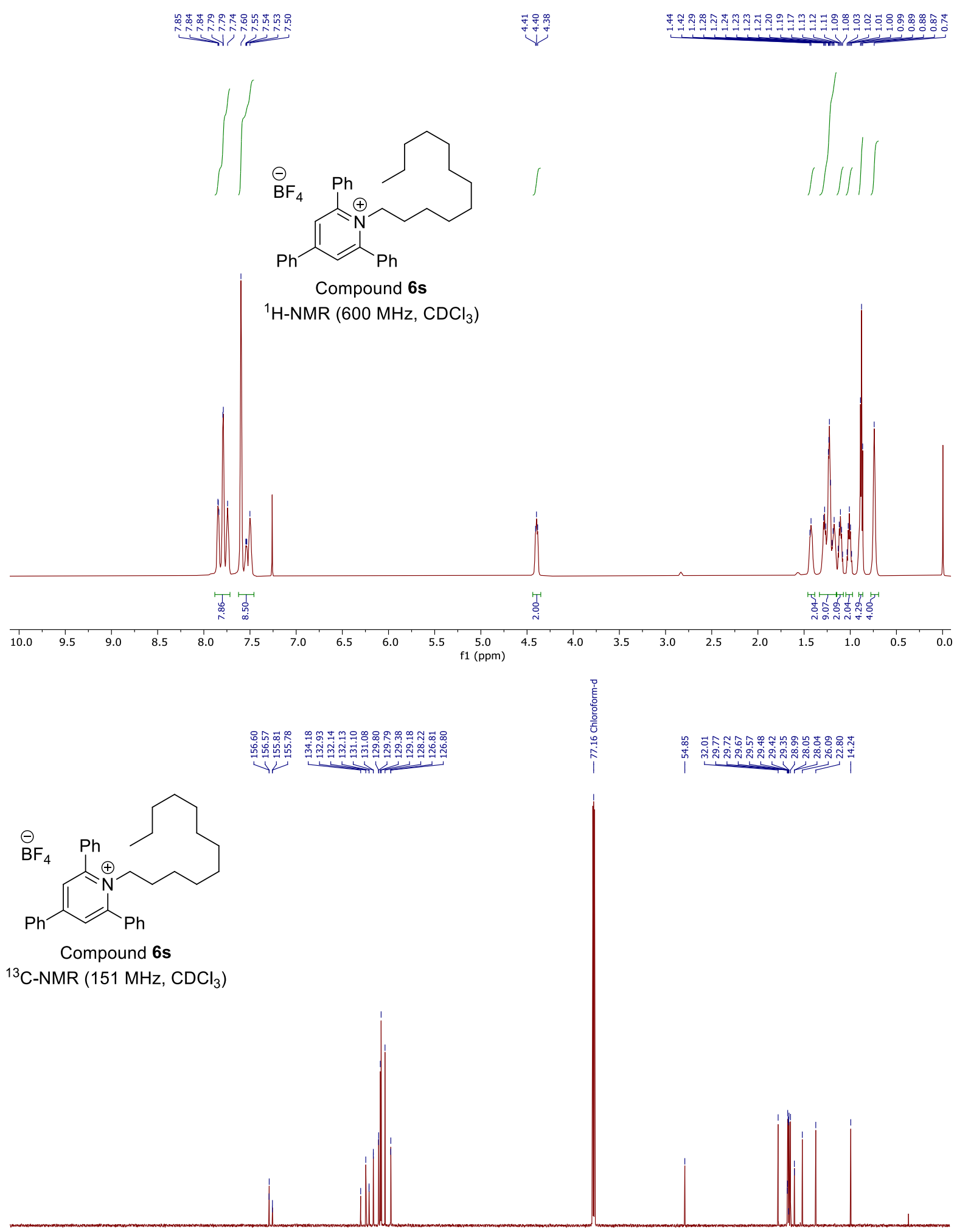

$\begin{array}{rlllllllllllllllllllllllllll}220 & 210 & 2 & 200 & 190 & 180 & 170 & 160 & 150 & 140 & 130 & 120 & \underset{f 1}{110}(\mathrm{ppm}) & 100 & 90 & 80 & 70 & 60 & 50 & 40 & 30 & 20 & 10 & 0 & -10\end{array}$ 


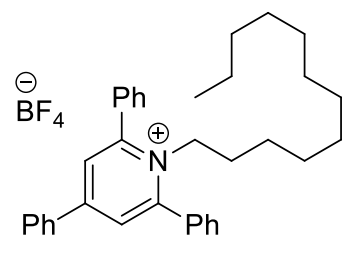

Compound $\mathbf{6 s}$

${ }^{19} \mathrm{~F}-\mathrm{NMR}\left(564 \mathrm{MHz}, \mathrm{CDCl}_{3}\right)$

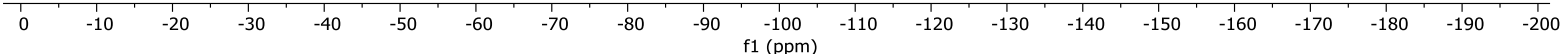




\section{6. ${ }^{1} \mathrm{H}$ and ${ }^{13} \mathrm{C}$ NMR spectra of $3 f$}
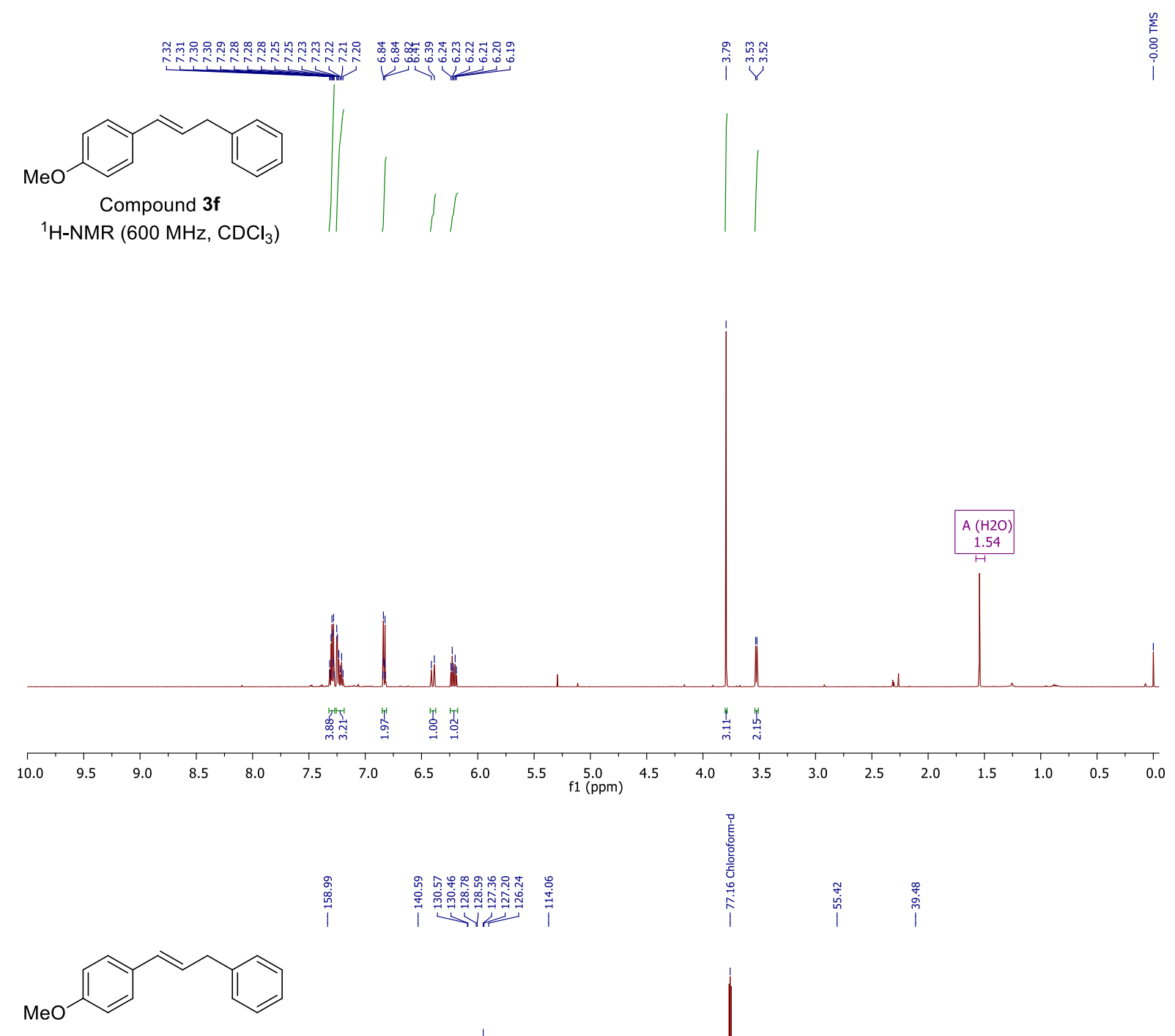

Compound $3 \mathbf{f}$

${ }^{13} \mathrm{C}-\mathrm{NMR}\left(151 \mathrm{MHz}, \mathrm{CDCl}_{3}\right)$

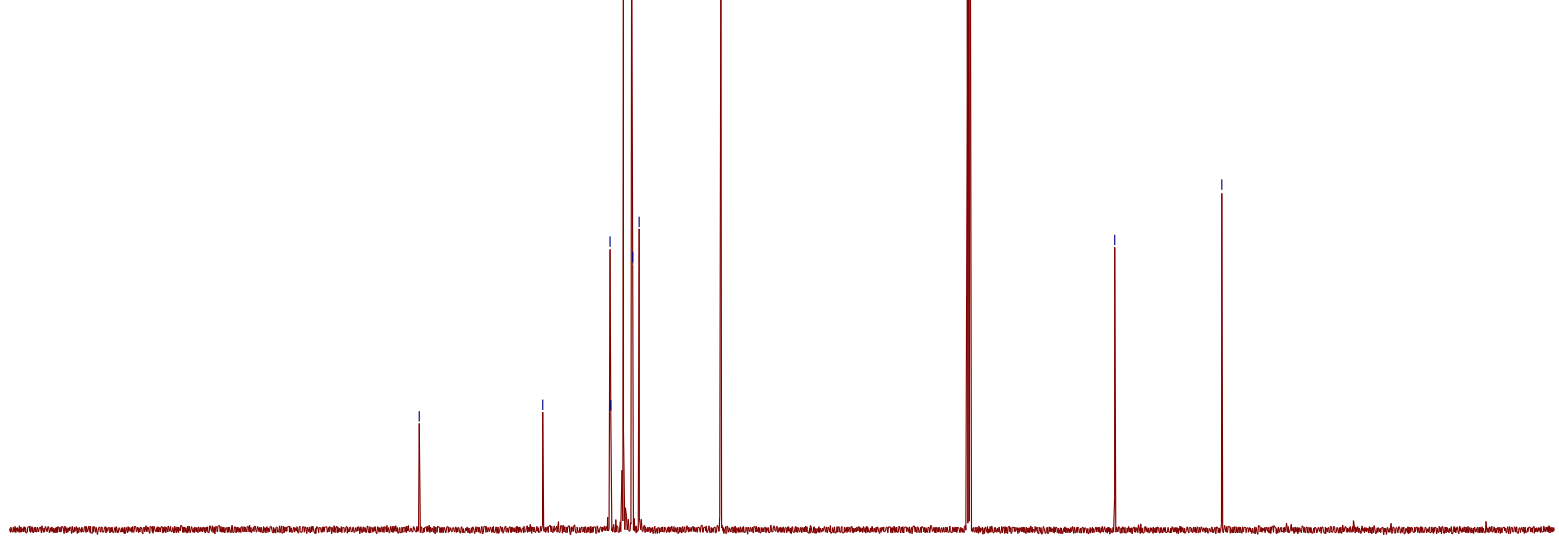

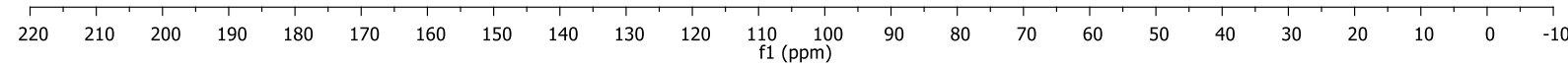




\section{7. ${ }^{1} \mathrm{H}$ and ${ }^{13} \mathrm{C}$ NMR spectra of $3 \mathrm{~g}$}
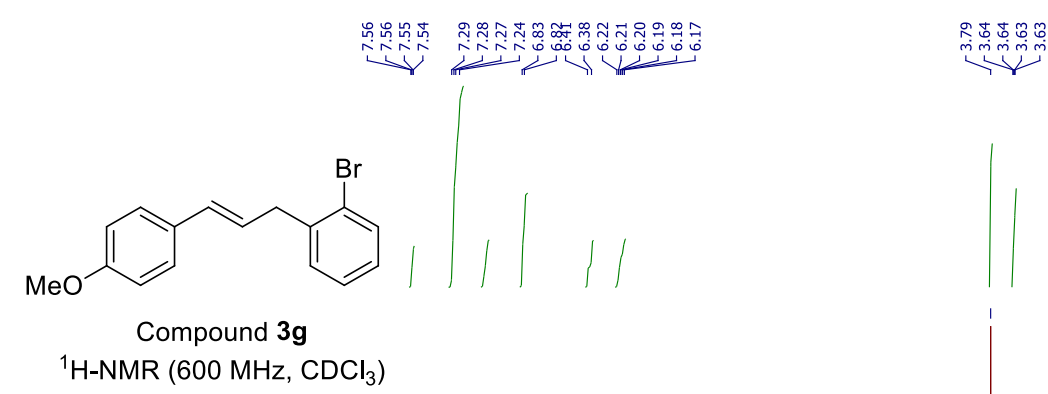

${ }^{1} \mathrm{H}-\mathrm{NMR}\left(600 \mathrm{MHz}, \mathrm{CDCl}_{3}\right)$
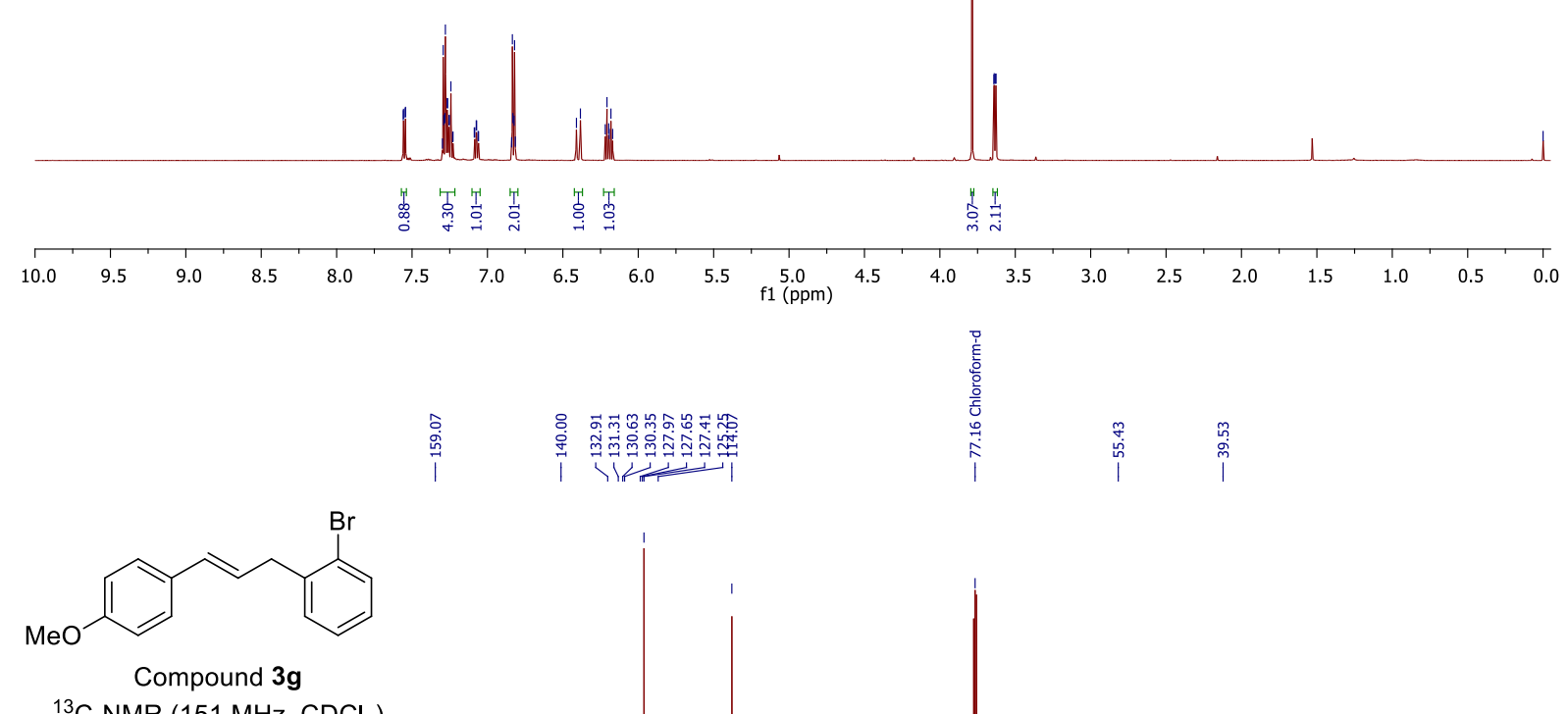

${ }^{13} \mathrm{C}-\mathrm{NMR}\left(151 \mathrm{MHz}, \mathrm{CDCl}_{3}\right)$

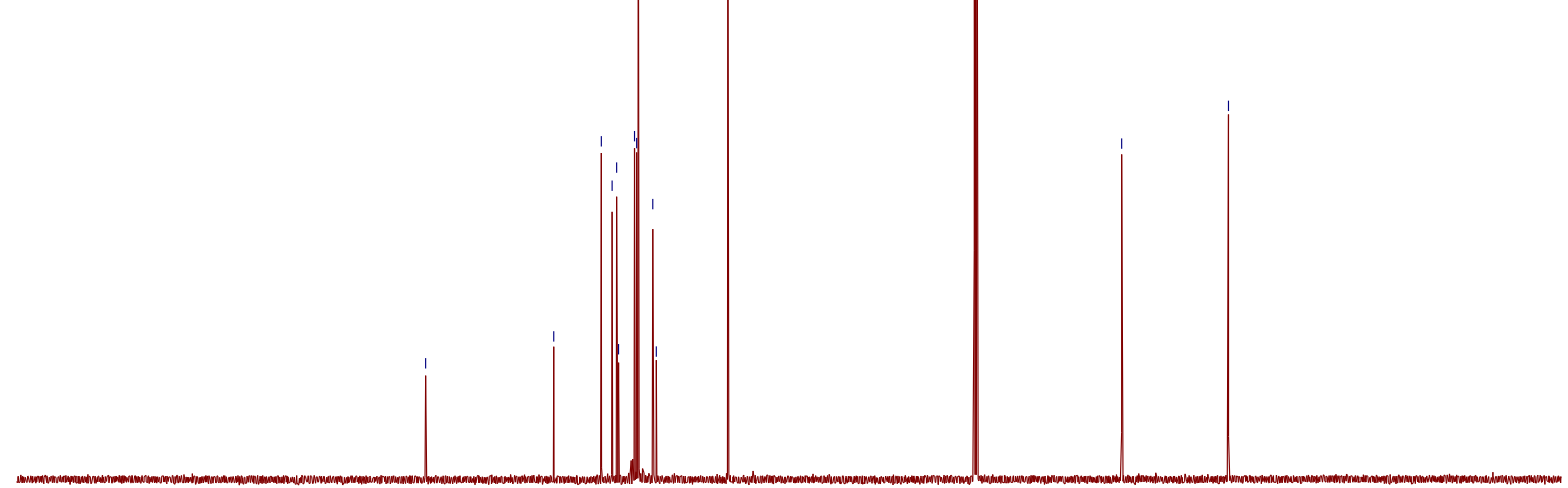

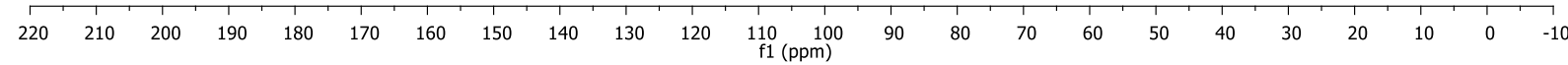




\section{8. ${ }^{1} \mathrm{H}$ and ${ }^{13} \mathrm{C}$ NMR spectra of $3 \mathrm{~h}$}
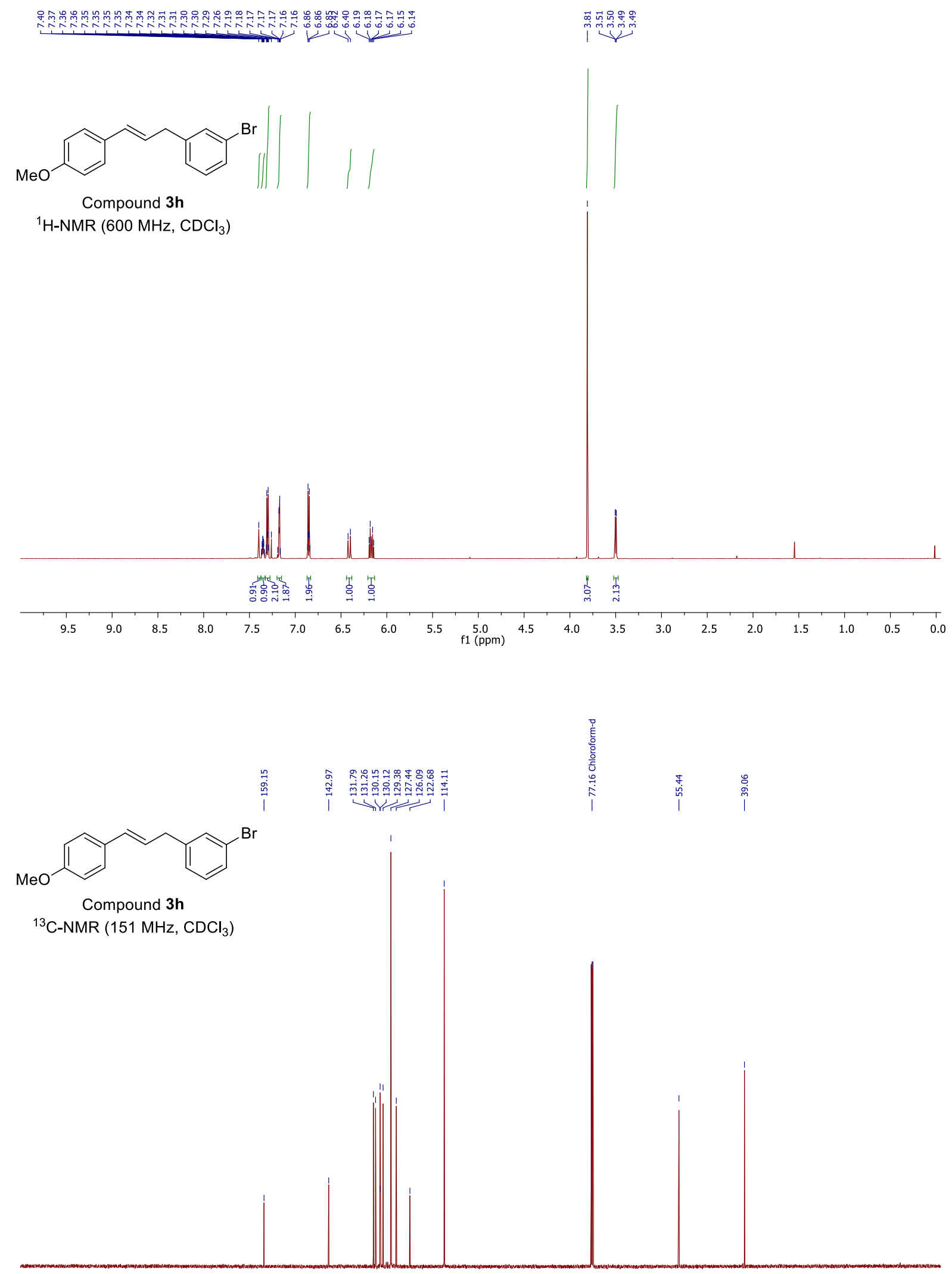

\begin{tabular}{rllllllllllllllllllllllll}
\hline 220 & 210 & 200 & 190 & 180 & 170 & 160 & 150 & 140 & 130 & 120 & 110 & 100 & 90 & 80 & 70 & 60 & 50 & 40 & 30 & 20 & 10 & 0 & -10
\end{tabular} 


\section{9. ${ }^{1} \mathrm{H}$ and ${ }^{13} \mathrm{C}$ NMR spectra of $3 i$}
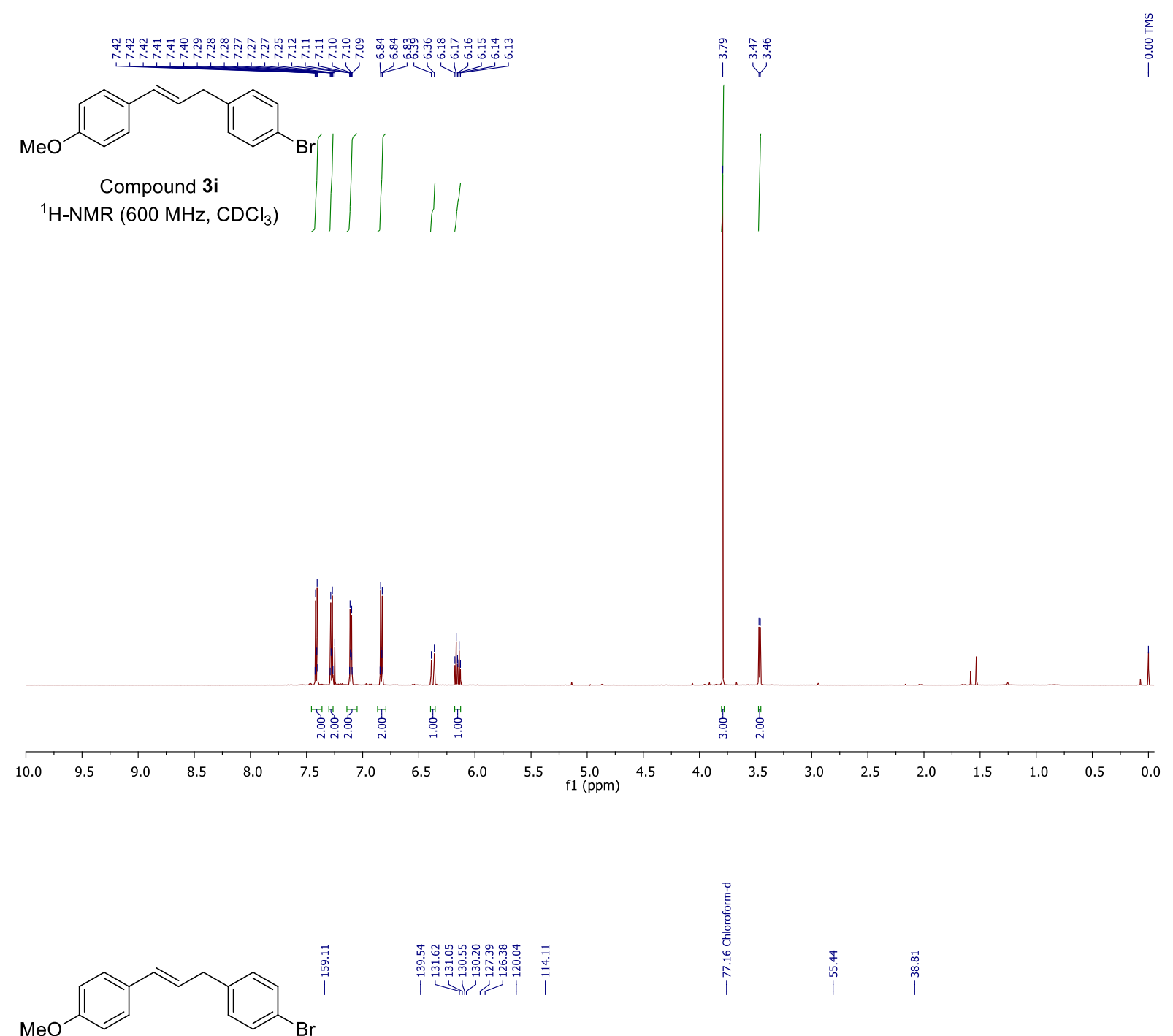

Compound $\mathbf{3 i}$

${ }^{13} \mathrm{C}-\mathrm{NMR}\left(151 \mathrm{MHz}, \mathrm{CDCl}_{3}\right)$

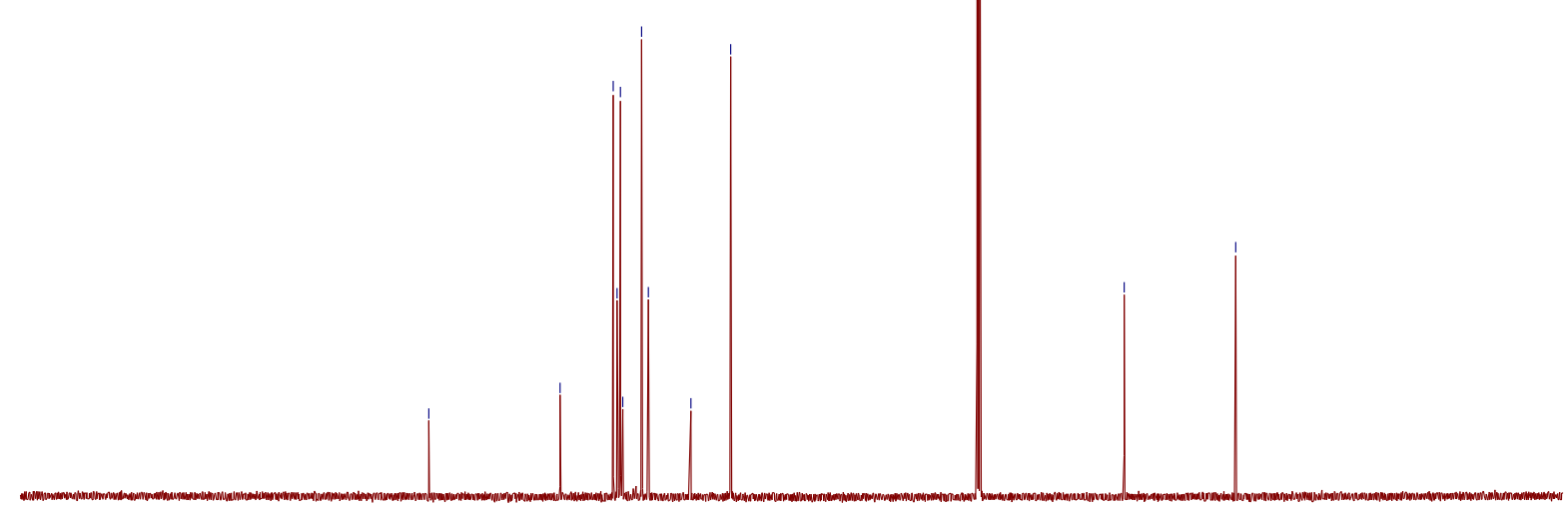

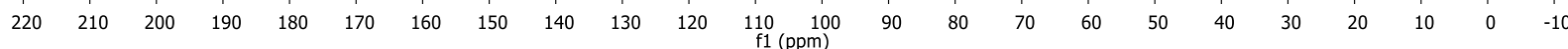




\subsection{0. ${ }^{1} \mathrm{H},{ }^{13} \mathrm{C}$, and ${ }^{19} \mathrm{~F}$ NMR spectra of $3 \mathrm{j}$}
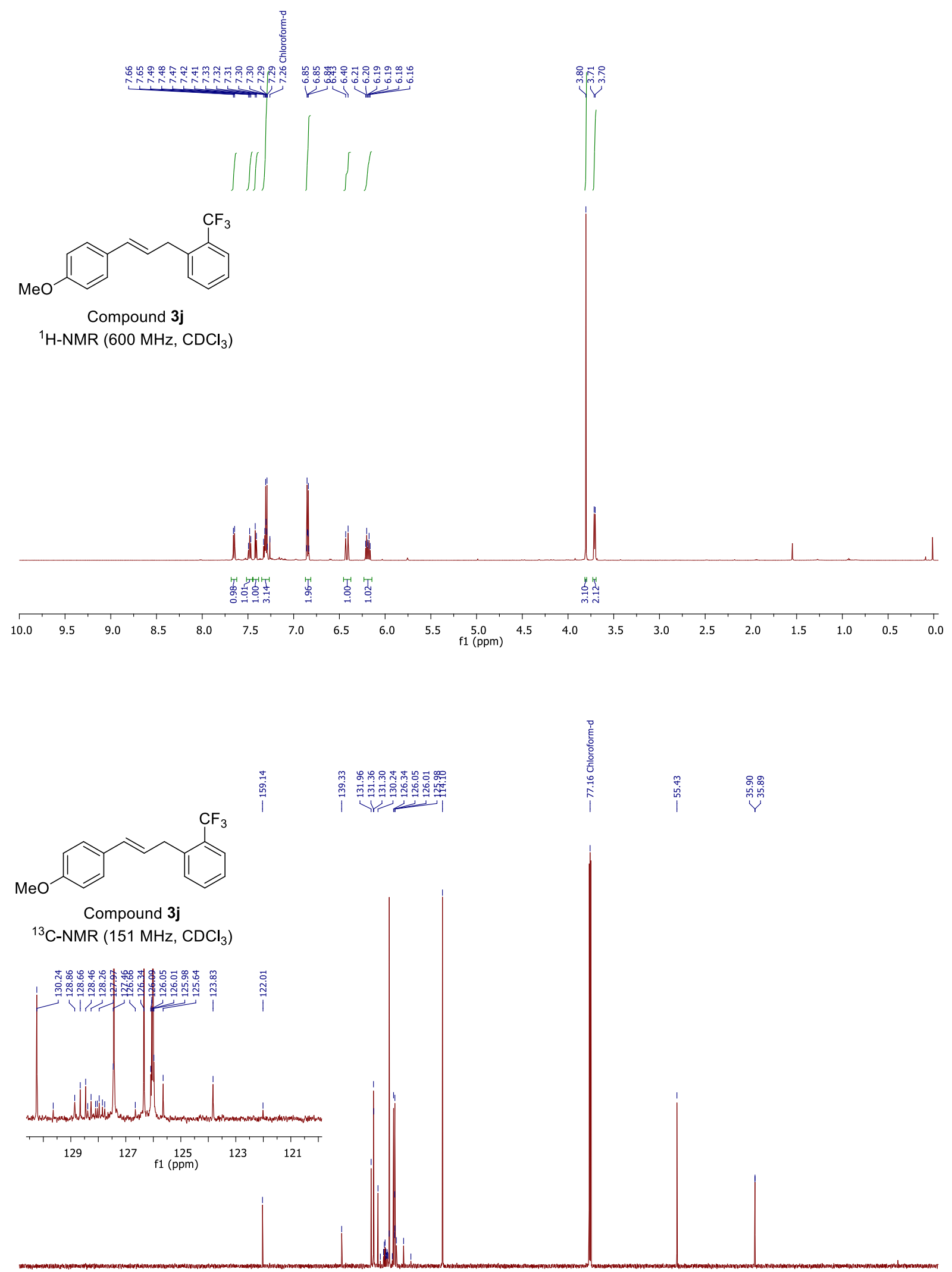

$\begin{array}{llllllllllllllllllllllllllll}220 & 210 & 200 & 190 & 180 & 170 & 160 & 150 & 140 & 130 & 120 & 110 & 100 & 90 & 80 & 70 & 60 & 50 & 40 & 30 & 20 & 10 & 0 & -10\end{array}$ 


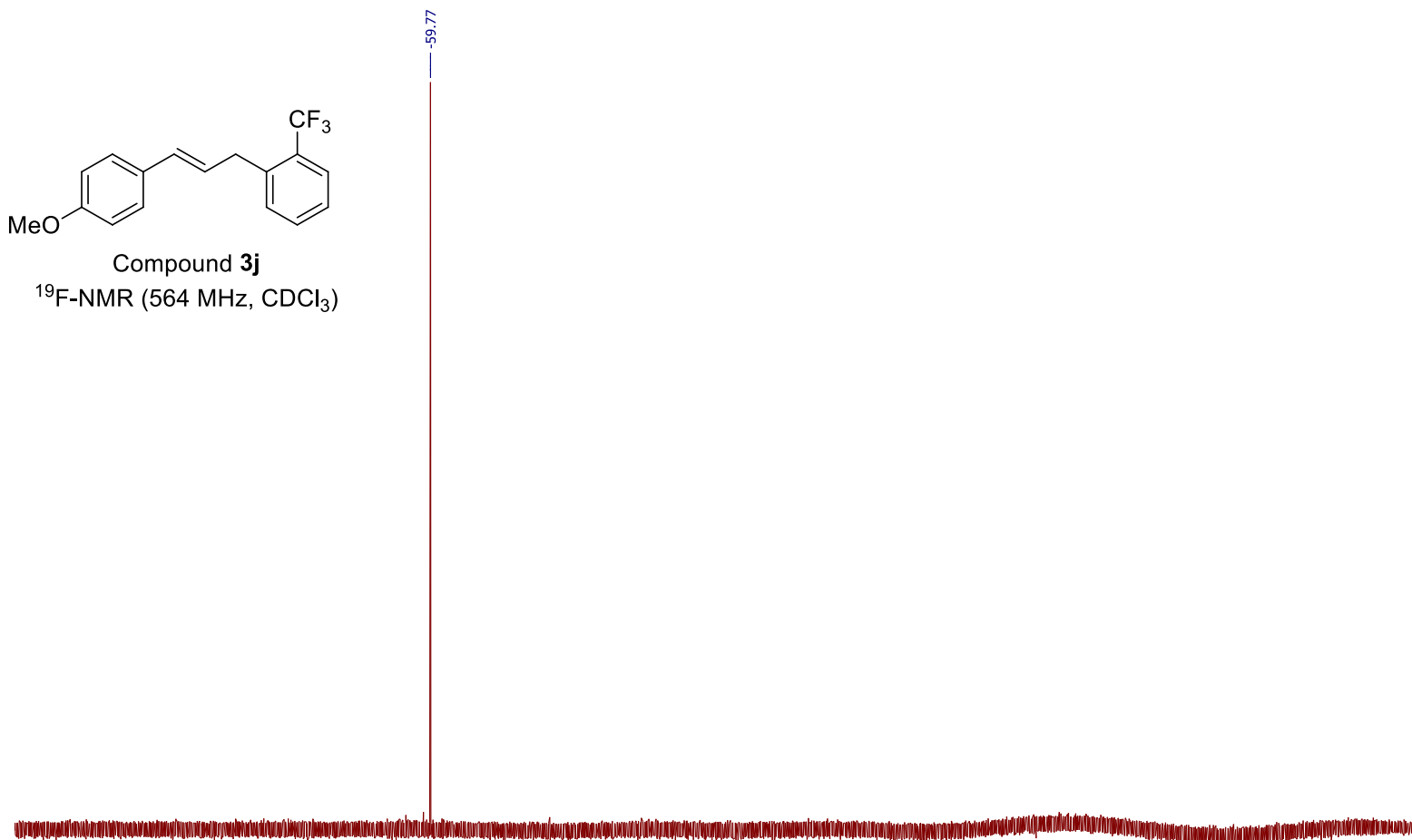

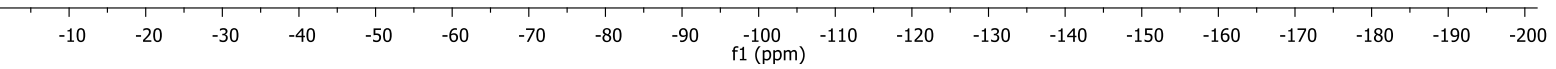




\subsection{1. ${ }^{1} \mathrm{H},{ }^{13} \mathrm{C}$, and ${ }^{19} \mathrm{~F}$ NMR spectra of $3 \mathrm{k}$}
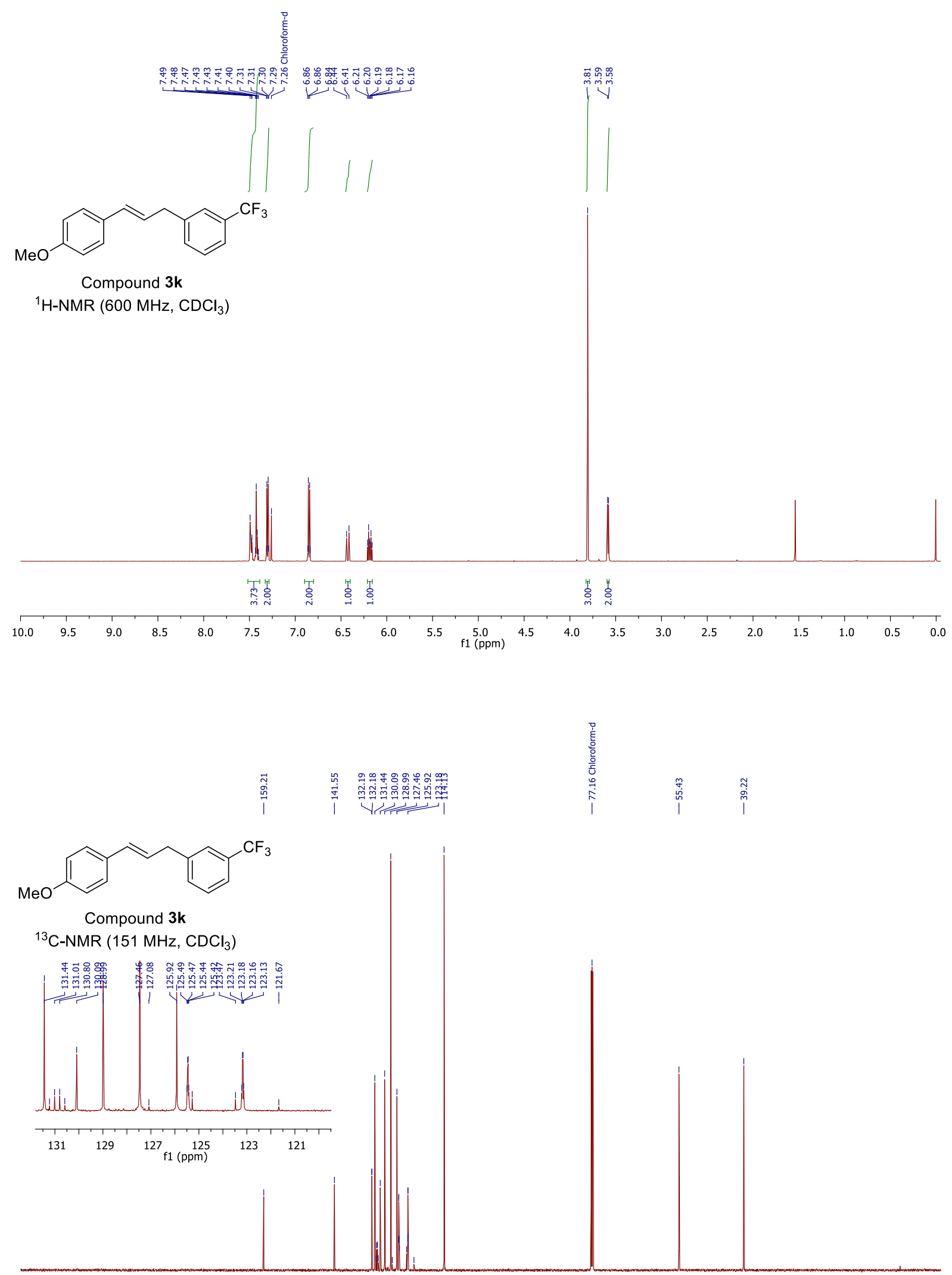

$\begin{array}{llllllllllllllllllllllllllll}220 & 210 & 200 & 190 & 180 & 170 & 160 & 150 & 140 & 130 & 120 & 110 & 100 & 90 & 80 & 70 & 60 & 50 & 40 & 30 & 20 & 10 & 0 & -10\end{array}$ 
<smiles>COc1ccc(/C=C/Cc2cccc(C(F)(F)F)c2)cc1</smiles>

${ }^{19}$ F-NMR (564 MHz, $\mathrm{CDCl}_{3}$ )

$\begin{array}{llllllllllllllllllll}-10 & -20 & -30 & -40 & -50 & -60 & -70 & -80 & -90 & -100 & -110 & -120 & -130 & -140 & -150 & -160 & -170 & -180 & -190 & -200\end{array}$




\subsection{2. ${ }^{1} \mathrm{H},{ }^{13} \mathrm{C}$, and ${ }^{19} \mathrm{~F}$ NMR spectra of $3 \mathrm{I}$}
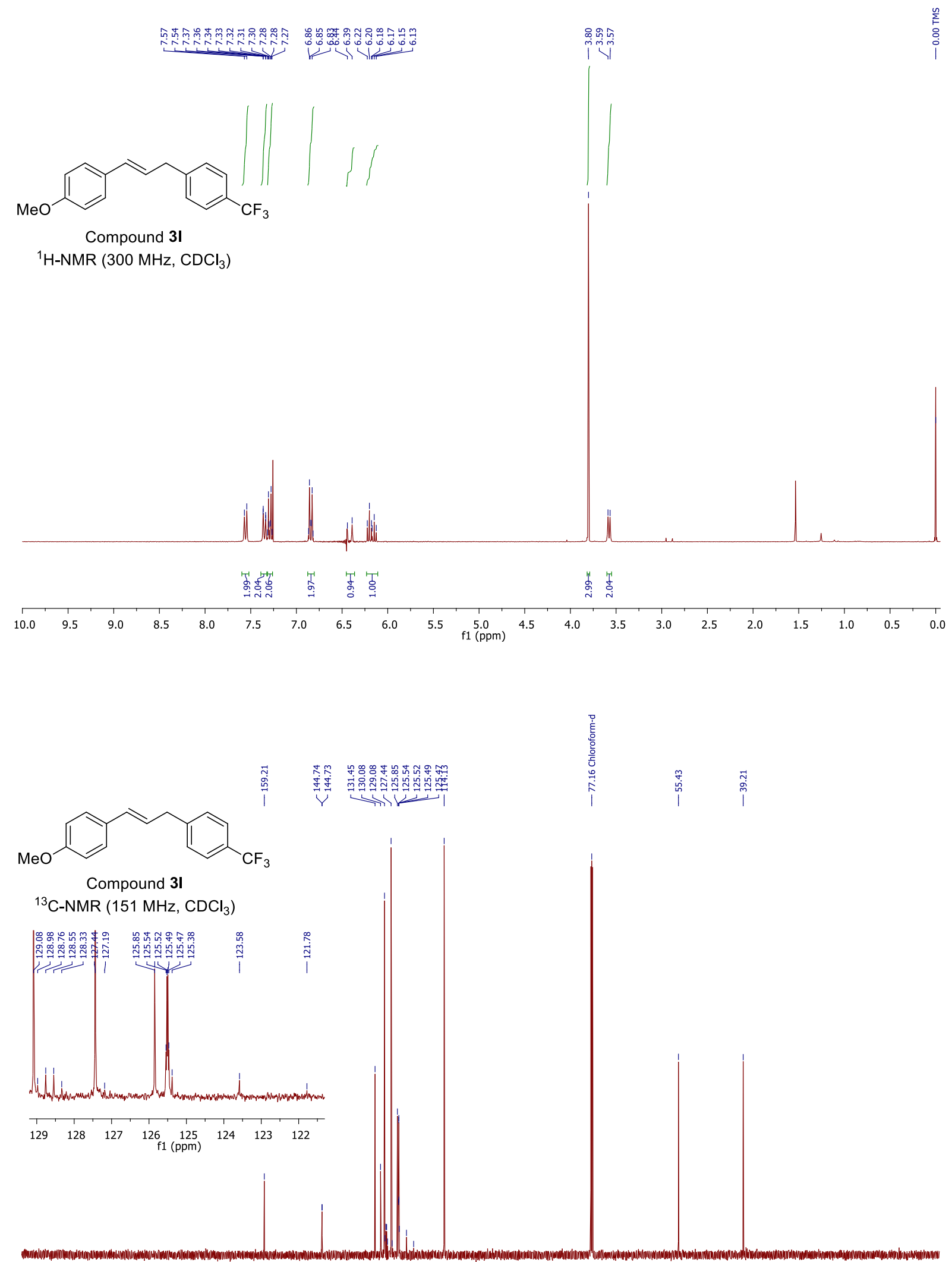

$\begin{array}{llllllllllll}210 & 200 & 190 & 180 & 170 & 160 & 150 & 140 & 130 & 120 & \begin{array}{c}110 \\ \mathrm{f} 1(\mathrm{ppm})\end{array}\end{array}$ 


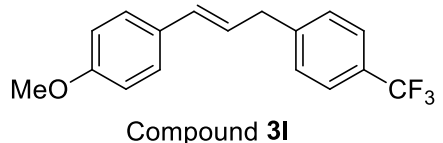

${ }^{19} \mathrm{~F}$-NMR $\left(564 \mathrm{MHz}, \mathrm{CDCl}_{3}\right.$ )

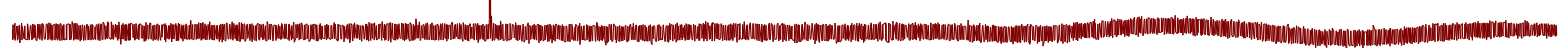

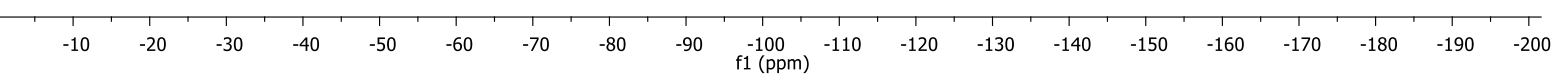




\subsection{3. ${ }^{1} \mathrm{H}$ and ${ }^{13} \mathrm{C}$ NMR spectra of $3 \mathrm{~m}$}

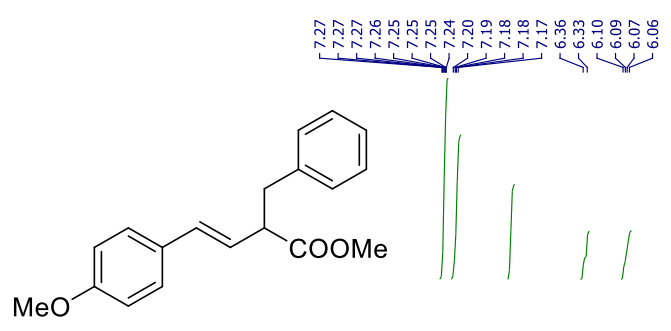

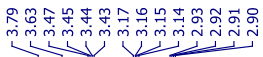

Compound $3 \mathrm{~m}$

${ }^{1} \mathrm{H}-\mathrm{NMR}\left(600 \mathrm{MHz}, \mathrm{CDCl}_{3}\right)$
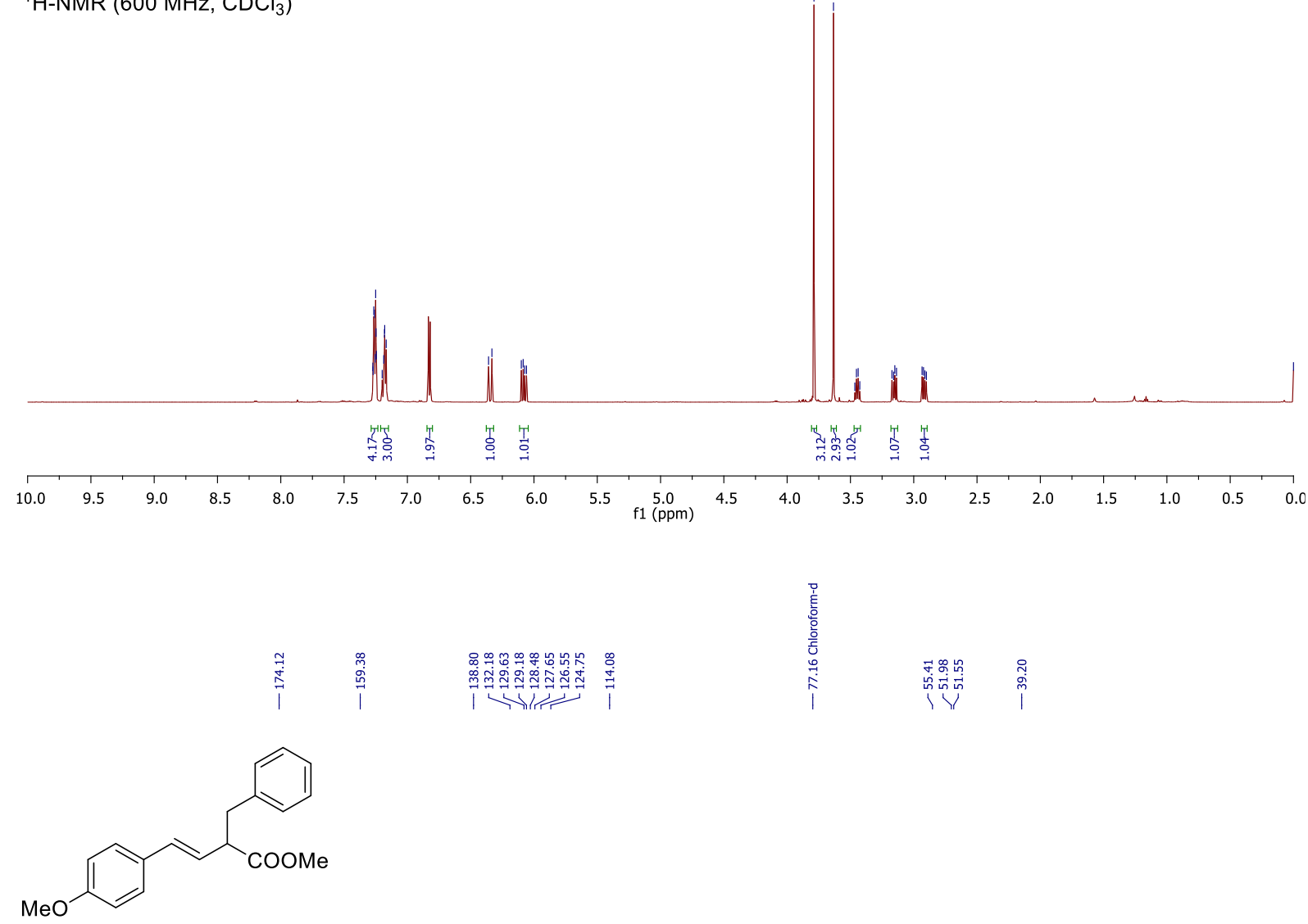

Compound 3m

${ }^{13} \mathrm{C}-\mathrm{NMR}\left(151 \mathrm{MHz}, \mathrm{CDCl}_{3}\right)$

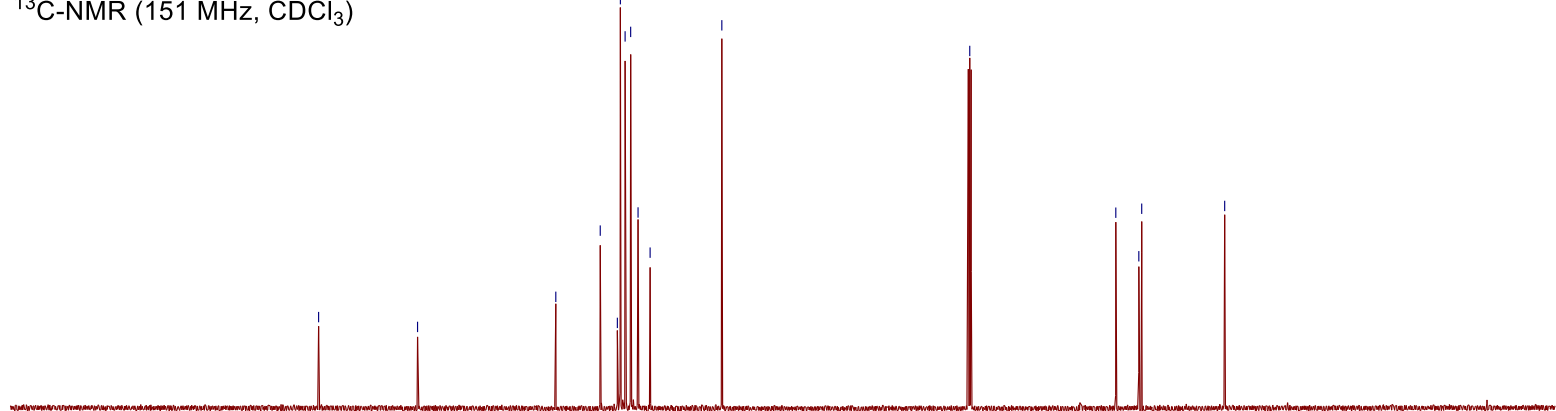

$\begin{array}{llllllllllllllllllllllllllllllll}220 & 210 & 200 & 190 & 180 & 170 & 160 & 150 & 140 & 130 & 120 & 110 & 100 & 90 & 80 & 70 & 60 & 50 & 40 & 30 & 20 & 10 & 0 & -10\end{array}$ 


\subsection{4. ${ }^{1} \mathrm{H}$ and ${ }^{13} \mathrm{C}$ NMR spectra of $3 n$}
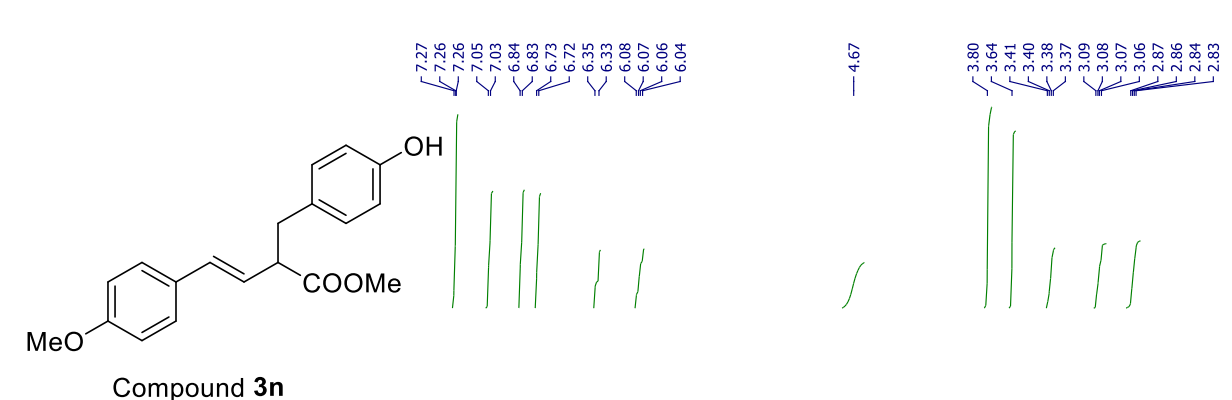

${ }^{1} \mathrm{H}-\mathrm{NMR}\left(600 \mathrm{MHz}, \mathrm{CDCl}_{3}\right)$
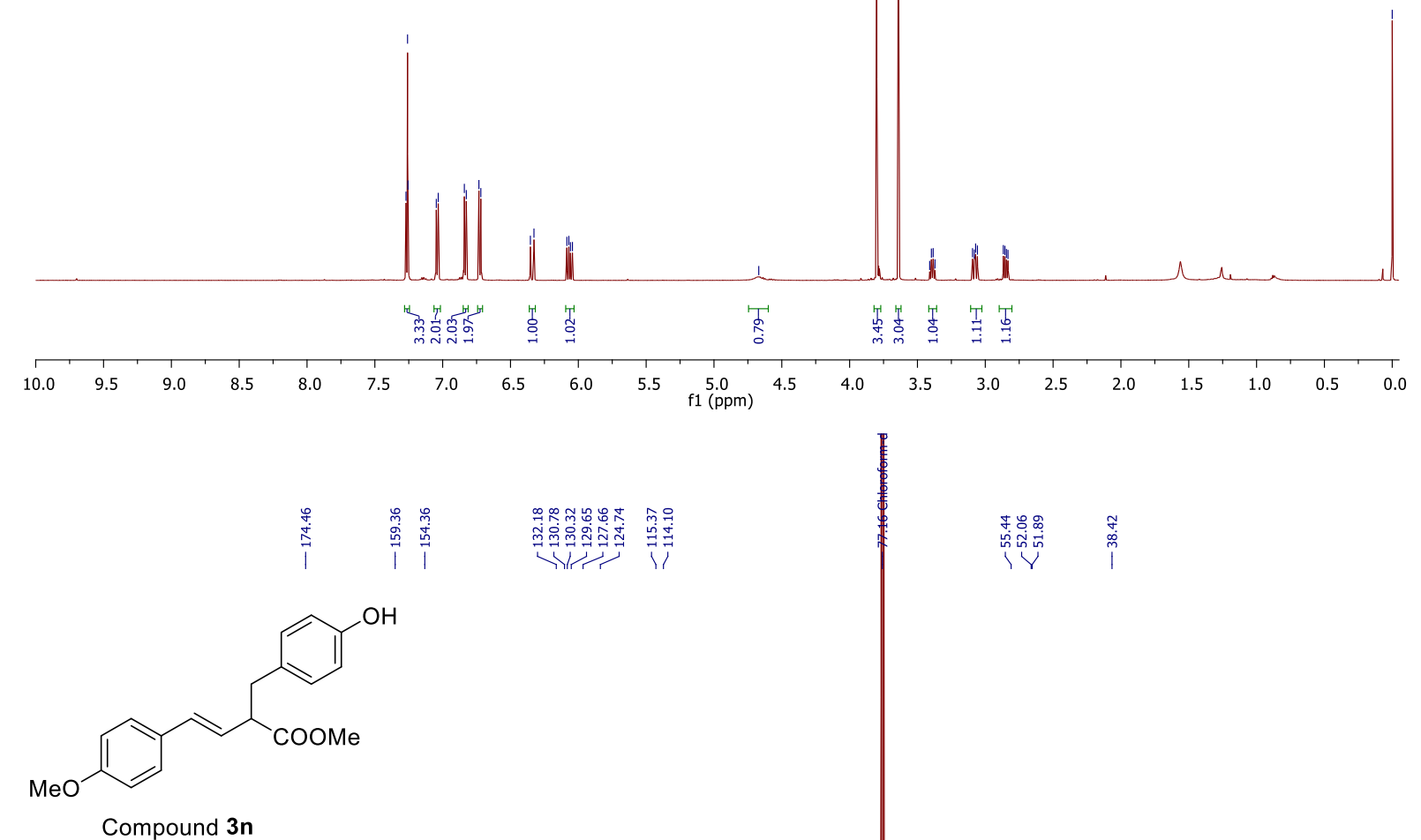

${ }^{13} \mathrm{C}-\mathrm{NMR}\left(151 \mathrm{MHz}, \mathrm{CDCl}_{3}\right)$

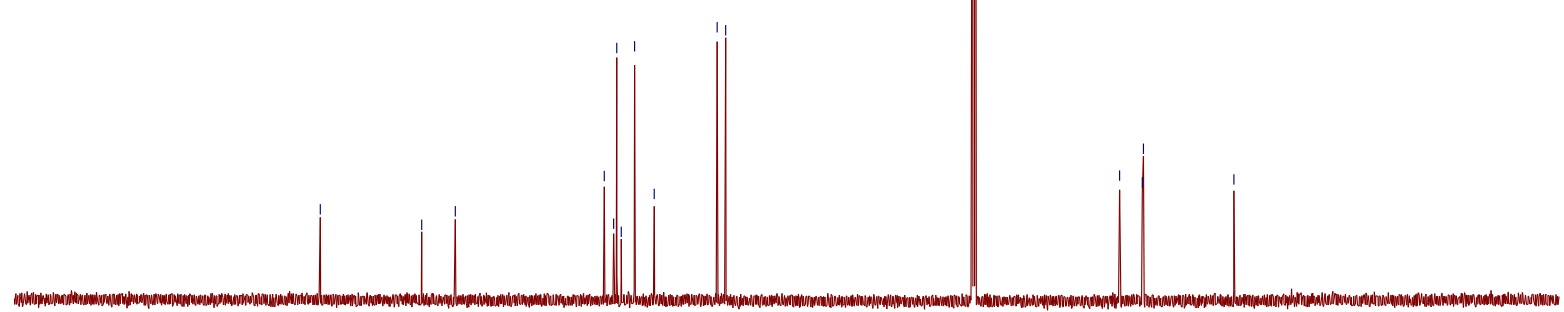

$\begin{array}{llllllllllllllllllllllllll}220 & 210 & 200 & 190 & 180 & 170 & 160 & 150 & 140 & 130 & 120 & 110 & 100 & 90 & 80 & 70 & 60 & 50 & 40 & 30 & 20 & 10 & 0 & -10\end{array}$ 


\subsection{5. ${ }^{1} \mathrm{H}$ and ${ }^{13} \mathrm{C}$ NMR spectra of $3 \mathrm{o}$}
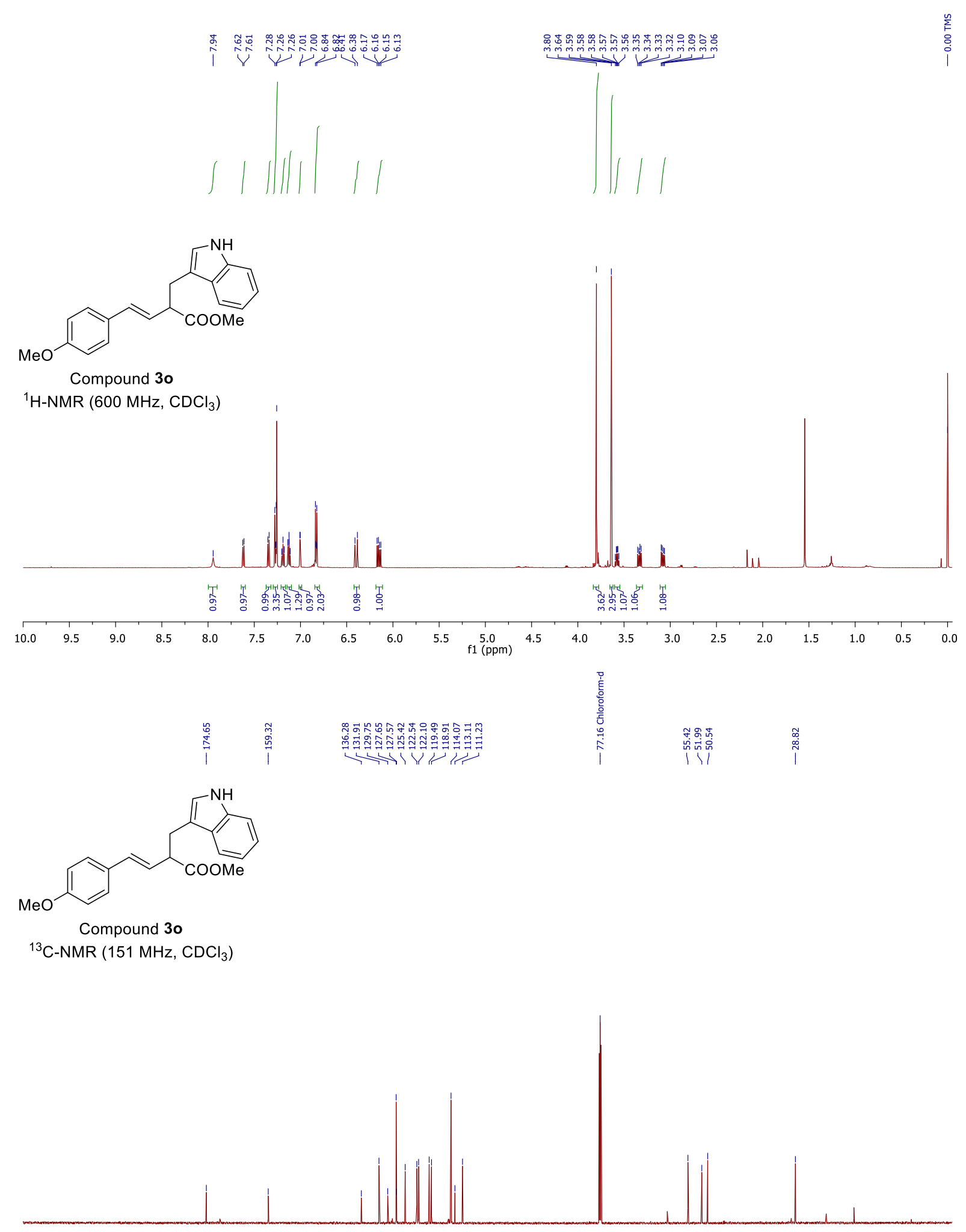

\begin{tabular}{rllllllllllllllllllllllll}
\hline 220 & 210 & 200 & 190 & 180 & 170 & 160 & 150 & 140 & 130 & 120 & 110 & 100 & 90 & 80 & 70 & 60 & 50 & 40 & 30 & 20 & 10 & 0 & -10
\end{tabular} 


\subsection{6. ${ }^{1} \mathrm{H}$ and ${ }^{13} \mathrm{C}$ NMR spectra of $3 p$}
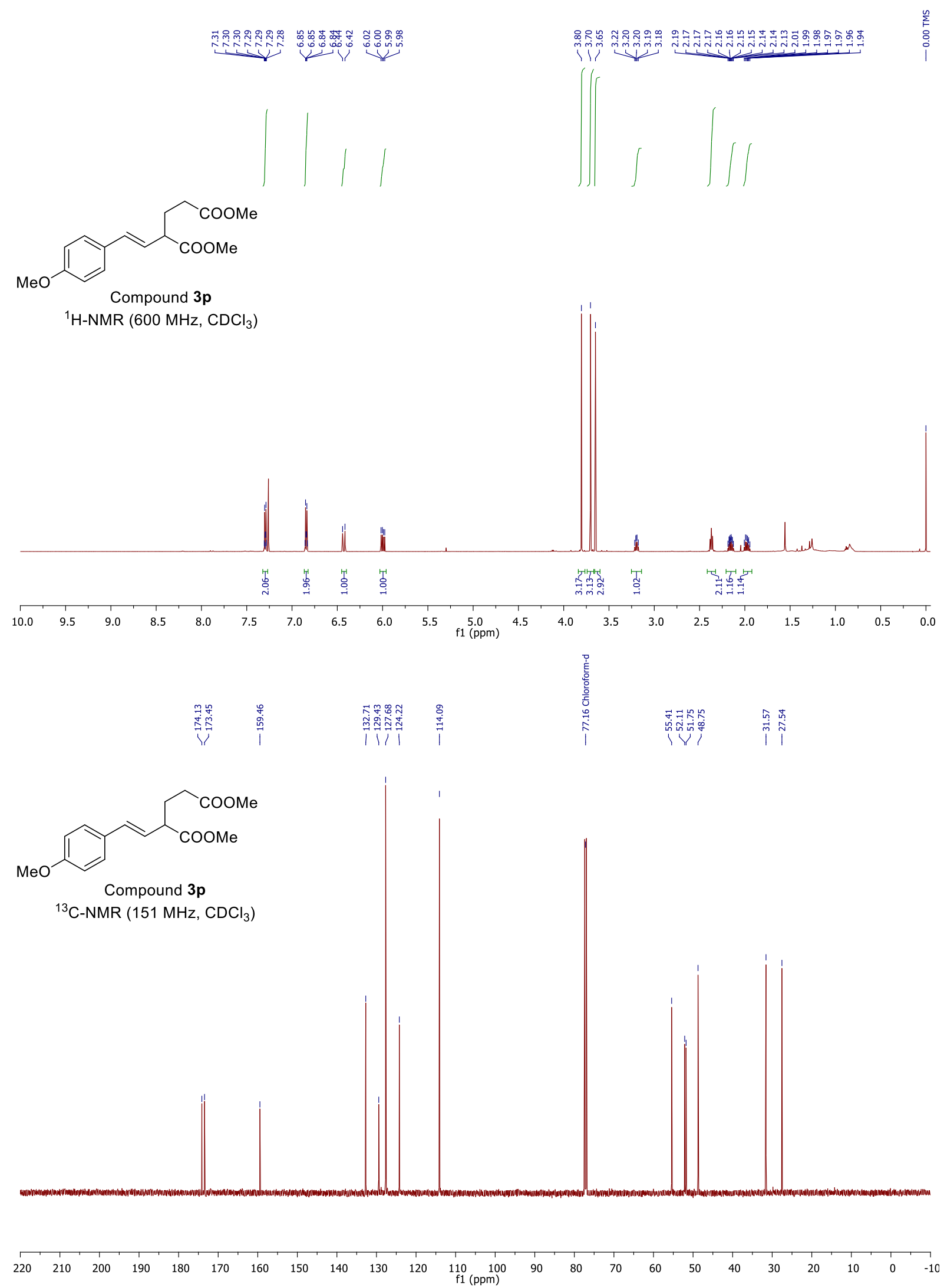


\subsection{7. ${ }^{1} \mathrm{H}$ and ${ }^{13} \mathrm{C}$ NMR spectra of $3 q$}
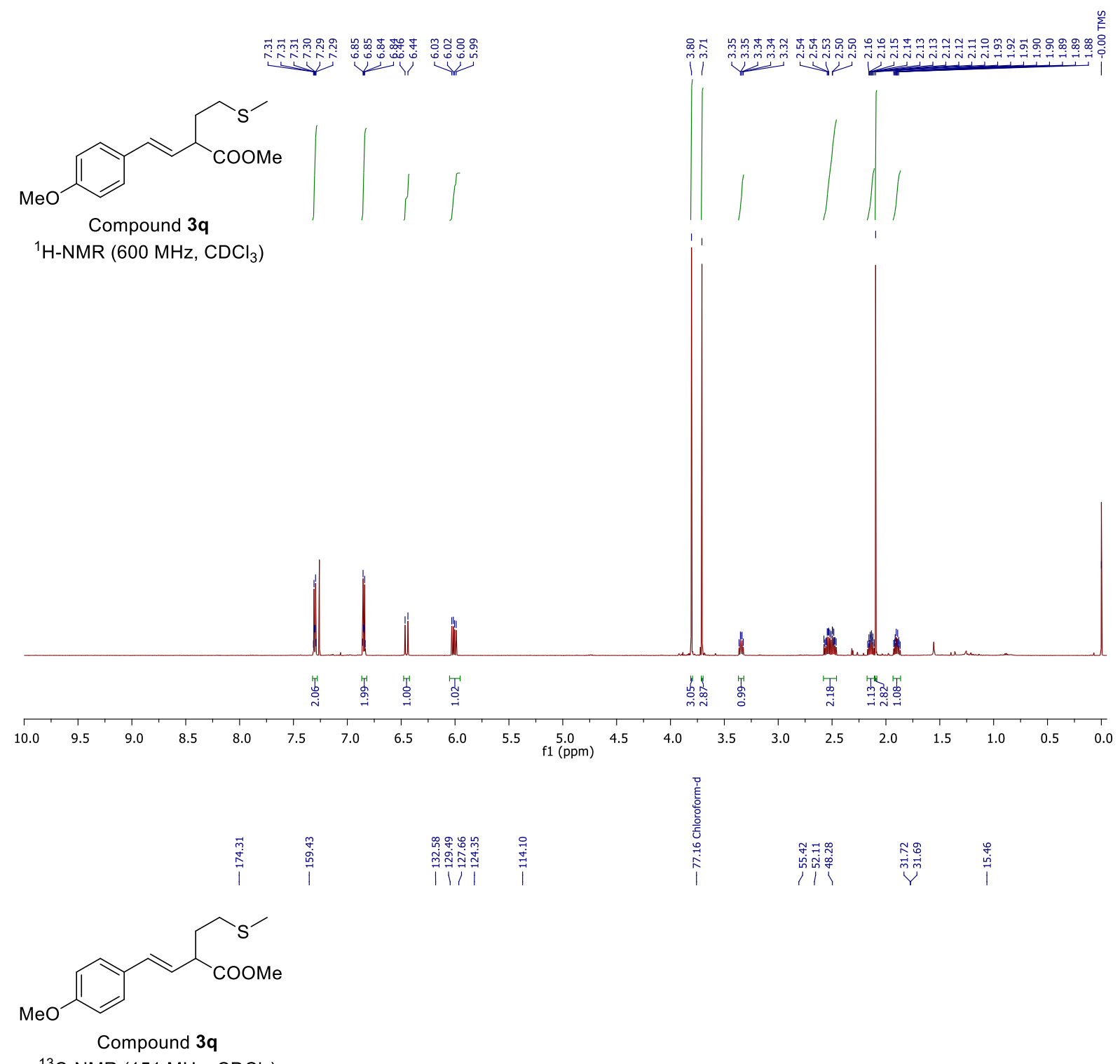

${ }^{13} \mathrm{C}-\mathrm{NMR}\left(151 \mathrm{MHz}, \mathrm{CDCl}_{3}\right)$

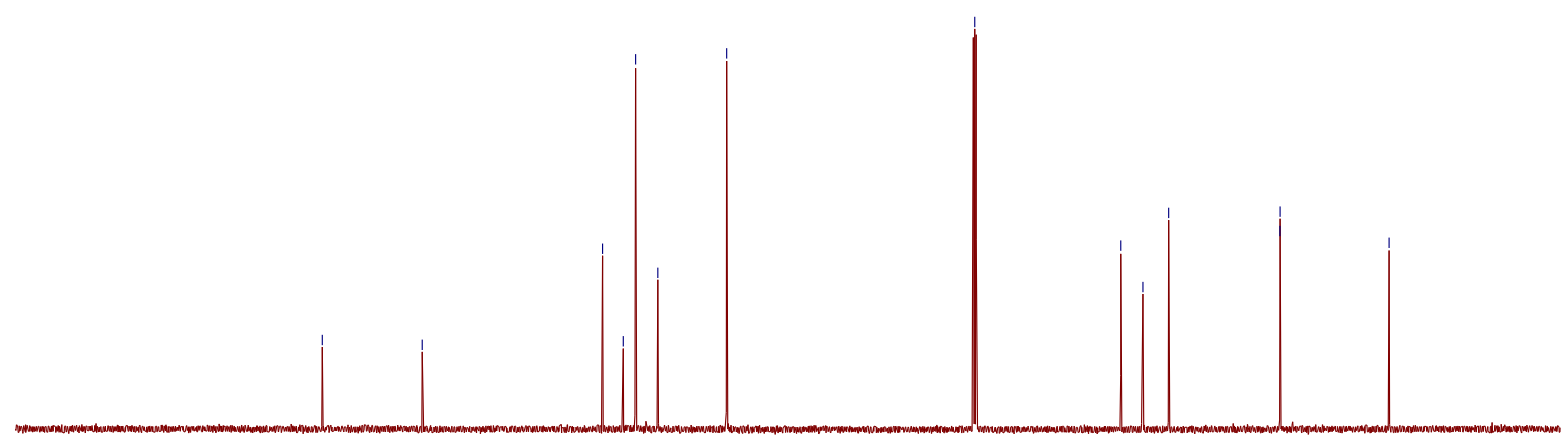

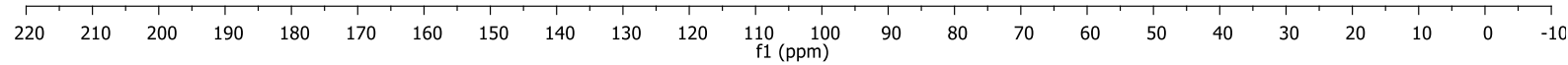




\subsection{8. ${ }^{1} \mathrm{H}$ and ${ }^{13} \mathrm{C}$ NMR spectra of $3 \mathrm{r}$}
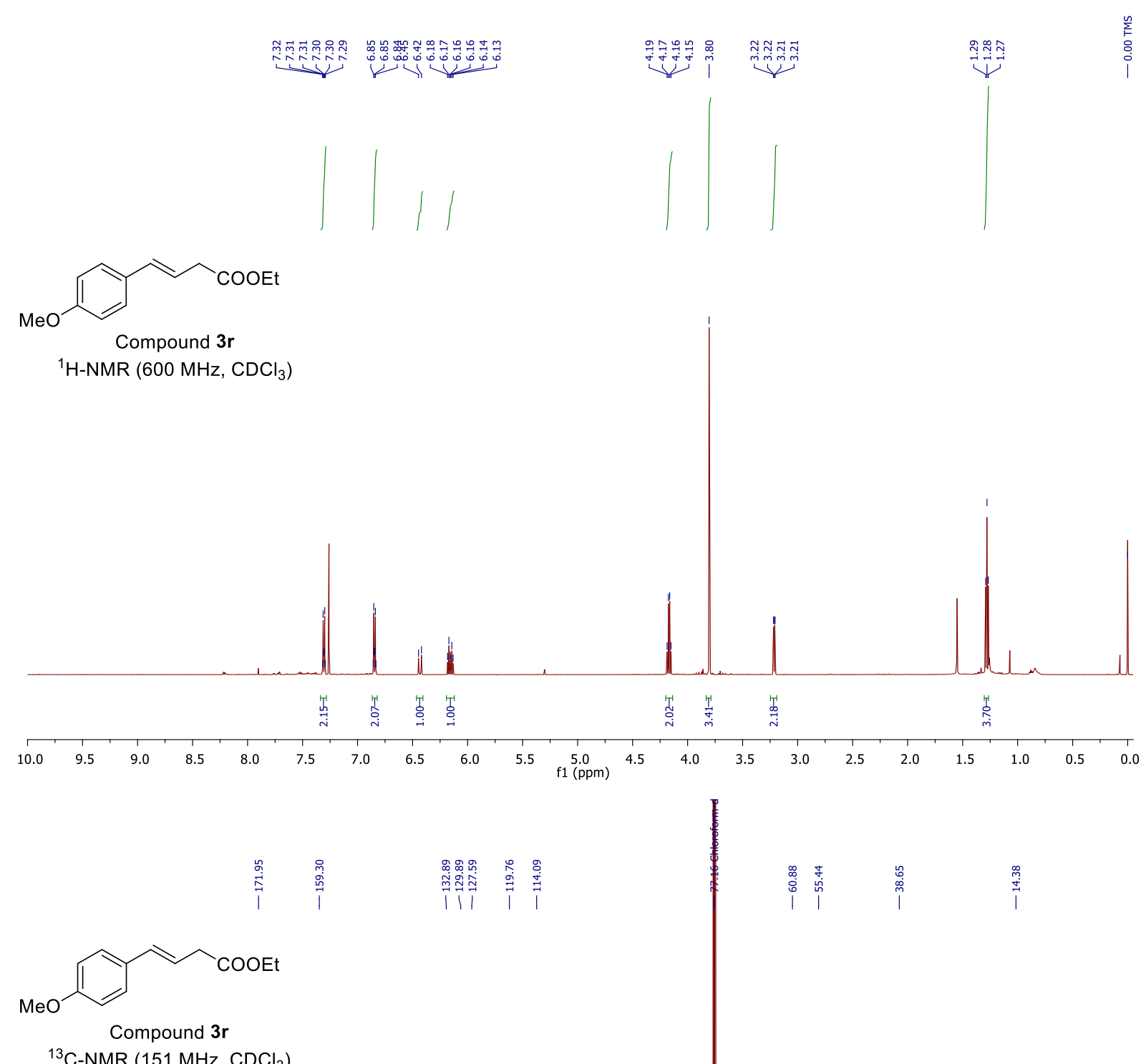

i

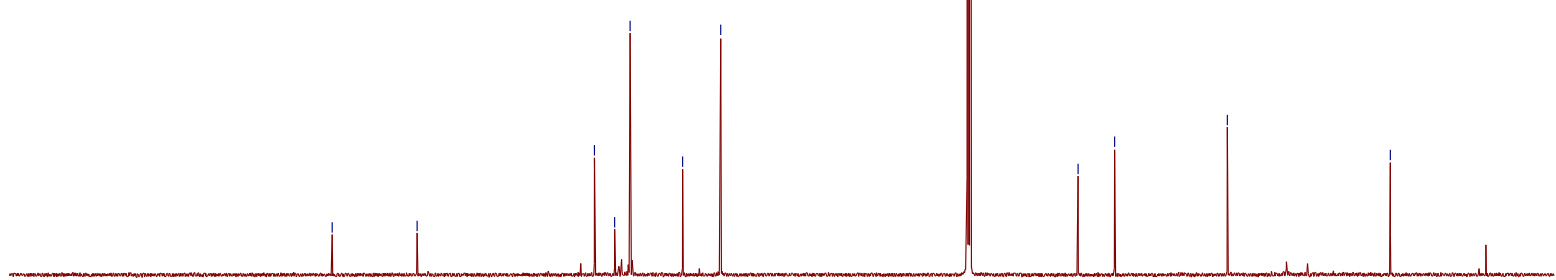

$\begin{array}{llllllllllllllllllllllllllll}220 & 210 & 200 & 190 & 180 & 170 & 160 & 150 & 140 & 130 & 120 & 110 & 100 & 90 & 80 & 70 & 60 & 50 & 40 & 30 & 20 & 10 & 0 & -10\end{array}$ 


\subsection{9. ${ }^{1} \mathrm{H}$ and ${ }^{13} \mathrm{C}$ NMR spectra of $3 \mathrm{~s}$}
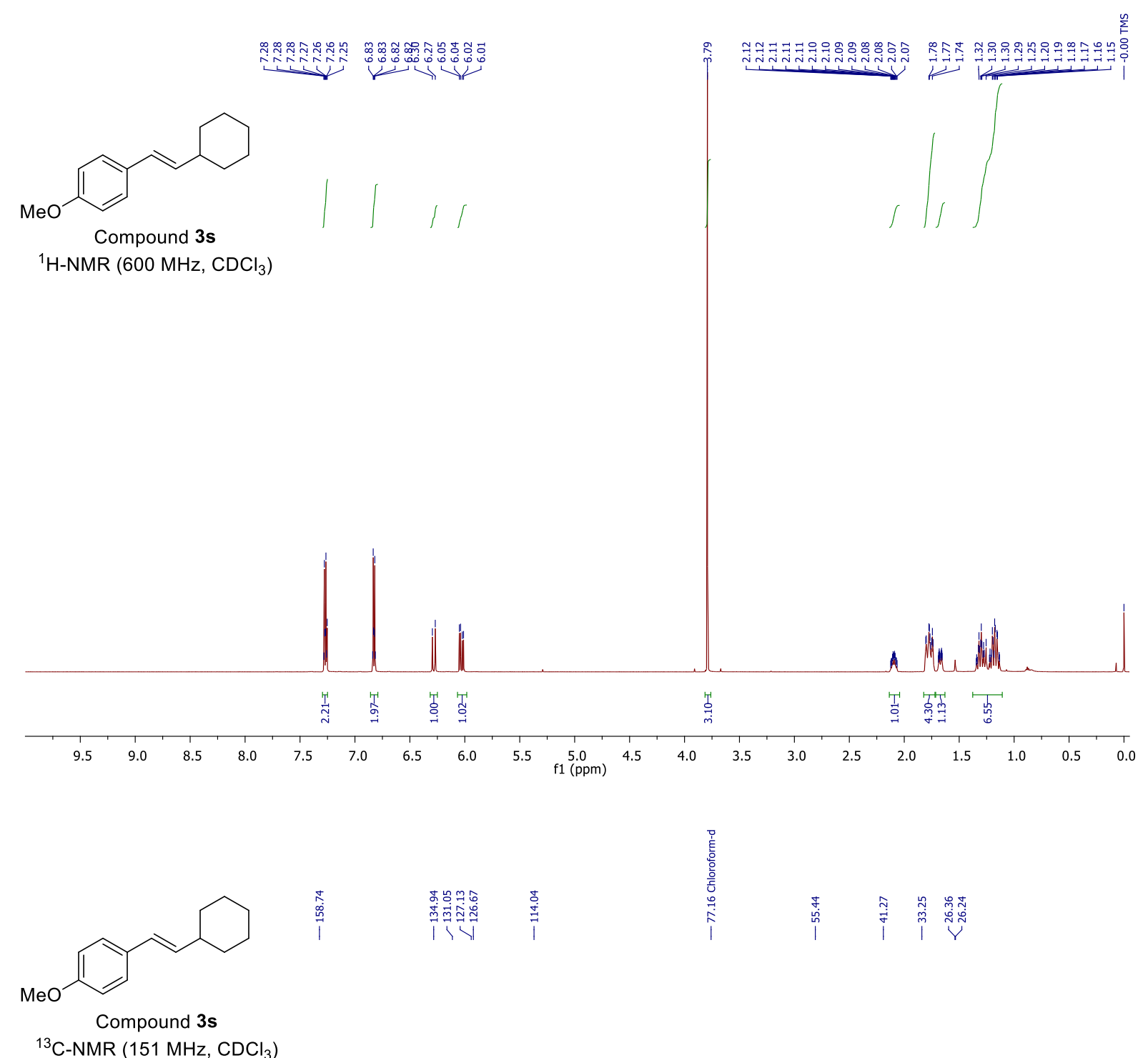

${ }^{13} \mathrm{C}-\mathrm{NMR}\left(151 \mathrm{MHz}, \mathrm{CDCl}_{3}\right)$

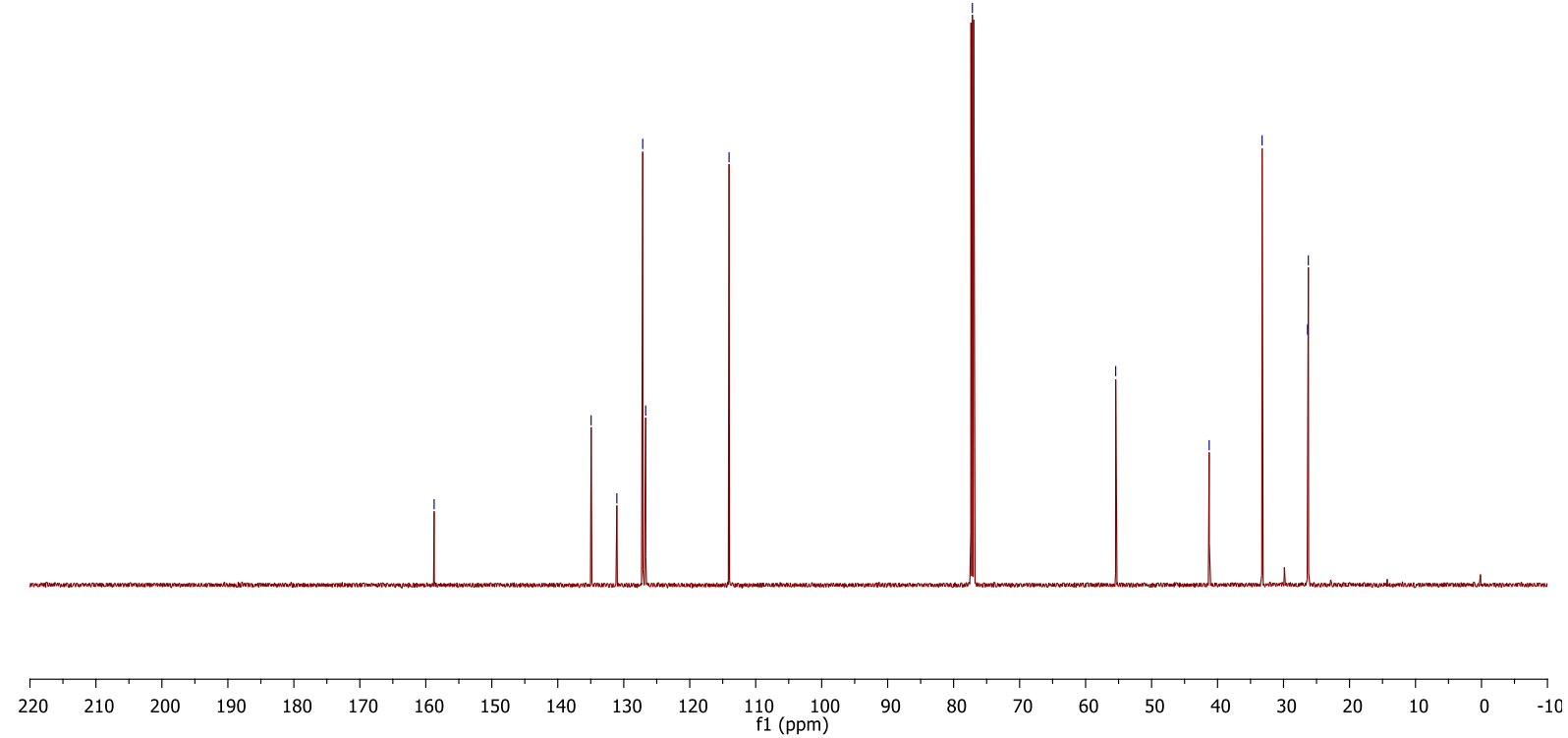




\subsection{0. ${ }^{1} \mathrm{H}$ and ${ }^{13} \mathrm{C}$ NMR spectra of $3 \mathrm{t}$}
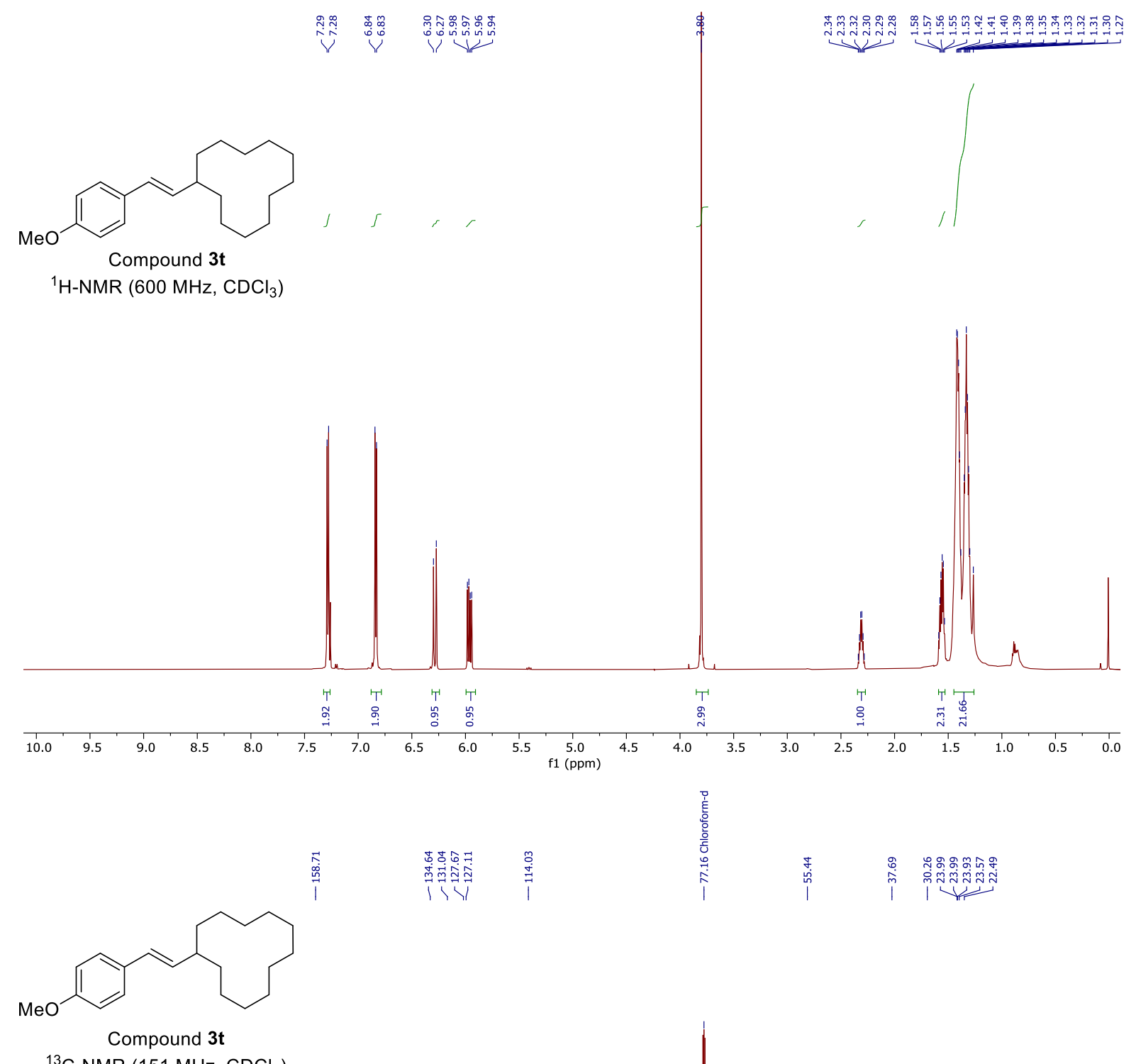

${ }^{13} \mathrm{C}-\mathrm{NMR}\left(151 \mathrm{MHz}, \mathrm{CDCl}_{3}\right)$

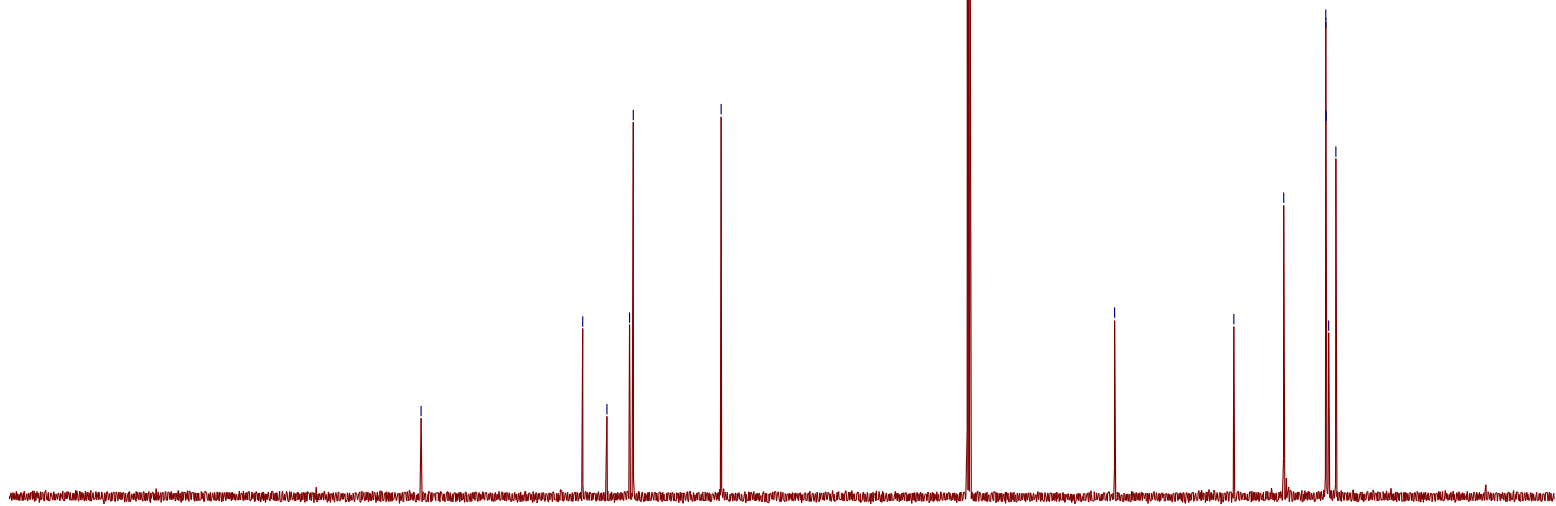

$\begin{array}{llllllllllllllllllllllllll}220 & 210 & 200 & 190 & 180 & 170 & 160 & 150 & 140 & 130 & 120 & \underset{f 1}{110} \underset{(\mathrm{ppm})}{100} & 90 & 80 & 70 & 60 & 50 & 40 & 30 & 20 & 10 & 0 & -10\end{array}$ 


\subsection{1. ${ }^{1} \mathrm{H}$ and ${ }^{13} \mathrm{C}$ NMR spectra of $3 \mathrm{u}$}
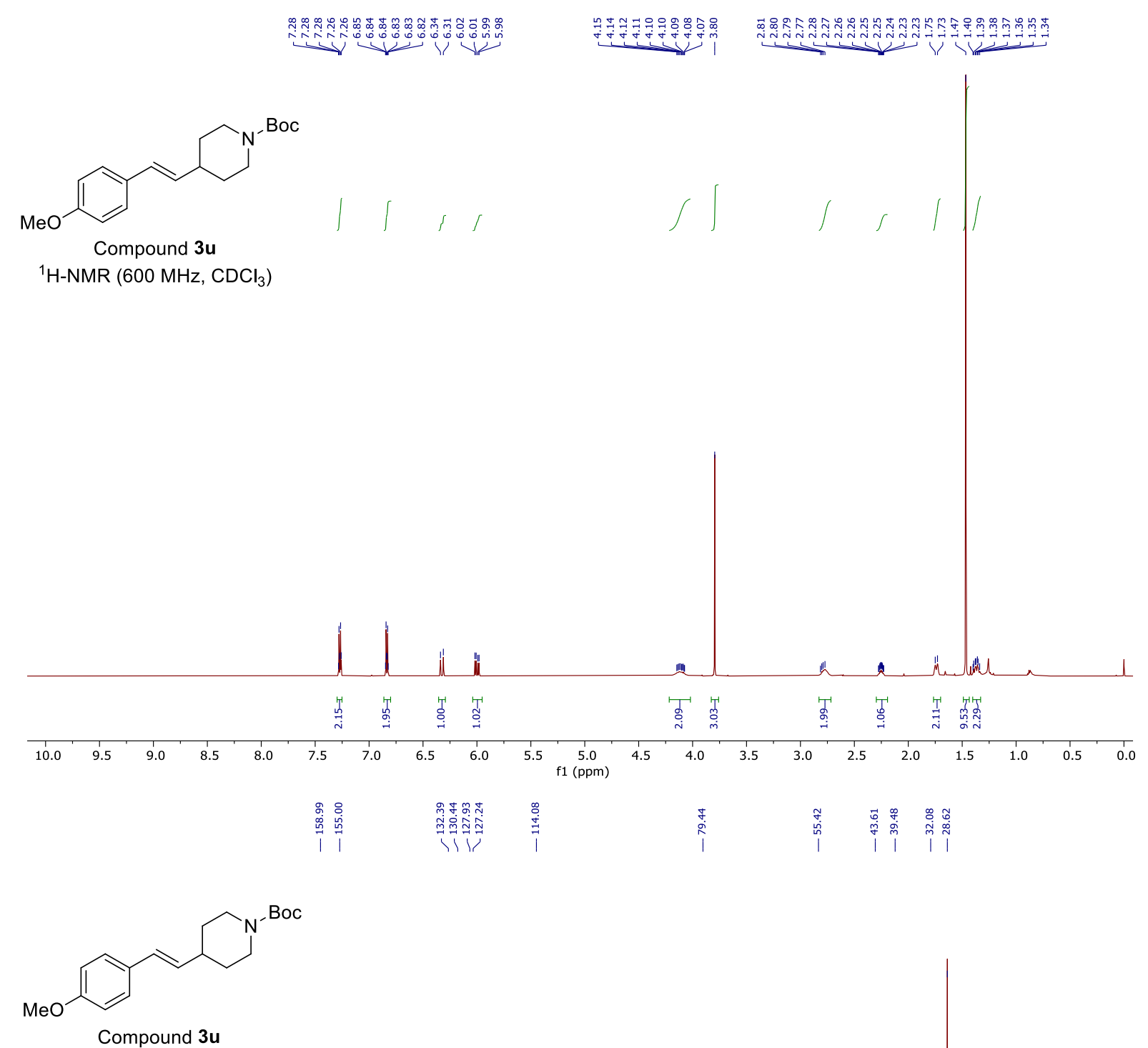

${ }^{13} \mathrm{C}-\mathrm{NMR}\left(151 \mathrm{MHz}, \mathrm{CDCl}_{3}\right)$

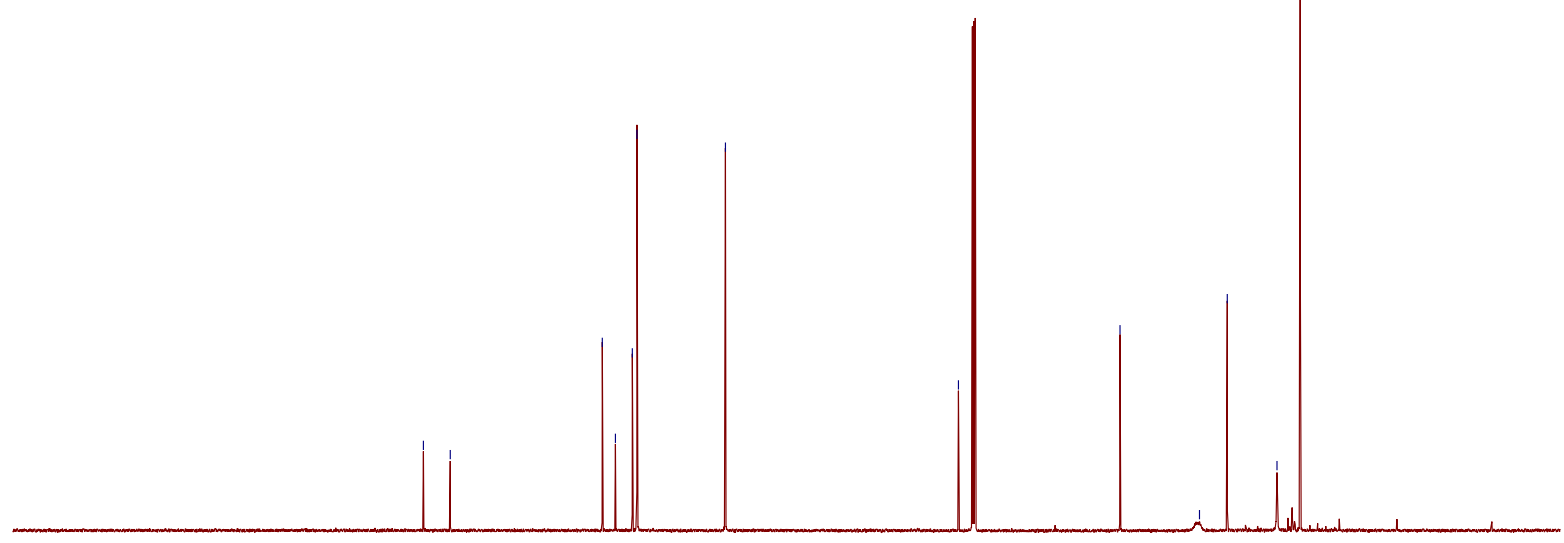

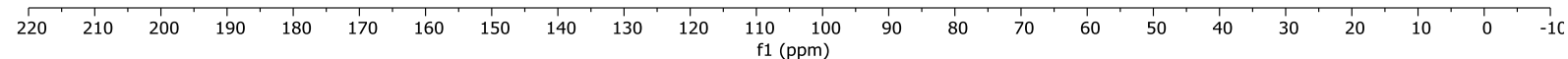




\subsection{2. ${ }^{1} \mathrm{H}$ and ${ }^{13} \mathrm{C}$ NMR spectra of $3 \mathrm{v}$}
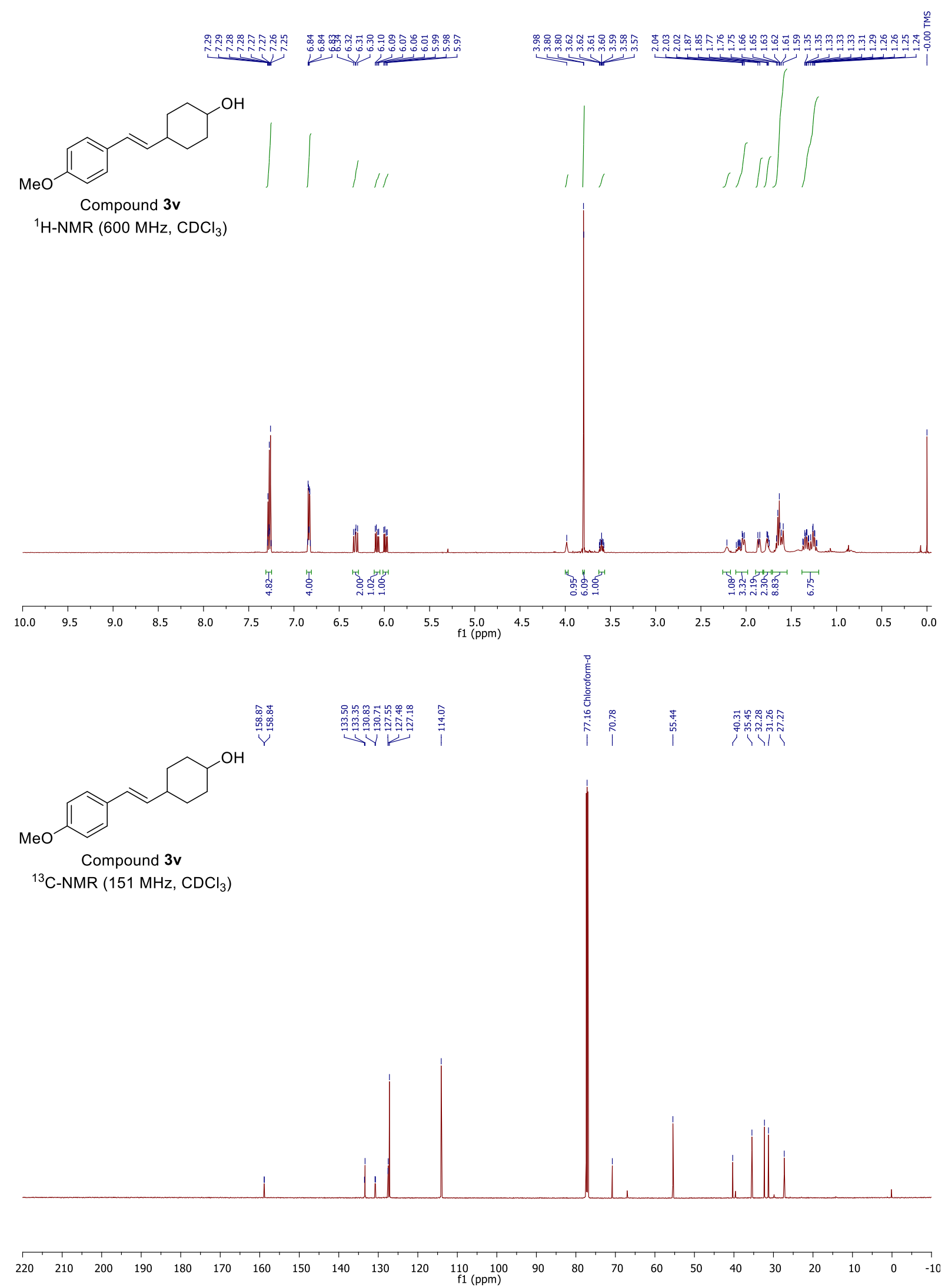

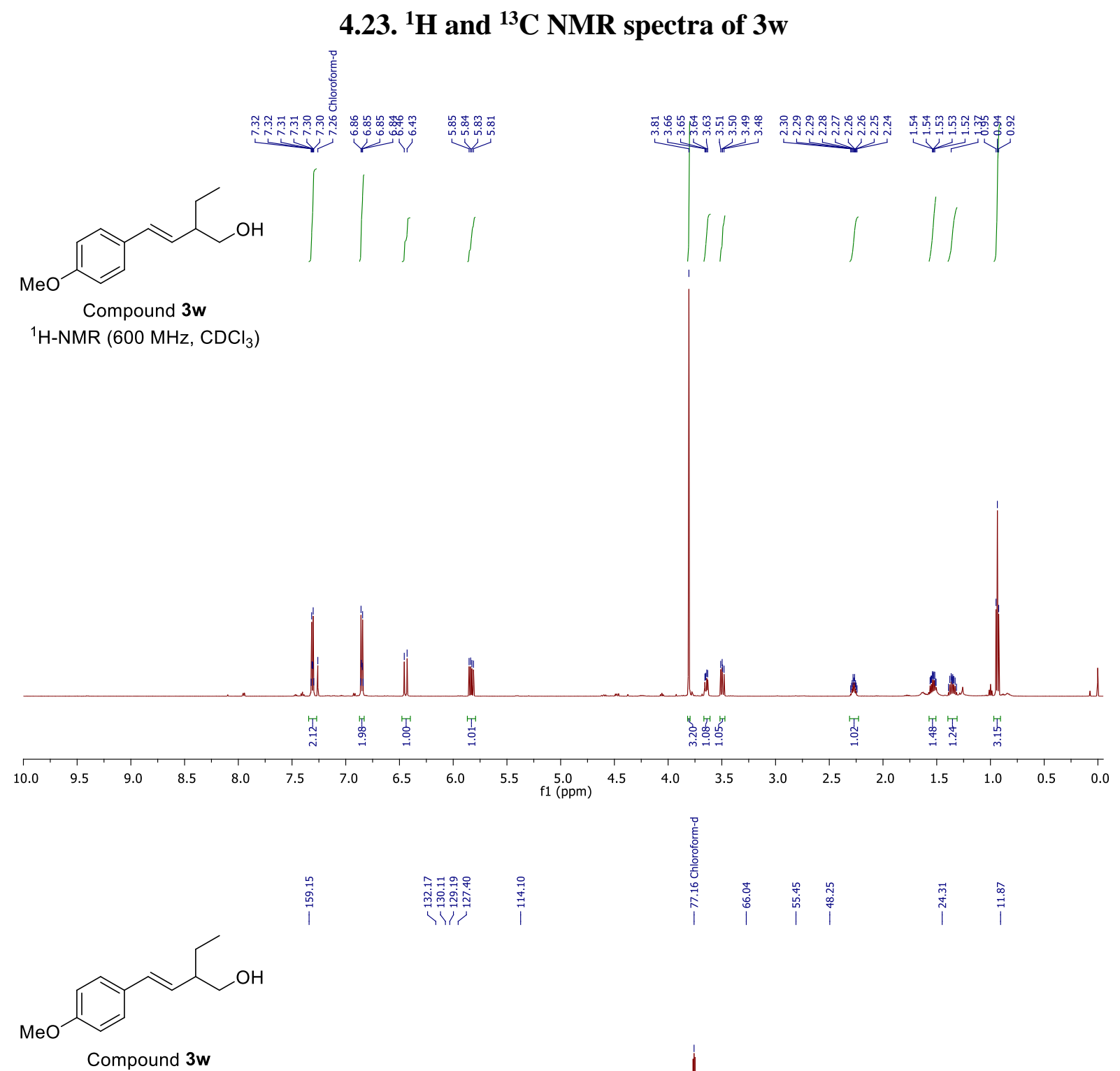

${ }^{13} \mathrm{C}-\mathrm{NMR}\left(151 \mathrm{MHz}, \mathrm{CDCl}_{3}\right)$

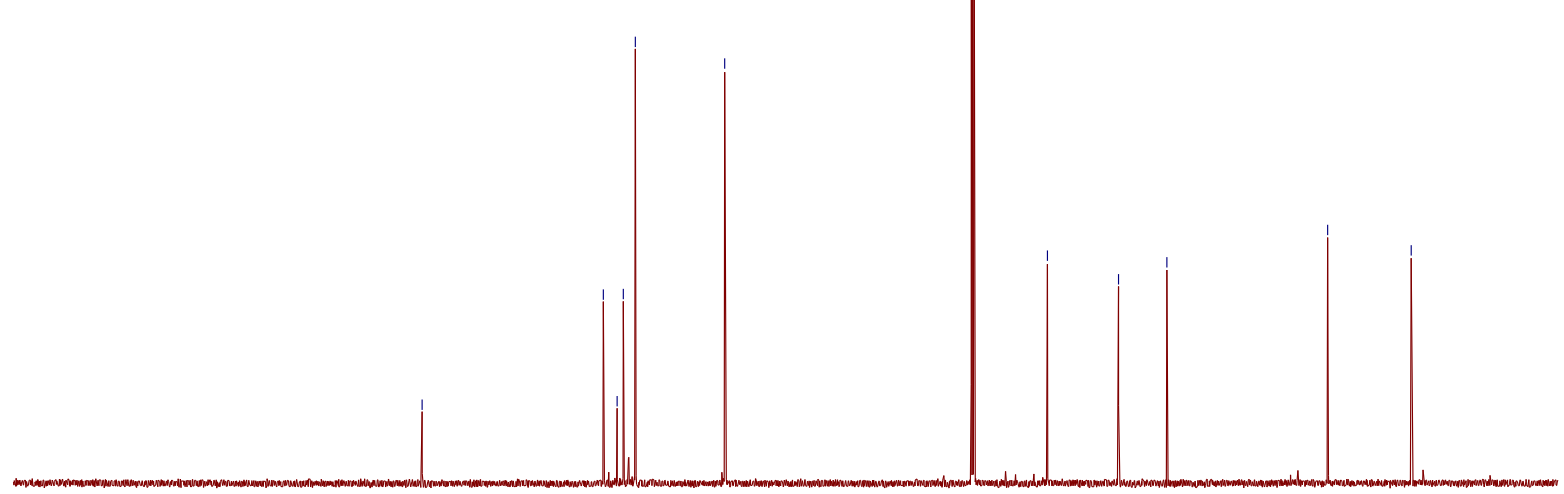

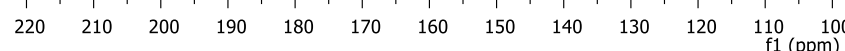




\subsection{4. ${ }^{1} \mathrm{H}$ and ${ }^{13} \mathrm{C}$ NMR spectra of $3 y$}
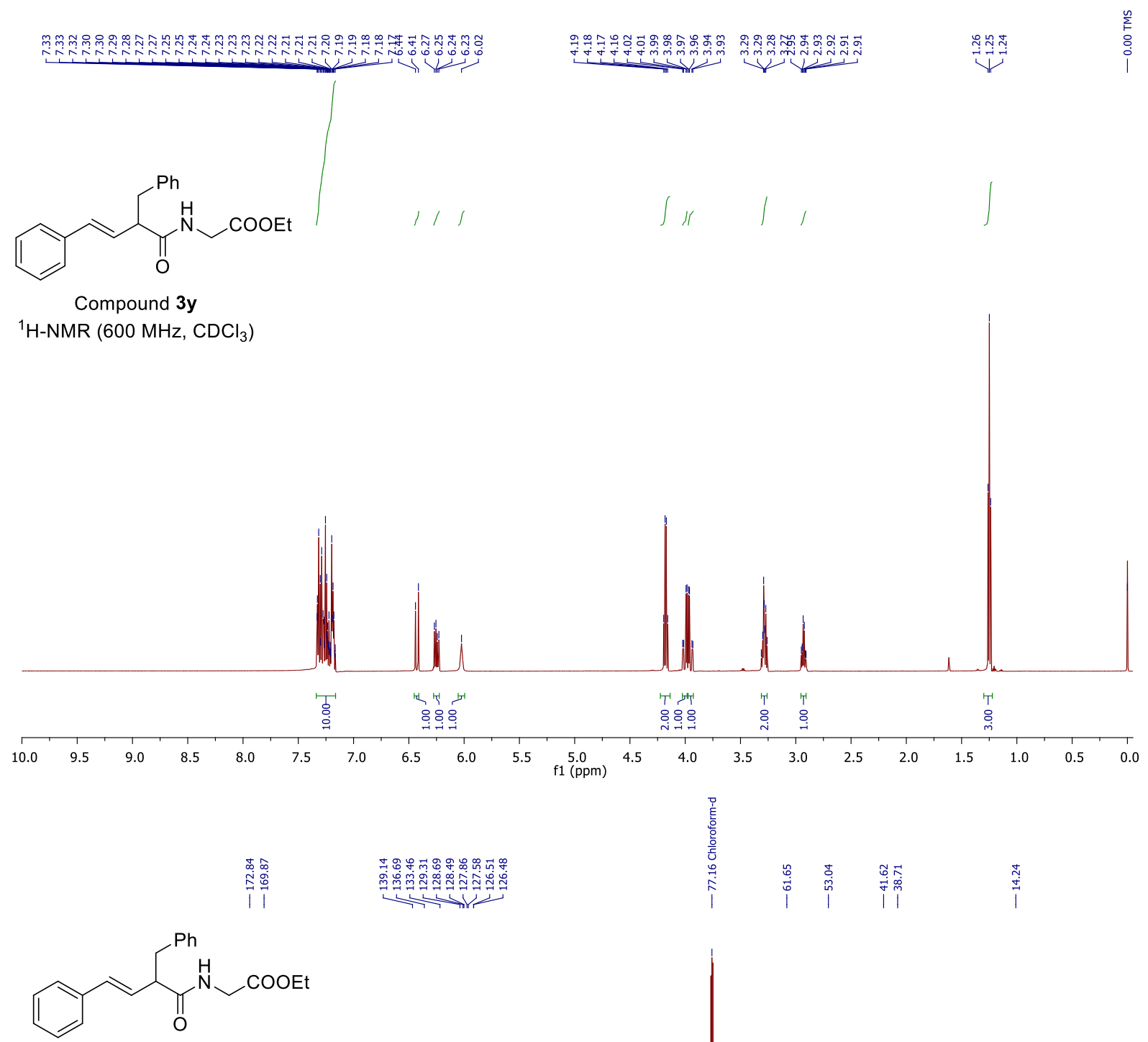

Compound $3 y$

${ }^{13} \mathrm{C}-\mathrm{NMR}\left(151 \mathrm{MHz}, \mathrm{CDCl}_{3}\right)$

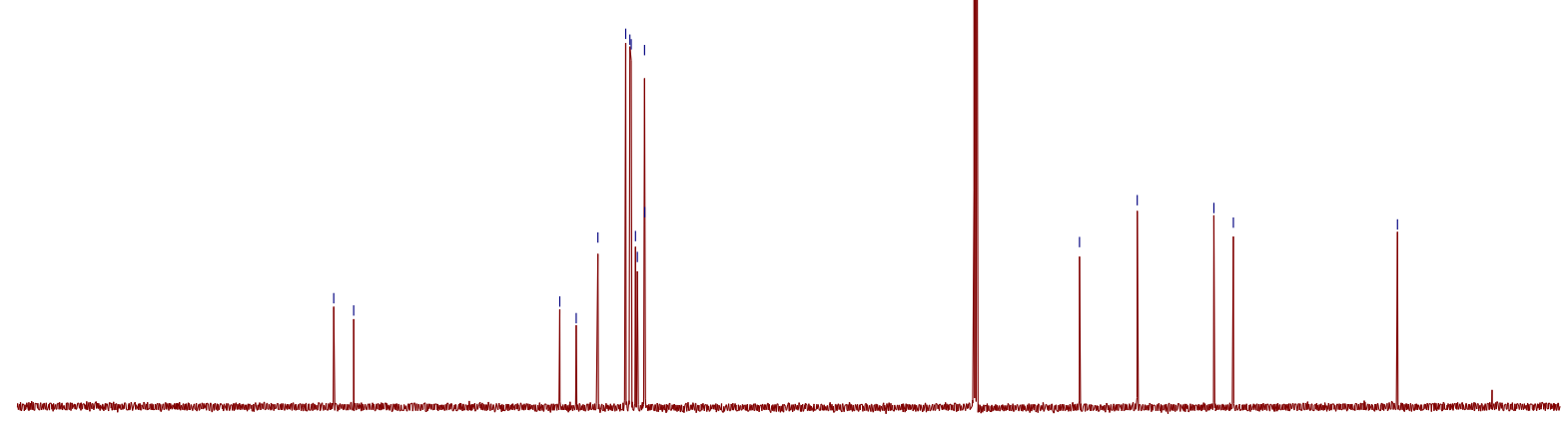

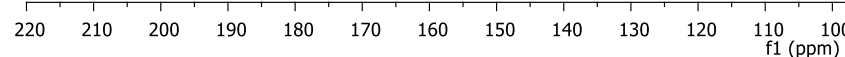

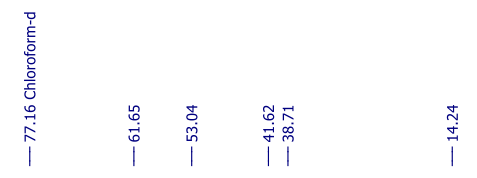




\subsection{5. ${ }^{1} \mathrm{H}$ and ${ }^{13} \mathrm{C}$ NMR spectra of $3 \mathrm{z}$}
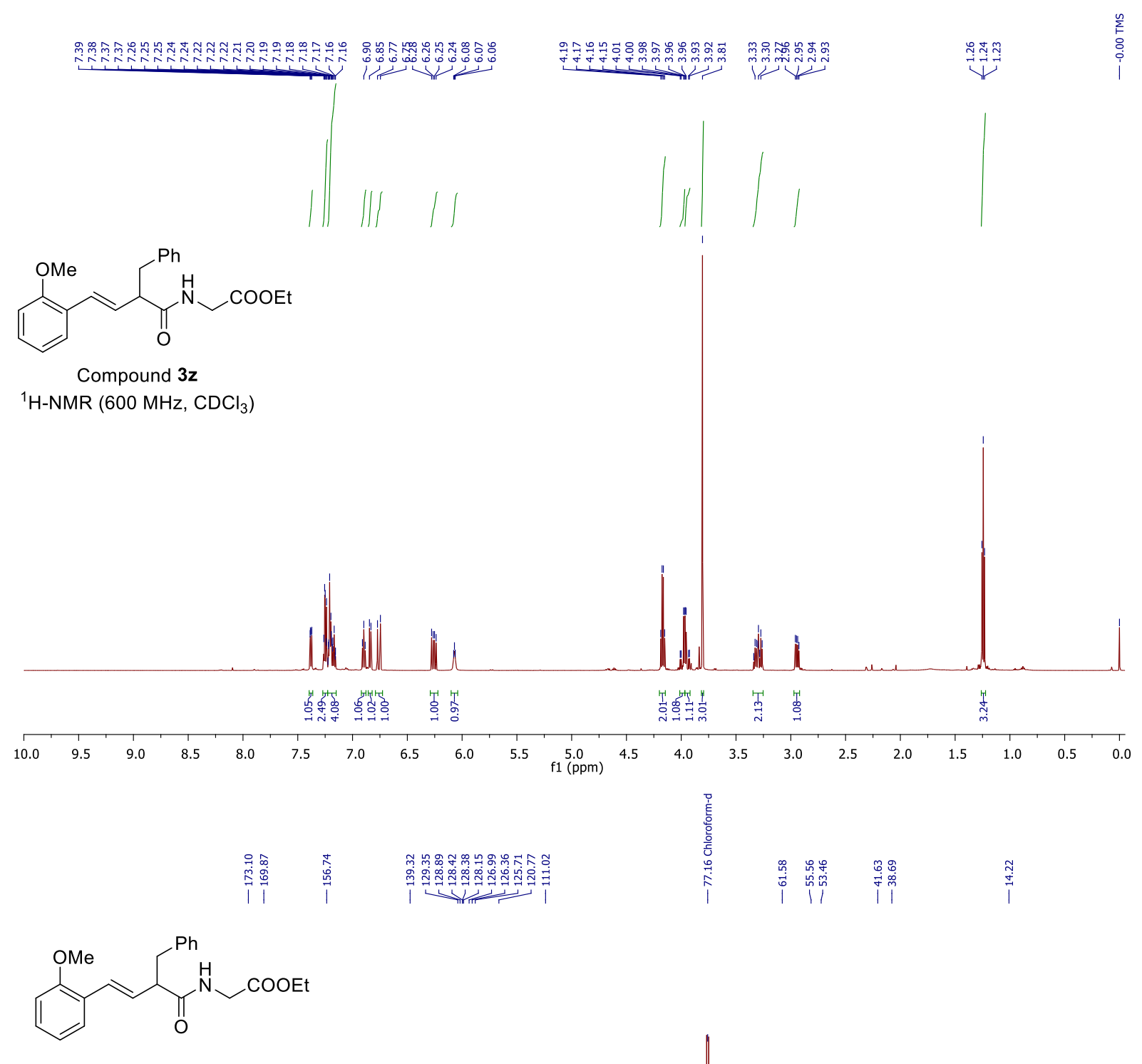

Compound $\mathbf{3 z}$

${ }^{13} \mathrm{C}-\mathrm{NMR}\left(151 \mathrm{MHz}, \mathrm{CDCl}_{3}\right)$

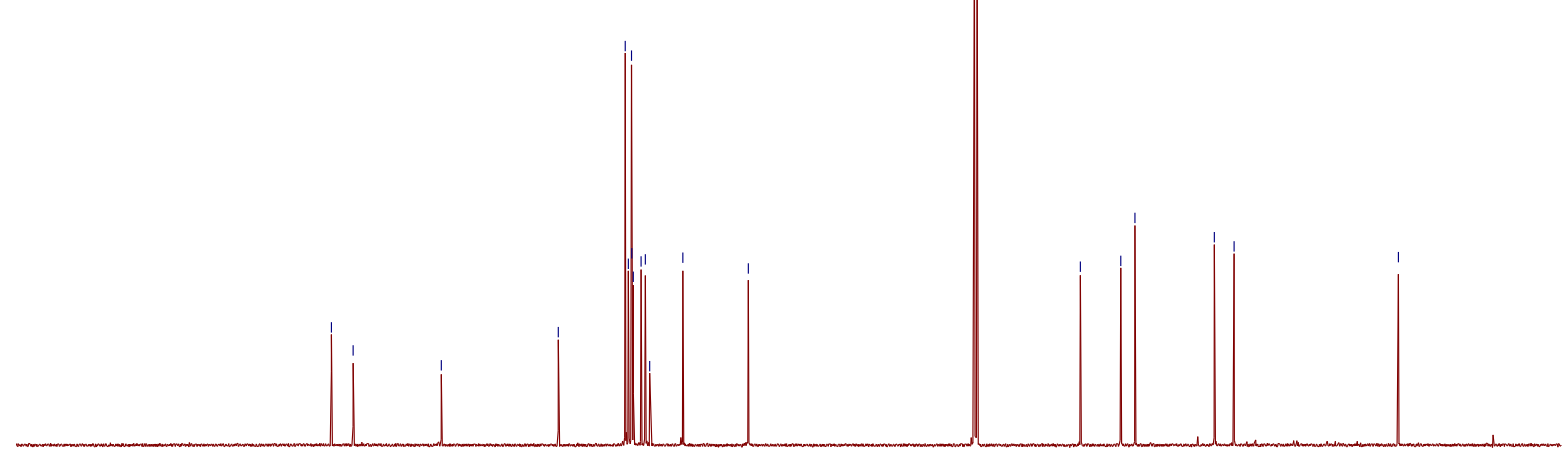

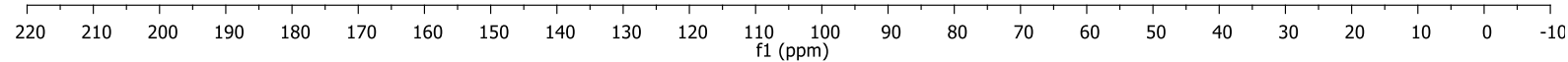




\subsection{6. ${ }^{1} \mathrm{H}$ and ${ }^{13} \mathrm{C}$ NMR spectra of 3aa}
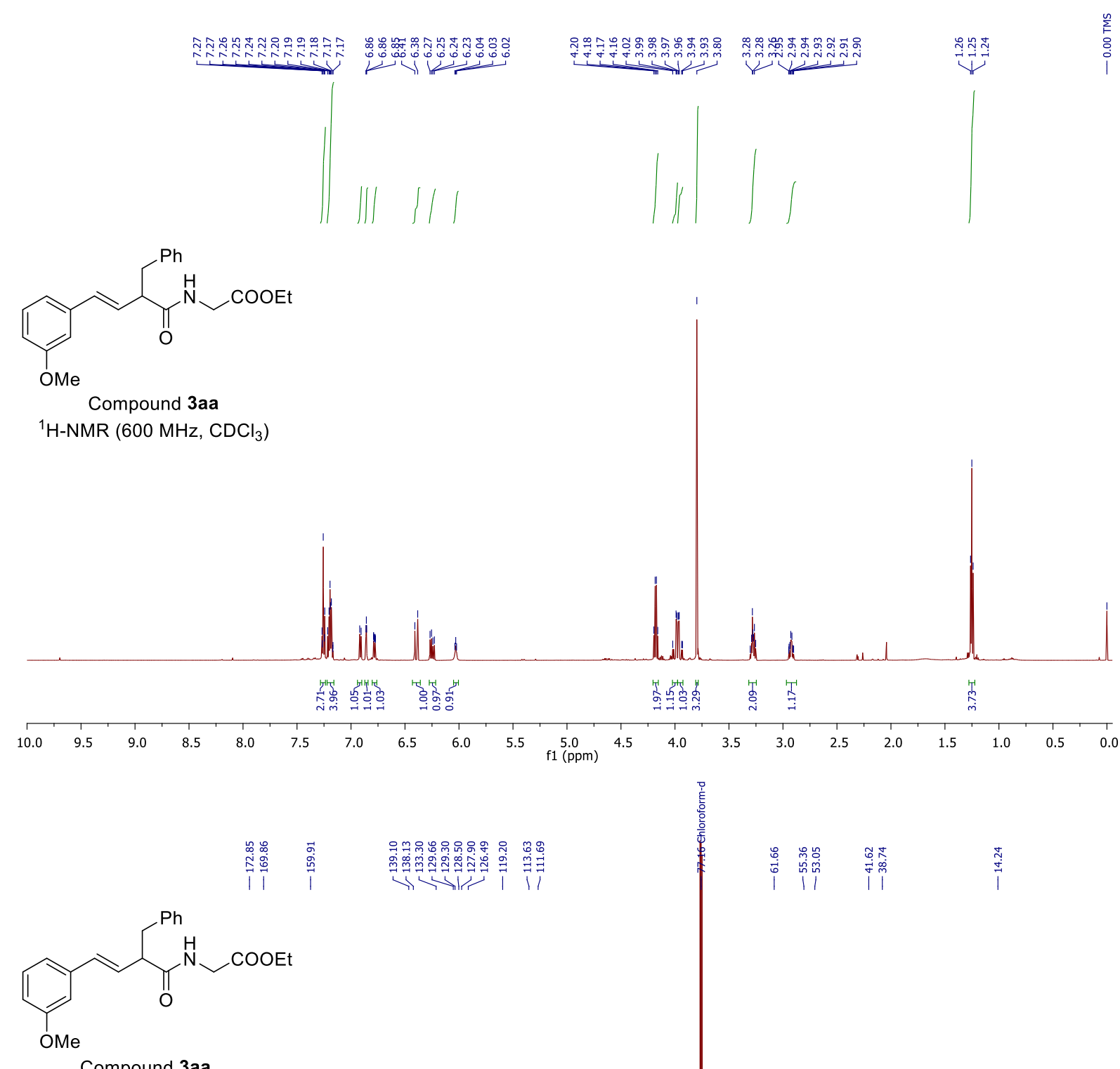

Compound 3aa

${ }^{13} \mathrm{C}-\mathrm{NMR}\left(151 \mathrm{MHz}, \mathrm{CDCl}_{3}\right)$

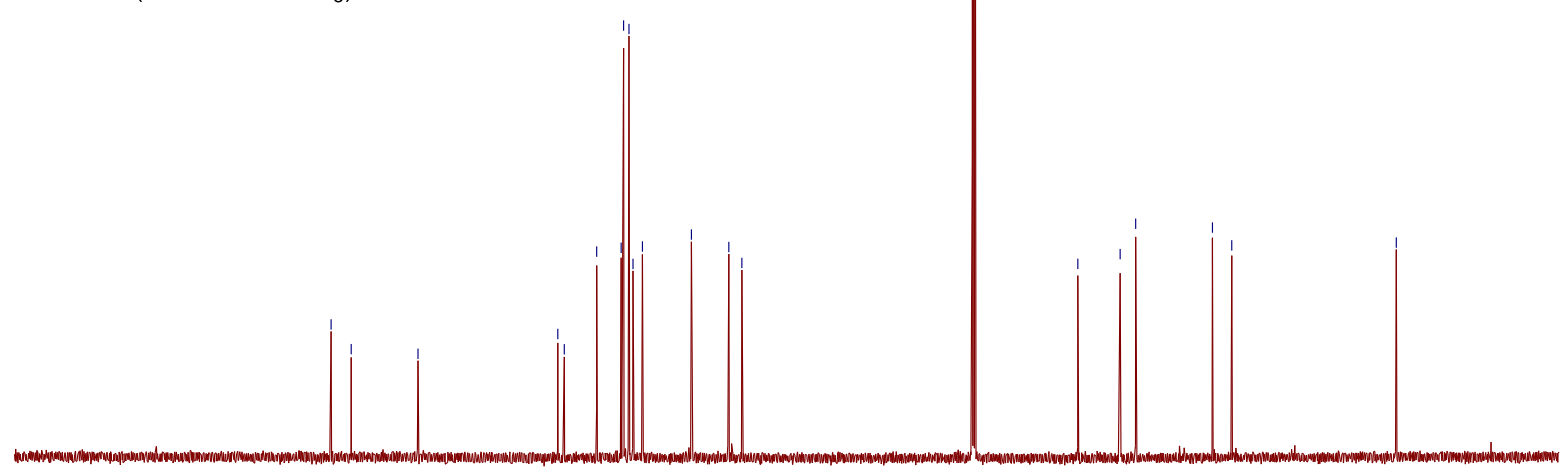

\begin{tabular}{rlllllllllllllllllllllllllll}
\hline 220 & 210 & 200 & 190 & 180 & 170 & 160 & 150 & 140 & 130 & 120 & 110 & 100 & 90 & 80 & 70 & 60 & 50 & 40 & 30 & 20 & 10 & 0 & -10
\end{tabular} 


\subsection{7. ${ }^{1} \mathrm{H}$ and ${ }^{13} \mathrm{C}$ NMR spectra of $3 \mathrm{ab}$}
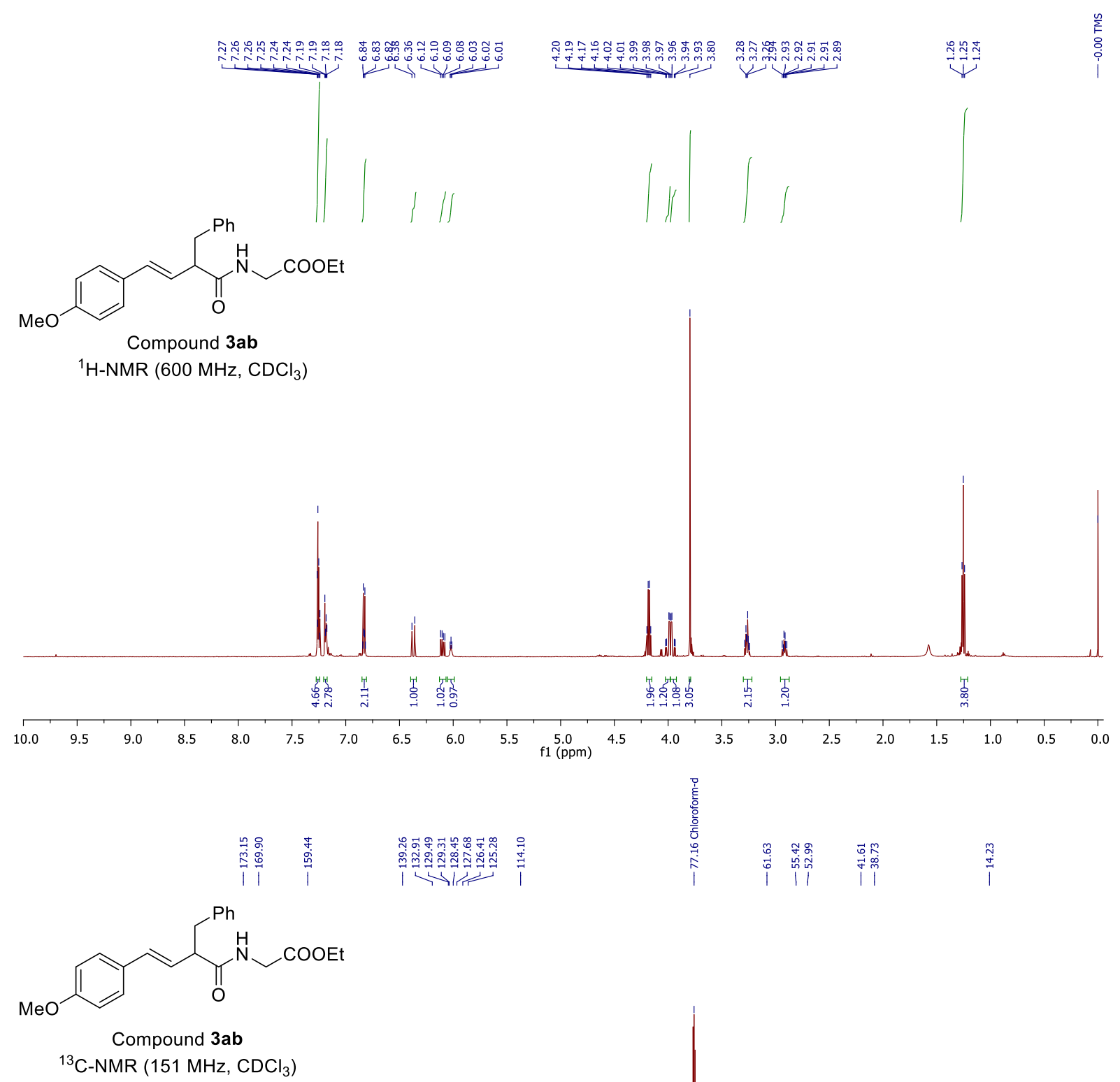

|

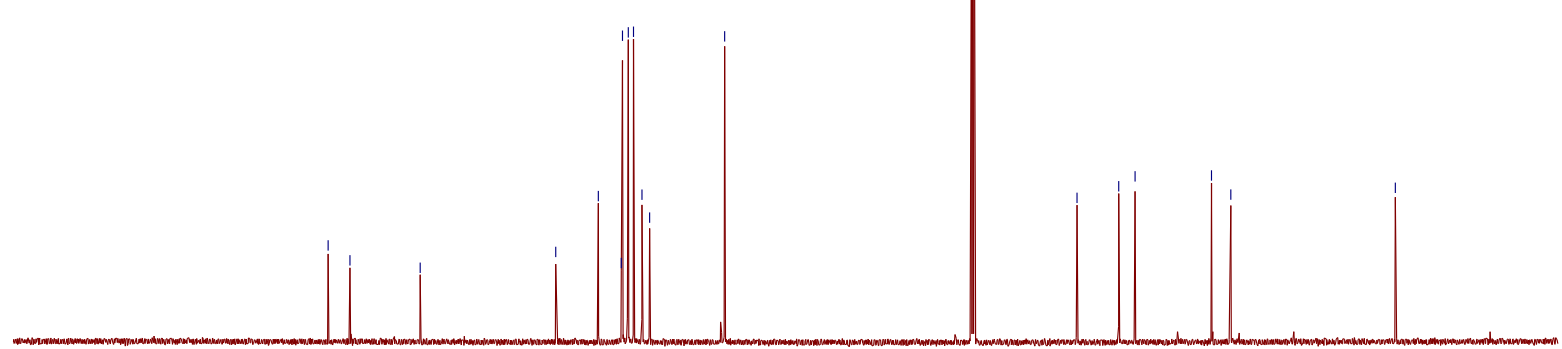

$\begin{array}{llllllllllllllllllllllllllllllll}220 & 210 & 200 & 190 & 180 & 170 & 160 & 150 & 140 & 130 & 120 & 110 & 100 & 90 & 80 & 70 & 60 & 50 & 40 & 30 & 20 & 10 & 0 & -10\end{array}$ 


\subsection{8. ${ }^{1} \mathrm{H}$ and ${ }^{13} \mathrm{C}$ NMR spectra of $3 a c$}
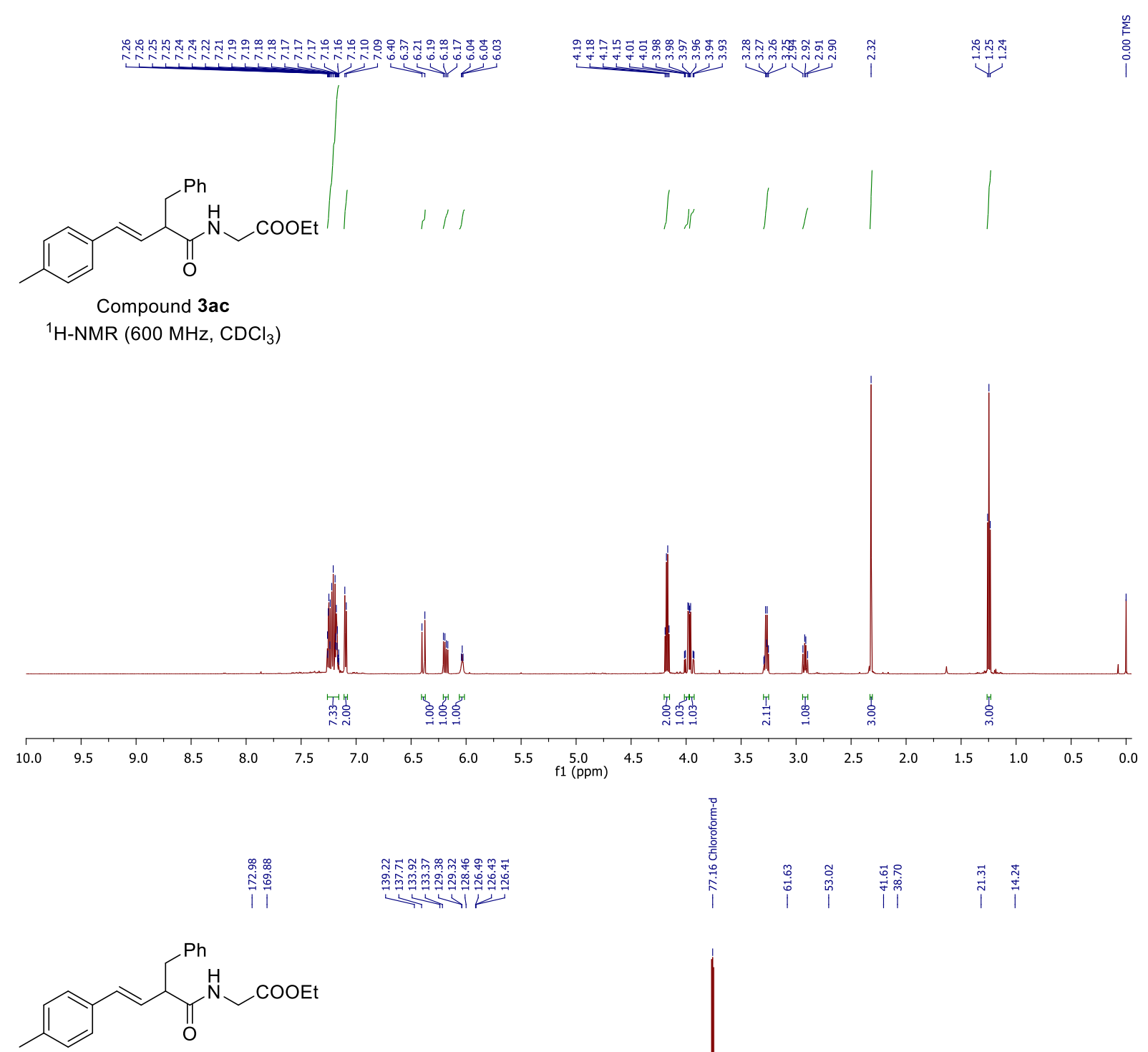

Compound 3ac

${ }^{13} \mathrm{C}-\mathrm{NMR}\left(151 \mathrm{MHz}, \mathrm{CDCl}_{3}\right)$

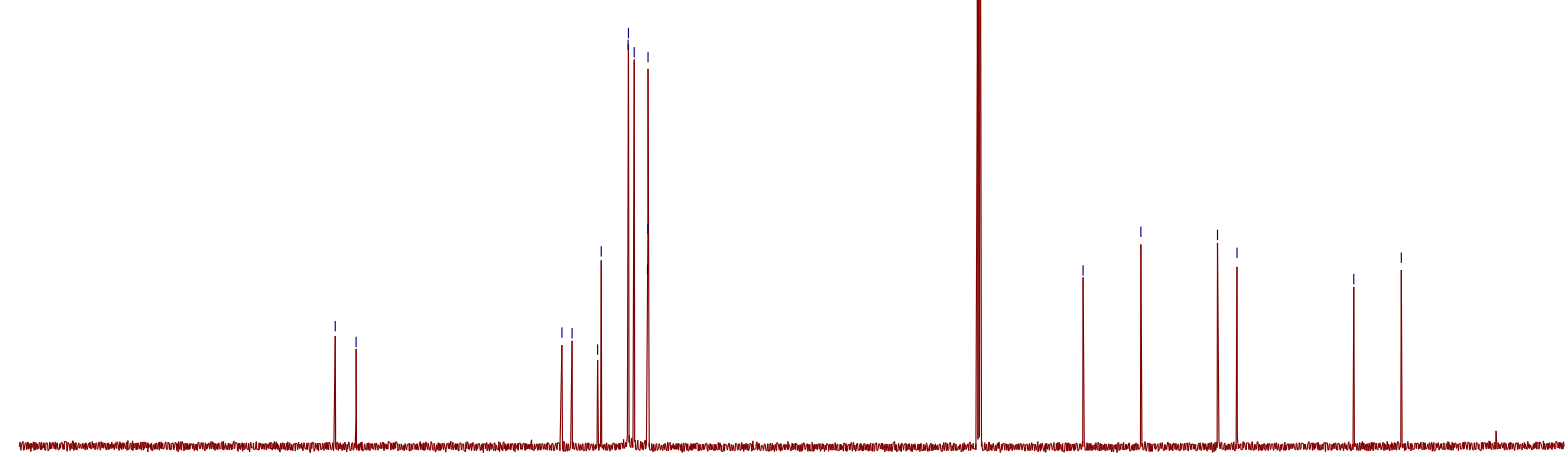

$\begin{array}{rlllllllllllllllllllllll}220 & 210 & 200 & 190 & 180 & 170 & 160 & 150 & 140 & 130 & 120 & \begin{array}{c}110 \\ \mathrm{f} 1(\mathrm{ppm})\end{array} & 100 & 80 & 70 & 60 & 50 & 40 & 30 & 20 & 10 & 0 & -10\end{array}$ 


\subsection{9. ${ }^{1} \mathrm{H}$ and ${ }^{13} \mathrm{C}$ NMR spectra of $3 \mathrm{ad}$}

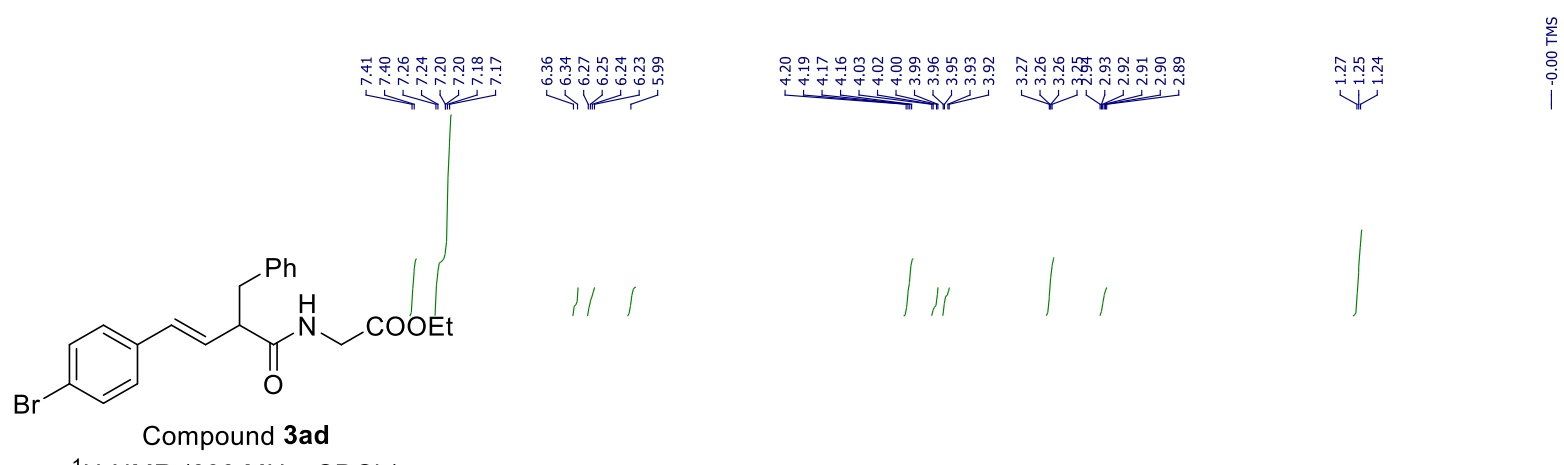

${ }^{1} \mathrm{H}-\mathrm{NMR}\left(600 \mathrm{MHz}, \mathrm{CDCl}_{3}\right.$ )
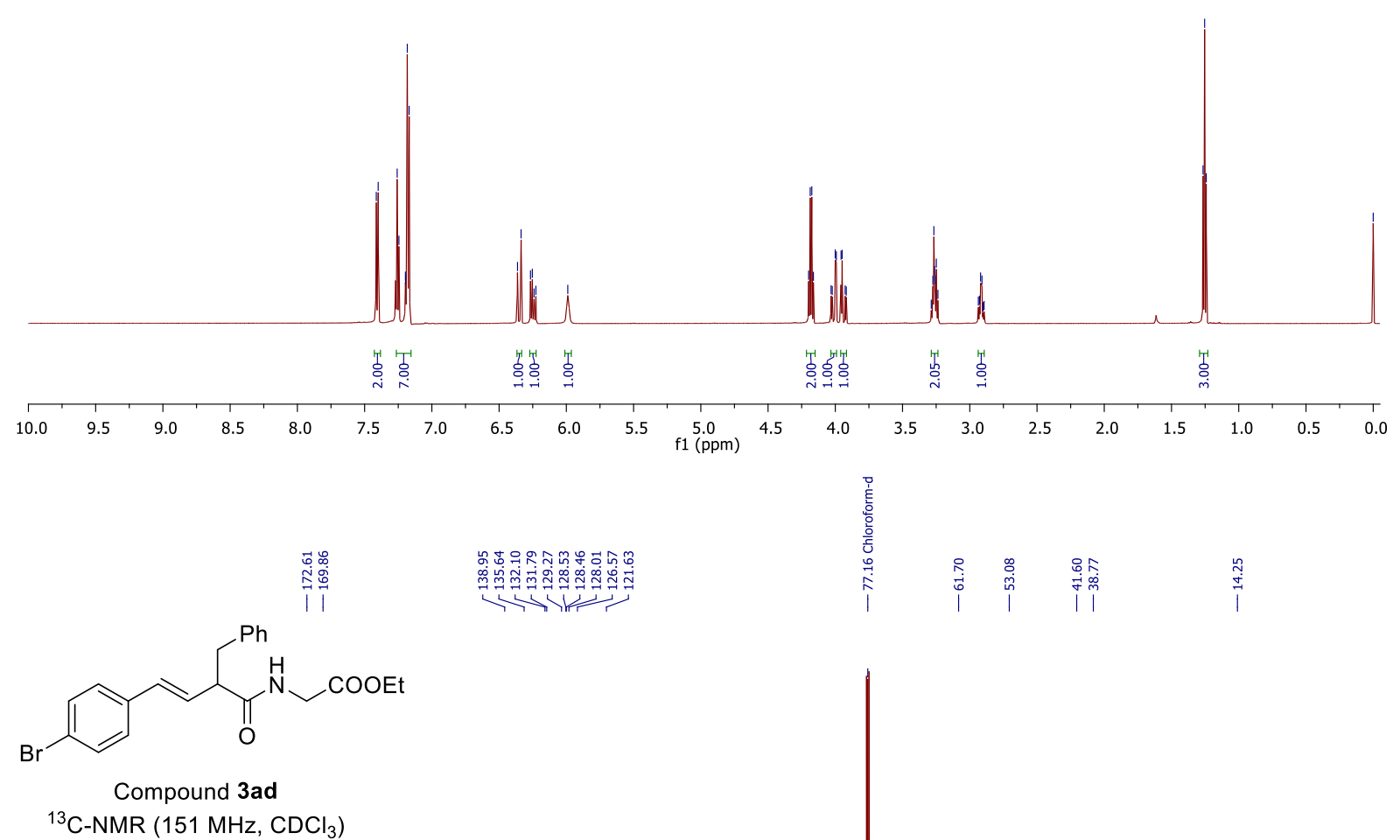

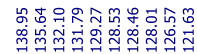

资

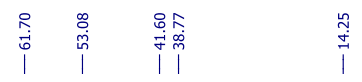

${ }^{13} \mathrm{C}-\mathrm{NMR}\left(151 \mathrm{MHz}, \mathrm{CDCl}_{3}\right.$ )

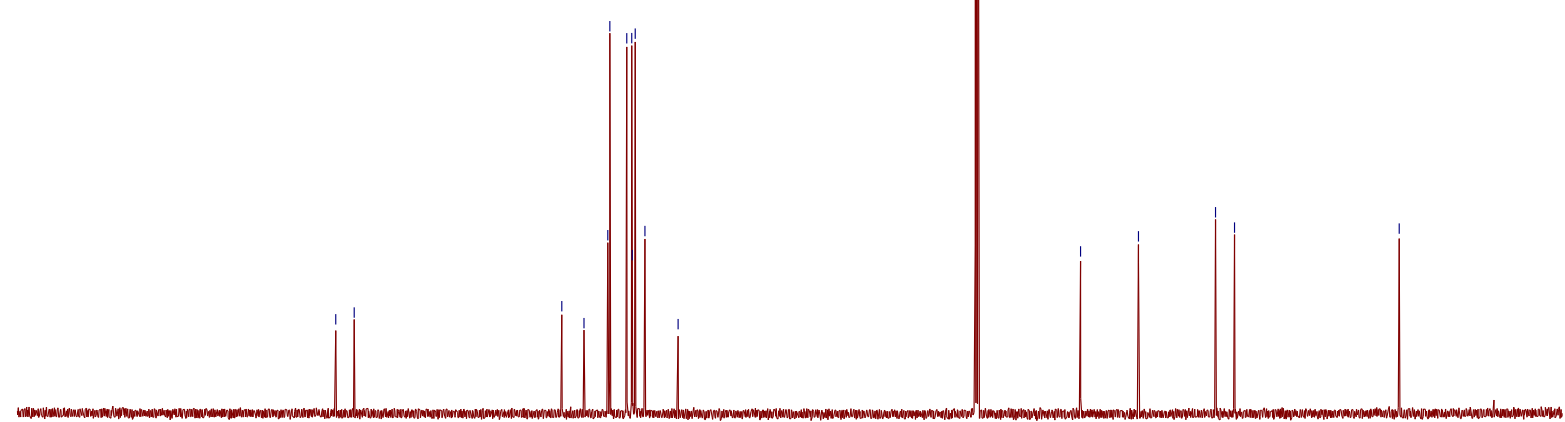

$\begin{array}{llllllllllllllllllllllllllll}220 & 210 & 200 & 190 & 180 & 170 & 160 & 150 & 140 & 130 & 120 & \underset{f}{110}(\mathrm{ppm}) & 100 & 90 & 80 & 70 & 60 & 50 & 40 & 30 & 20 & 10 & 0 & -10\end{array}$ 


\subsection{0. ${ }^{1} \mathrm{H}$ and ${ }^{13} \mathrm{C}$ NMR spectra of 3ae}

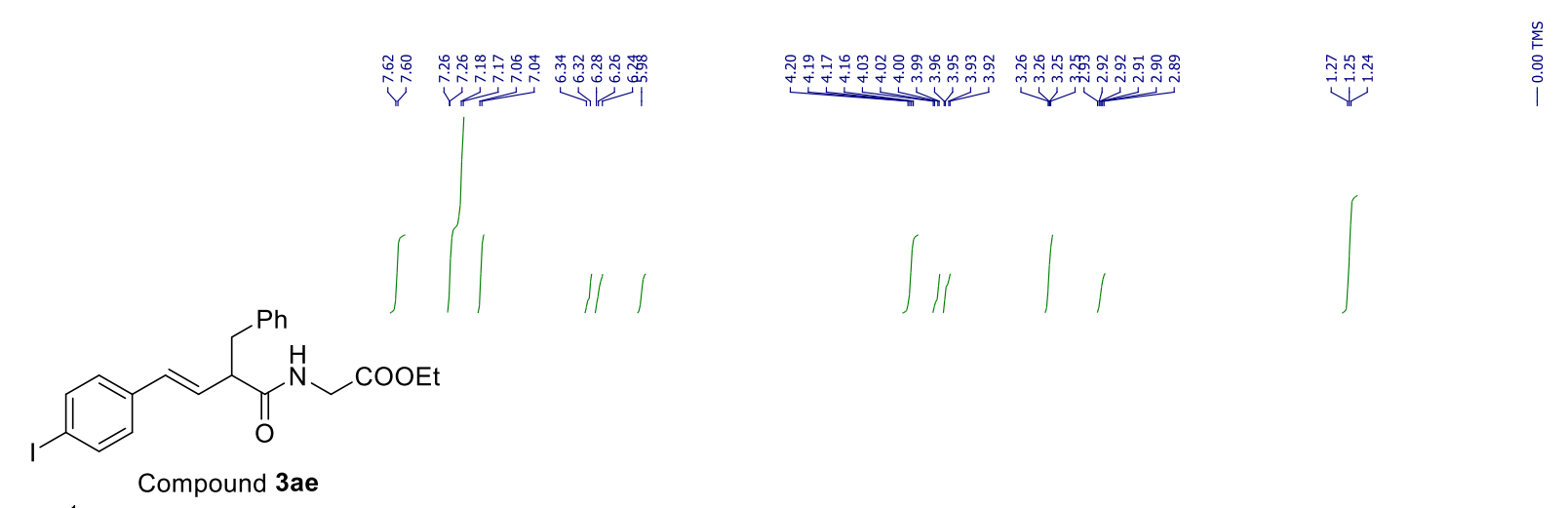

${ }^{1} \mathrm{H}-\mathrm{NMR}\left(600 \mathrm{MHz}, \mathrm{CDCl}_{3}\right)$
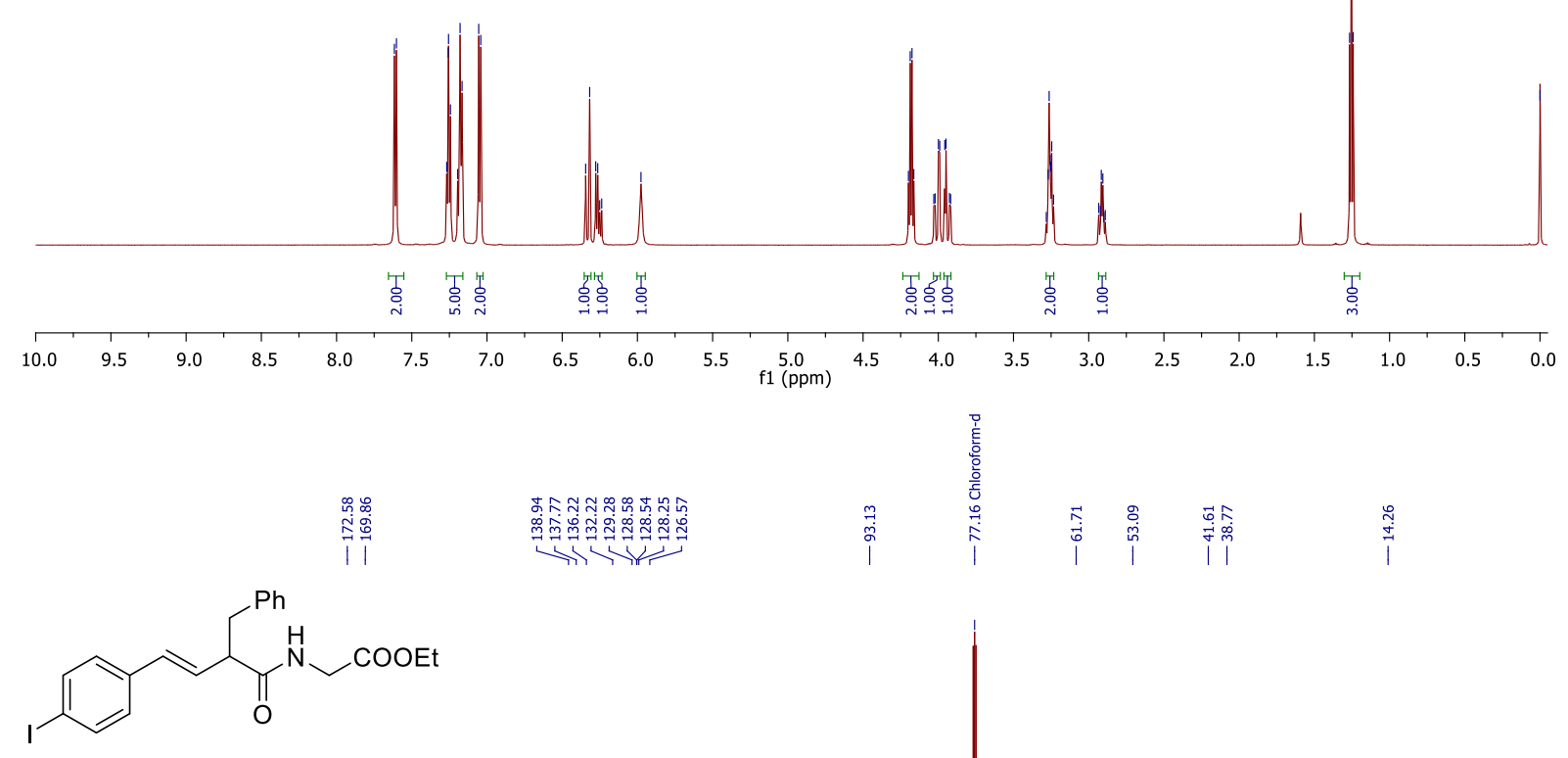

Compound 3ae

${ }^{13} \mathrm{C}-\mathrm{NMR}\left(151 \mathrm{MHz}, \mathrm{CDCl}_{3}\right)$

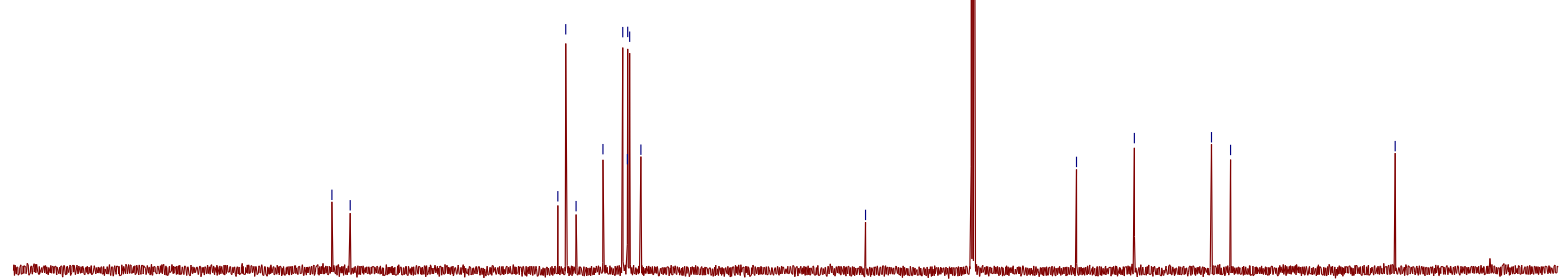

$\begin{array}{lllllllllllllllllllllllllll}220 & 210 & 200 & 190 & 180 & 170 & 160 & 150 & 140 & 130 & 120 & \underset{f 1}{110}(\mathrm{ppm}) & 100 & 90 & 80 & 70 & 60 & 50 & 40 & 30 & 20 & 10 & 0 & -10\end{array}$ 


\subsection{1. ${ }^{1} \mathrm{H}$ and ${ }^{13} \mathrm{C}$ NMR spectra of 3af}
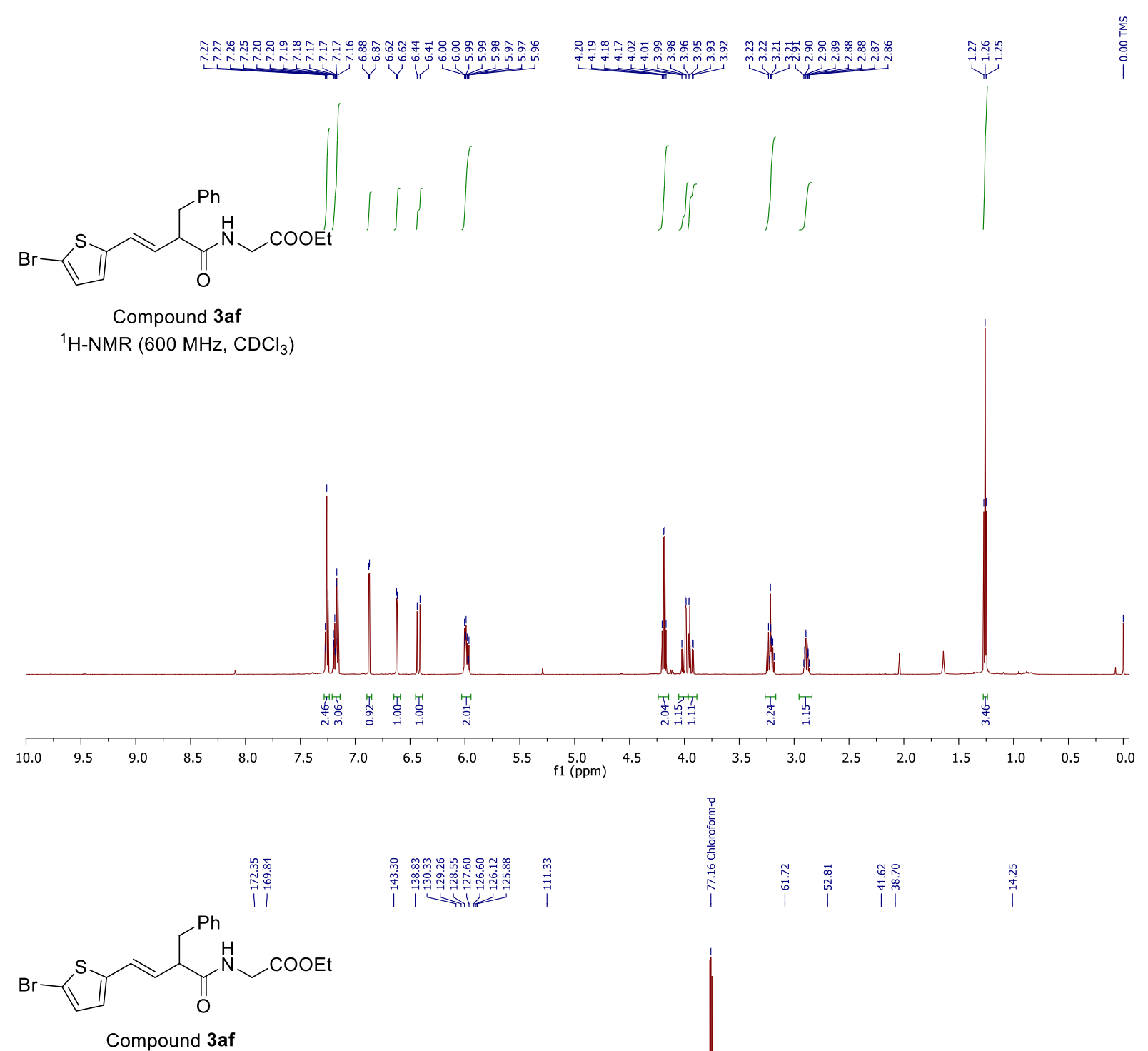

${ }^{13} \mathrm{C}-\mathrm{NMR}\left(151 \mathrm{MHz}, \mathrm{CDCl}_{3}\right)$

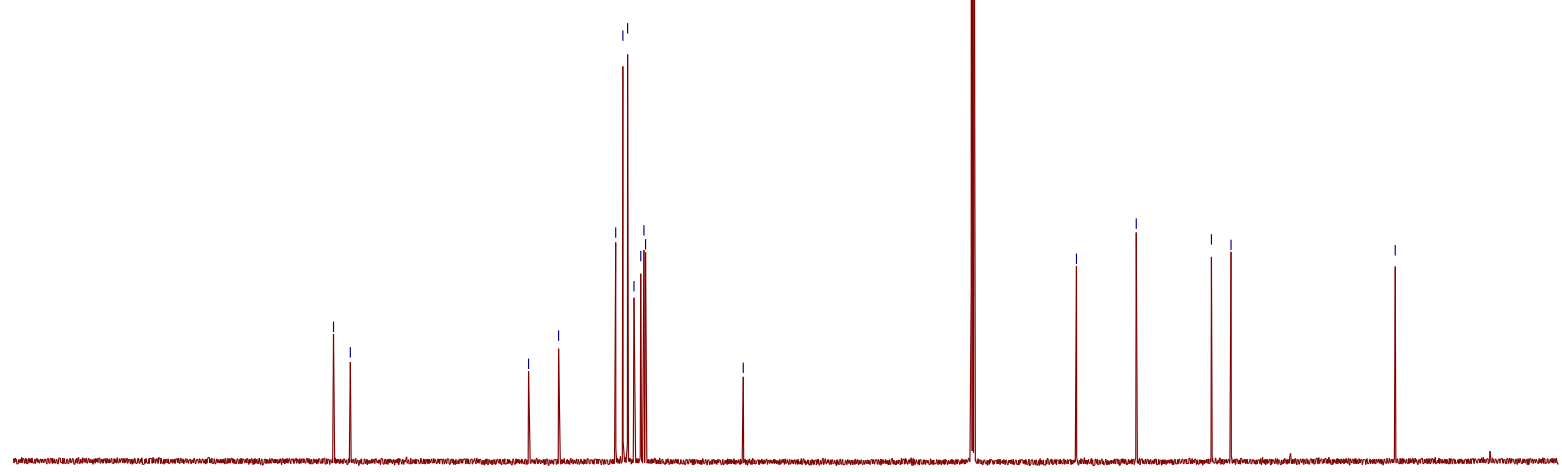

$\begin{array}{llllllllllll}220 & 210 & 200 & 190 & 180 & 170 & 160 & 150 & 140 & 130 & 120 & \begin{array}{c}110 \\ \mathrm{f} 1(\mathrm{ppm})\end{array}\end{array}$ 


\subsection{2. ${ }^{1} \mathrm{H}$ and ${ }^{13} \mathrm{C}$ NMR spectra of $3 a g$}
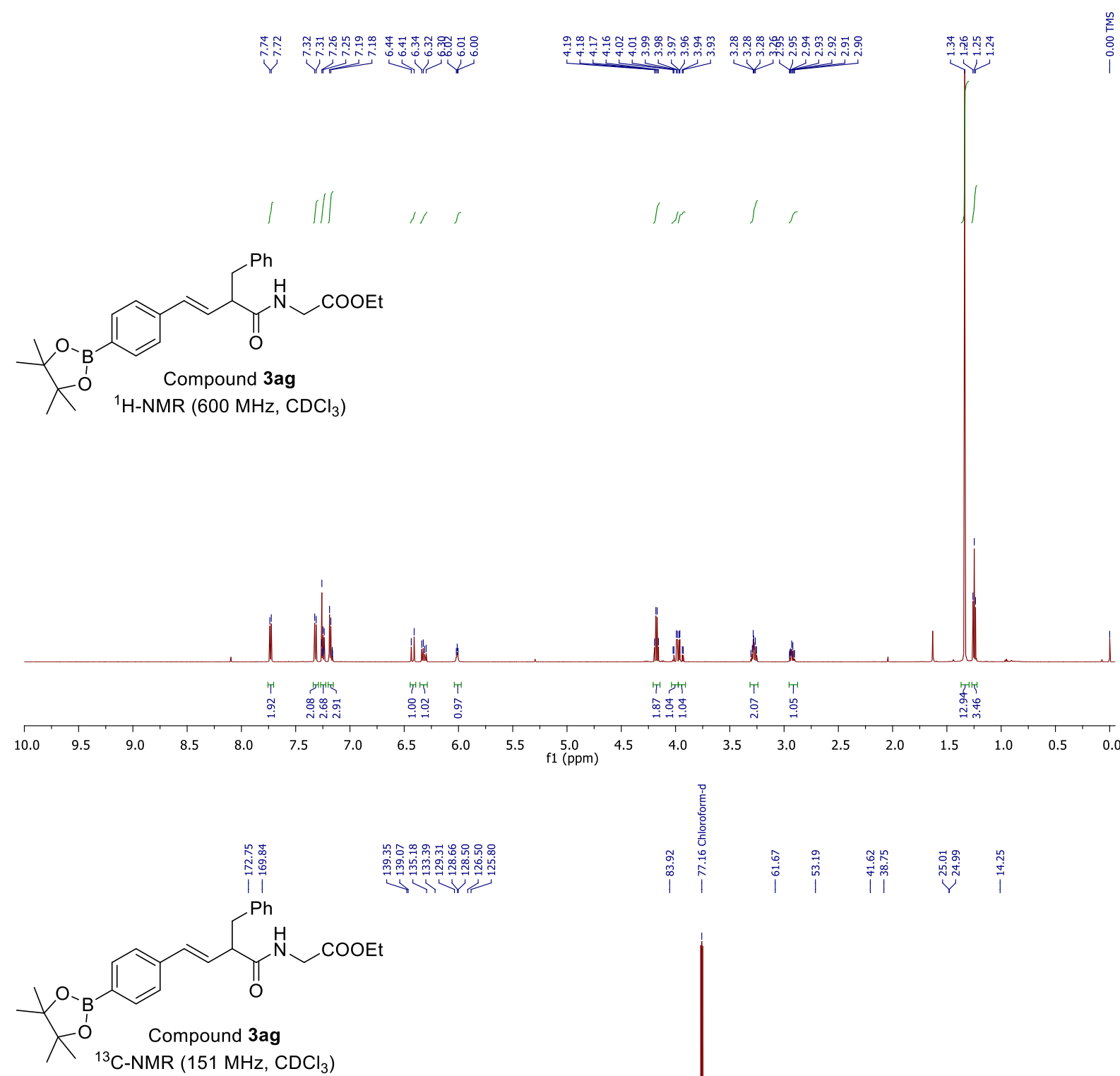

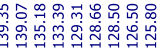

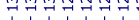




\subsection{3. ${ }^{1} \mathrm{H}$ and ${ }^{13} \mathrm{C}$ NMR spectra of $3 \mathrm{ah}$}

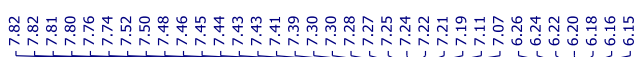

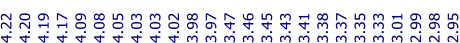
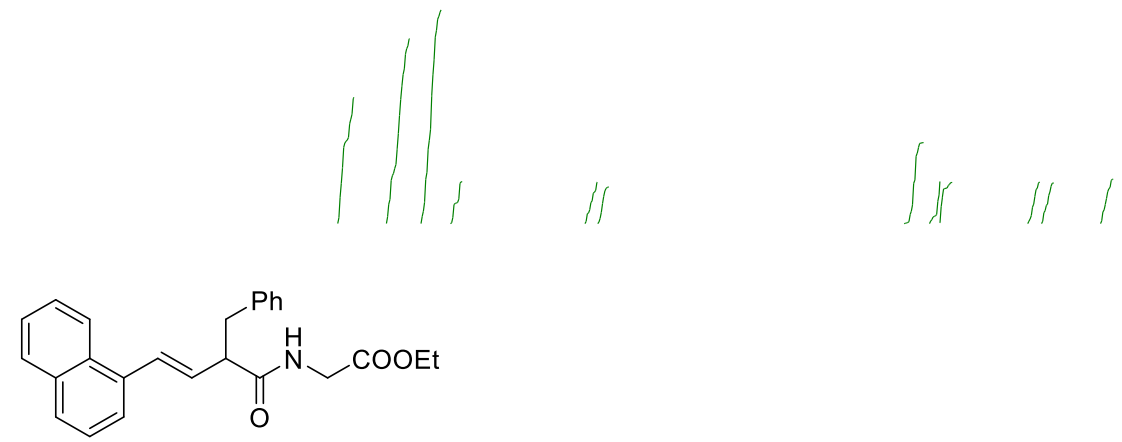

Compound 3ah

${ }^{1} \mathrm{H}-\mathrm{NMR}\left(300 \mathrm{MHz}, \mathrm{CDCl}_{3}\right.$ )

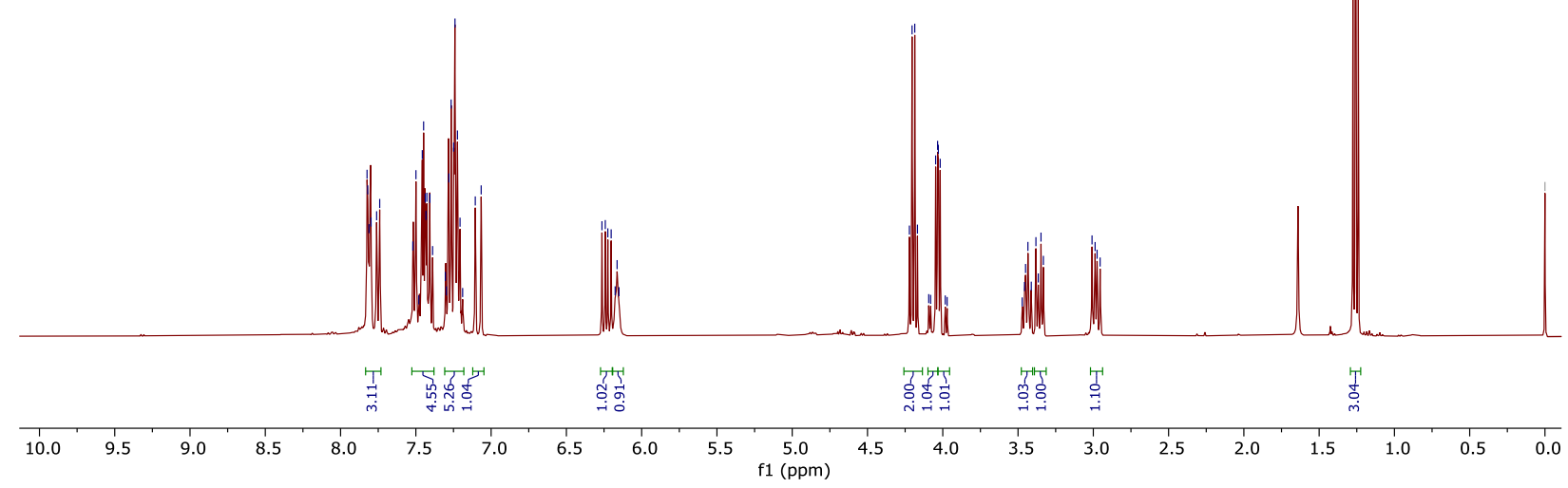

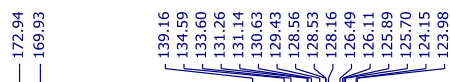

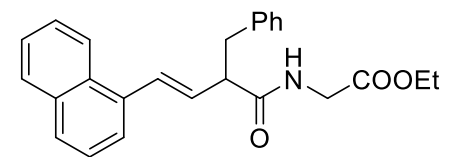

Compound 3 ah

${ }^{13} \mathrm{C}-\mathrm{NMR}\left(75 \mathrm{MHz}, \mathrm{CDCl}_{3}\right)$

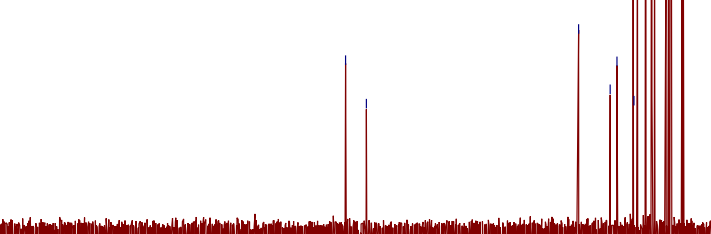

$\begin{array}{lllllllllllll}220 & 210 & 200 & 190 & 180 & 170 & 160 & 150 & 140 & 130 & 120 & 110 & 100 \\ & & & & & & & & & & \end{array}$ 


\subsection{4. ${ }^{1} \mathrm{H}$ and ${ }^{13} \mathrm{C}$ NMR spectra of 3ai}
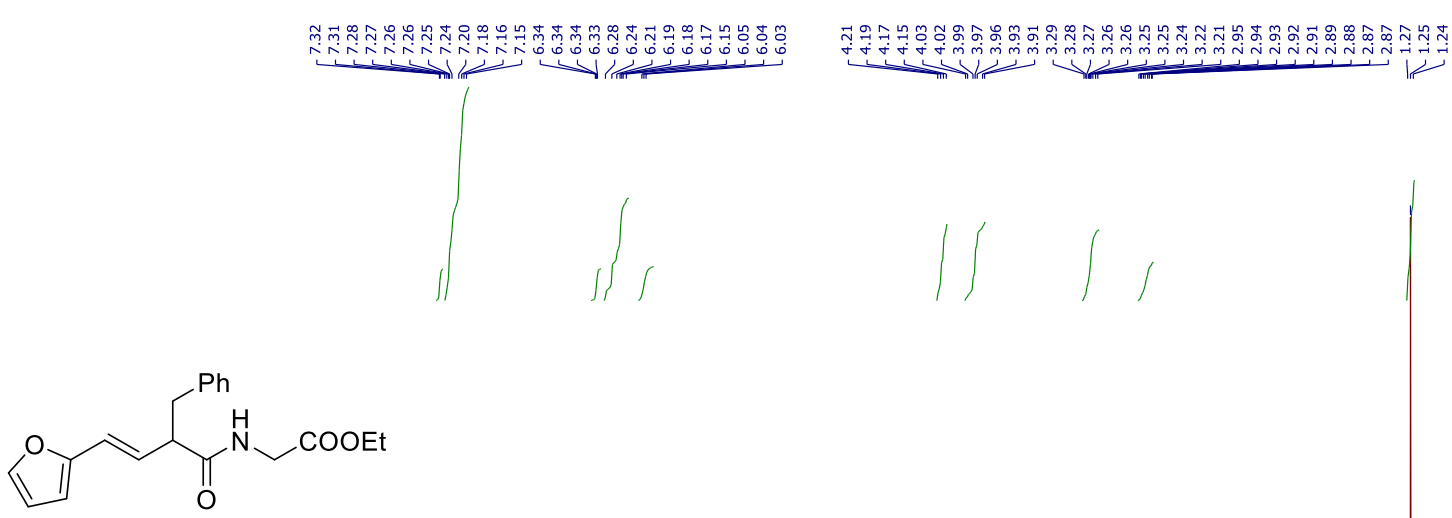

Compound 3ai

${ }^{1} \mathrm{H}-\mathrm{NMR}\left(300 \mathrm{MHz}, \mathrm{CDCl}_{3}\right.$ )

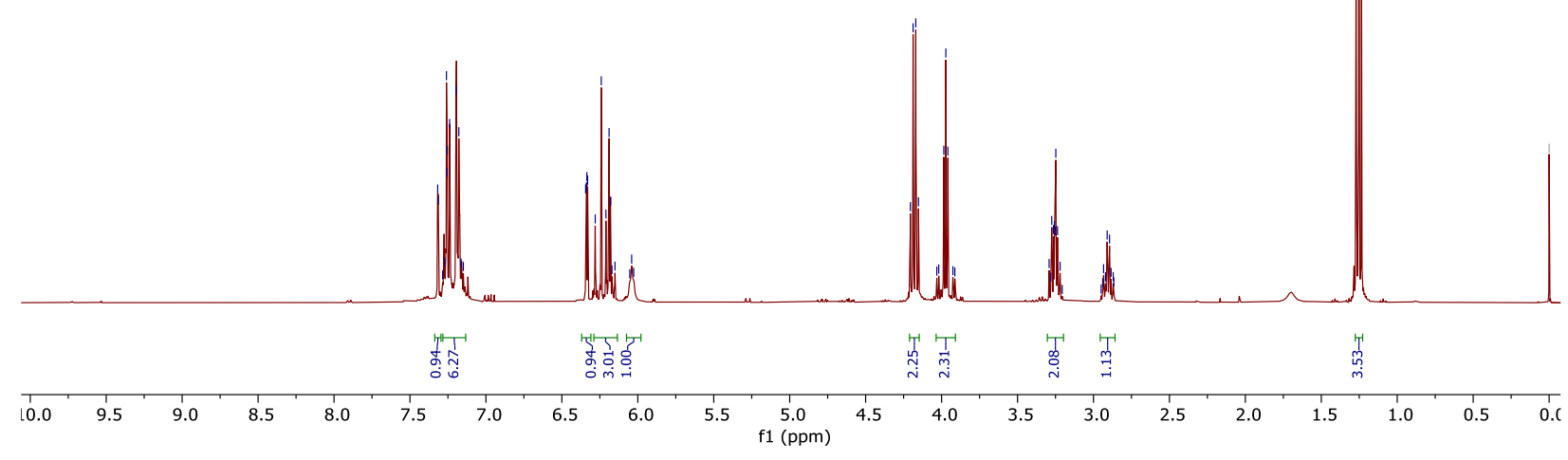

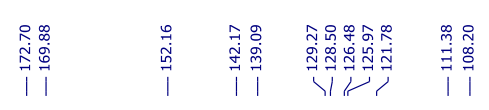

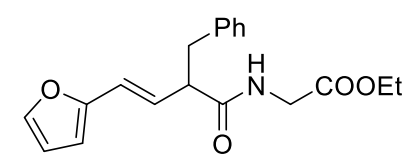

Compound 3ai

${ }^{13} \mathrm{C}-\mathrm{NMR}\left(75 \mathrm{MHz}, \mathrm{CDCl}_{3}\right.$ )

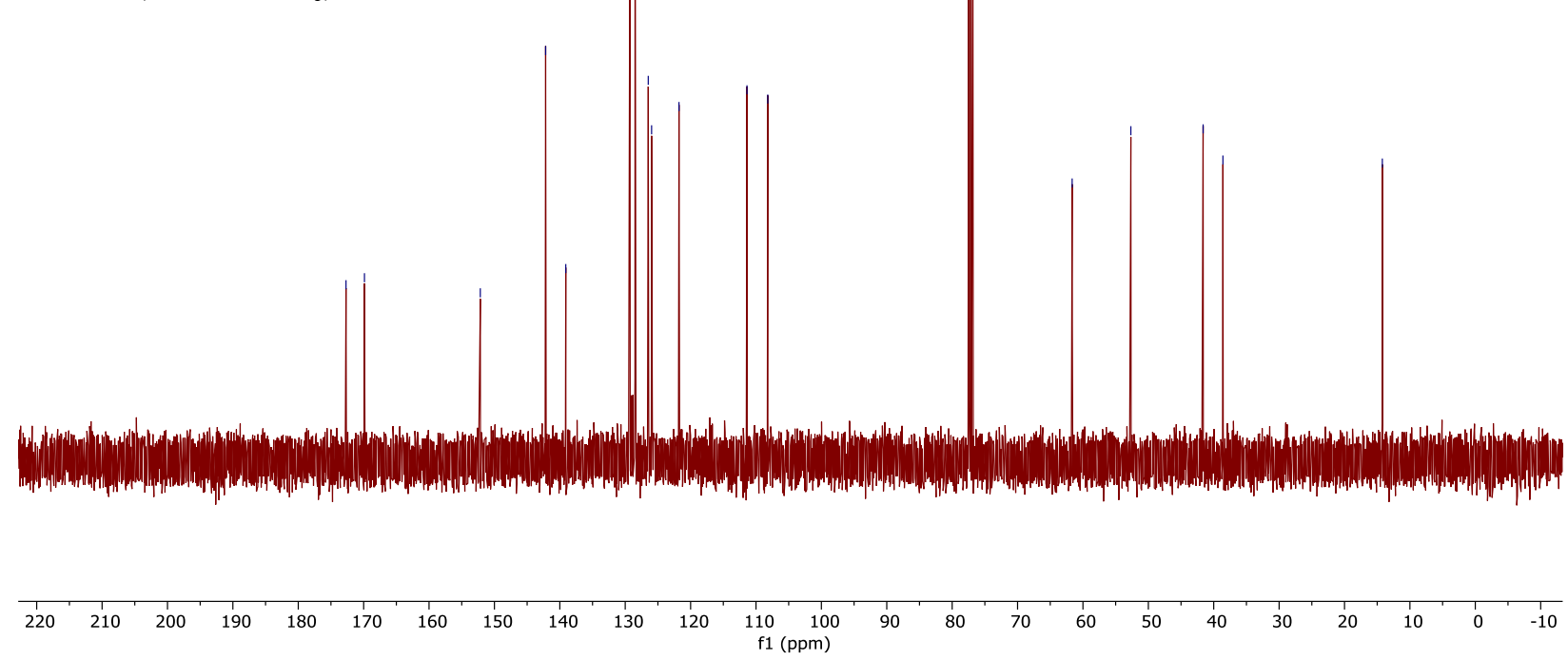




\subsection{5. ${ }^{1} \mathrm{H}$ and ${ }^{13} \mathrm{C}$ NMR spectra of $3 a j$}
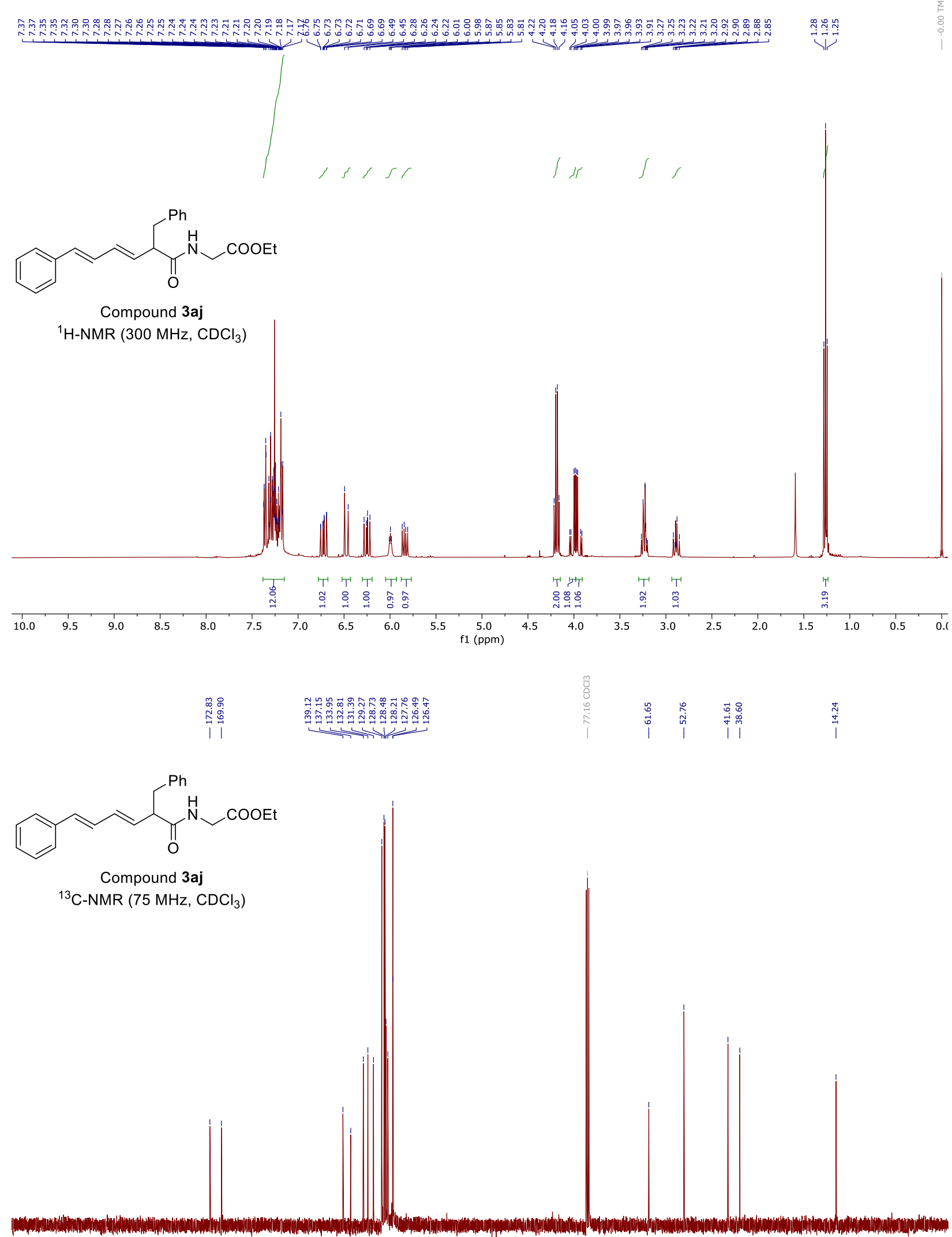

$\begin{array}{llllllllllllllllllllllllll}220 & 210 & 200 & 190 & 180 & 170 & 160 & 150 & 140 & 130 & 120 & 110 & 100 & 90 & 80 & 70 & 60 & 50 & 40 & 30 & 20 & 10 & 0 & -10\end{array}$ 
4.36. ${ }^{1} \mathrm{H}$ and ${ }^{13} \mathrm{C}$ NMR spectra of 3an
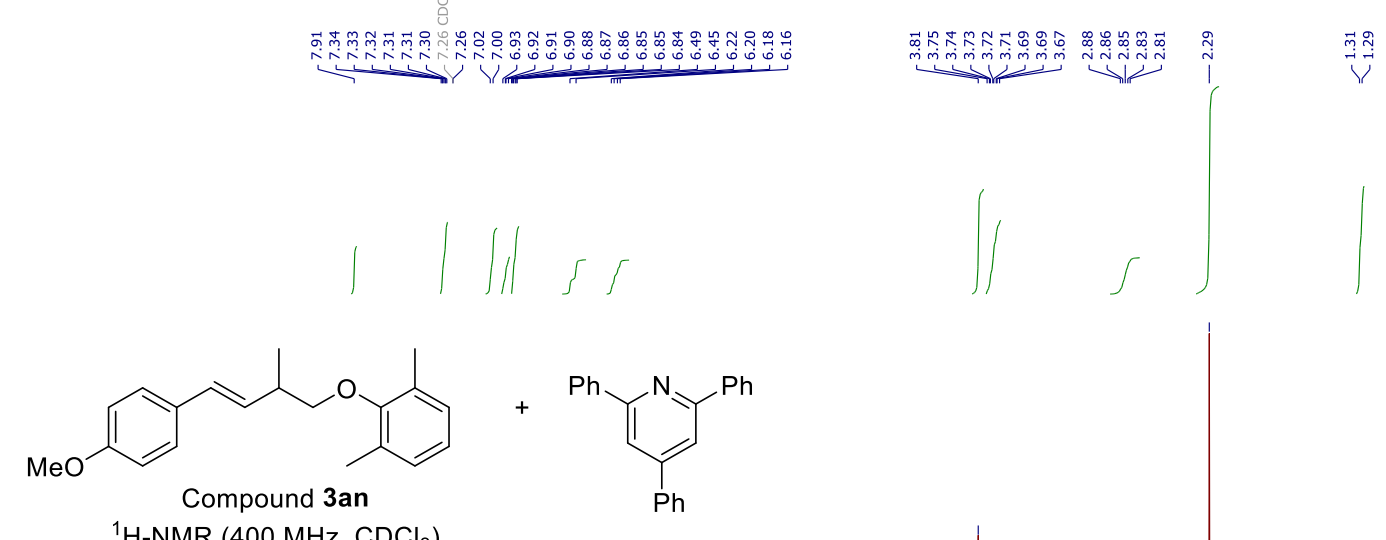

${ }^{1} \mathrm{H}-\mathrm{NMR}\left(400 \mathrm{MHz}, \mathrm{CDCl}_{3}\right)$

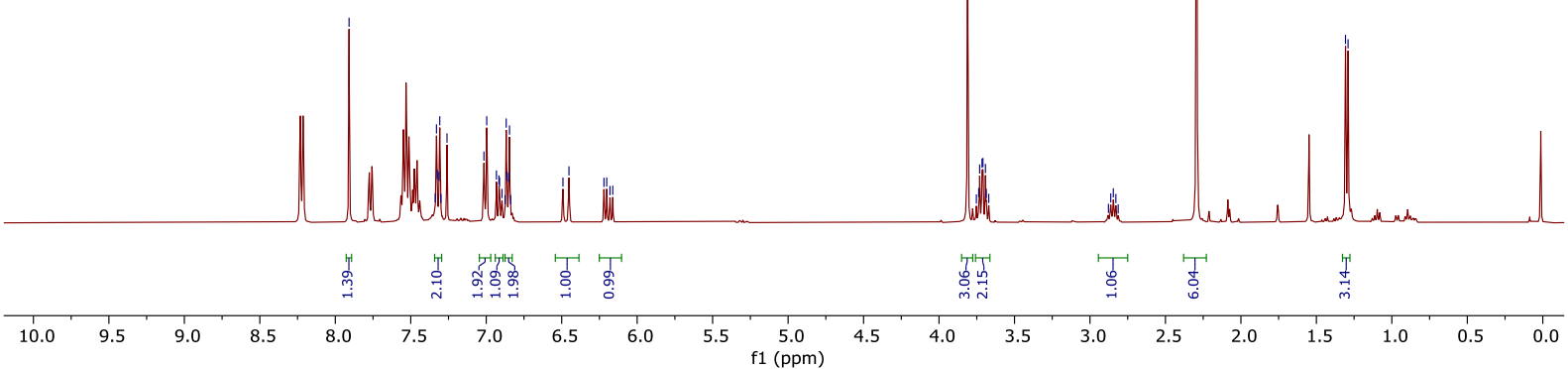

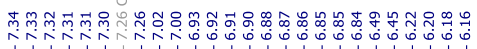

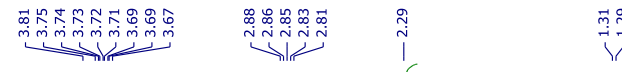

$\iint S s$
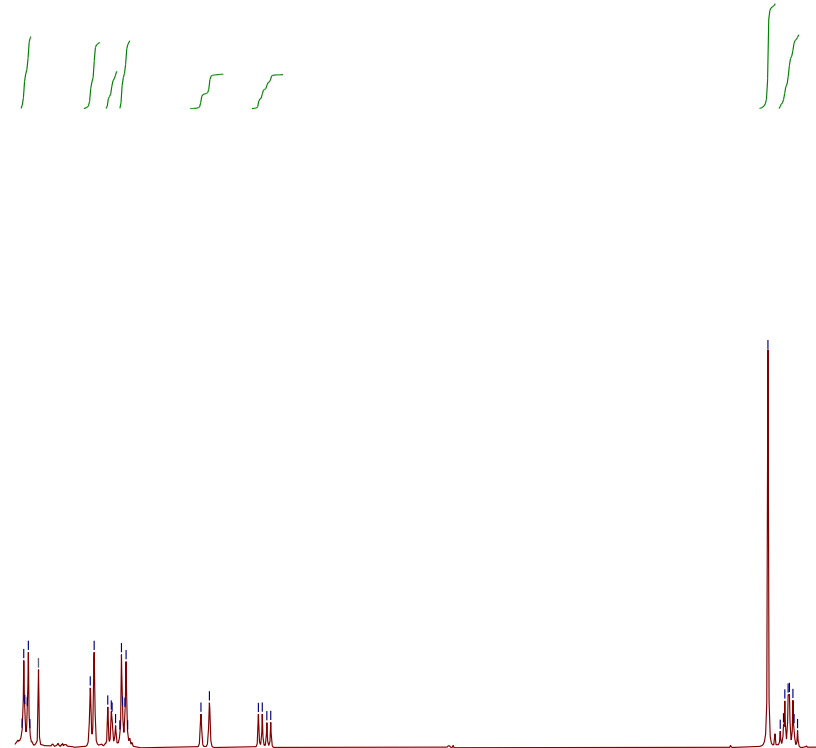

국

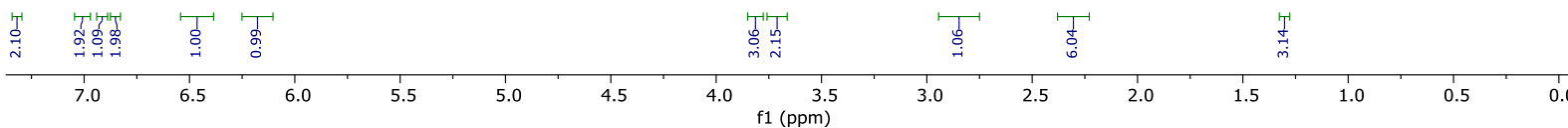



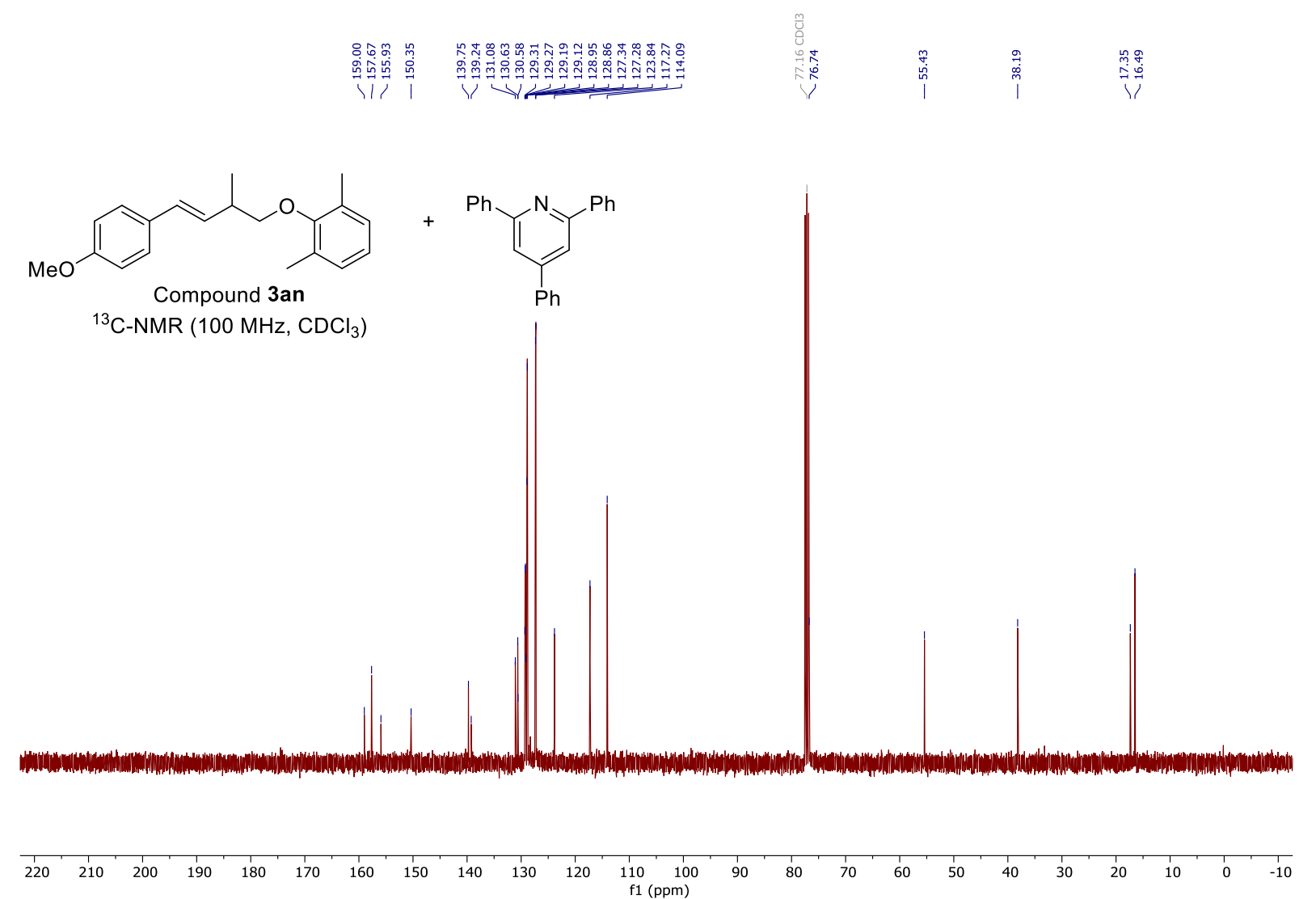